US Army Corps

of Engineers ${ }_{\circledast}$

Engineer Research and

Development Center

Geospatial Research and Engineering

\title{
Signal Propagation Modeling in Complex, Three-Dimensional Environments
}

D. Keith Wilson, Daniel J. Breton, Lauren E. Waldrop, Danney R. Glaser, $\quad$ April 2021 Ross E. Alter, Carl R. Hart, Wesley M. Barnes, Michael T. Ekegren, Michael B. Muhlestein, Manisha Mishra, M. Andrew Niccolai, Michael J. White, Christian Borden, and Ethan Fahy
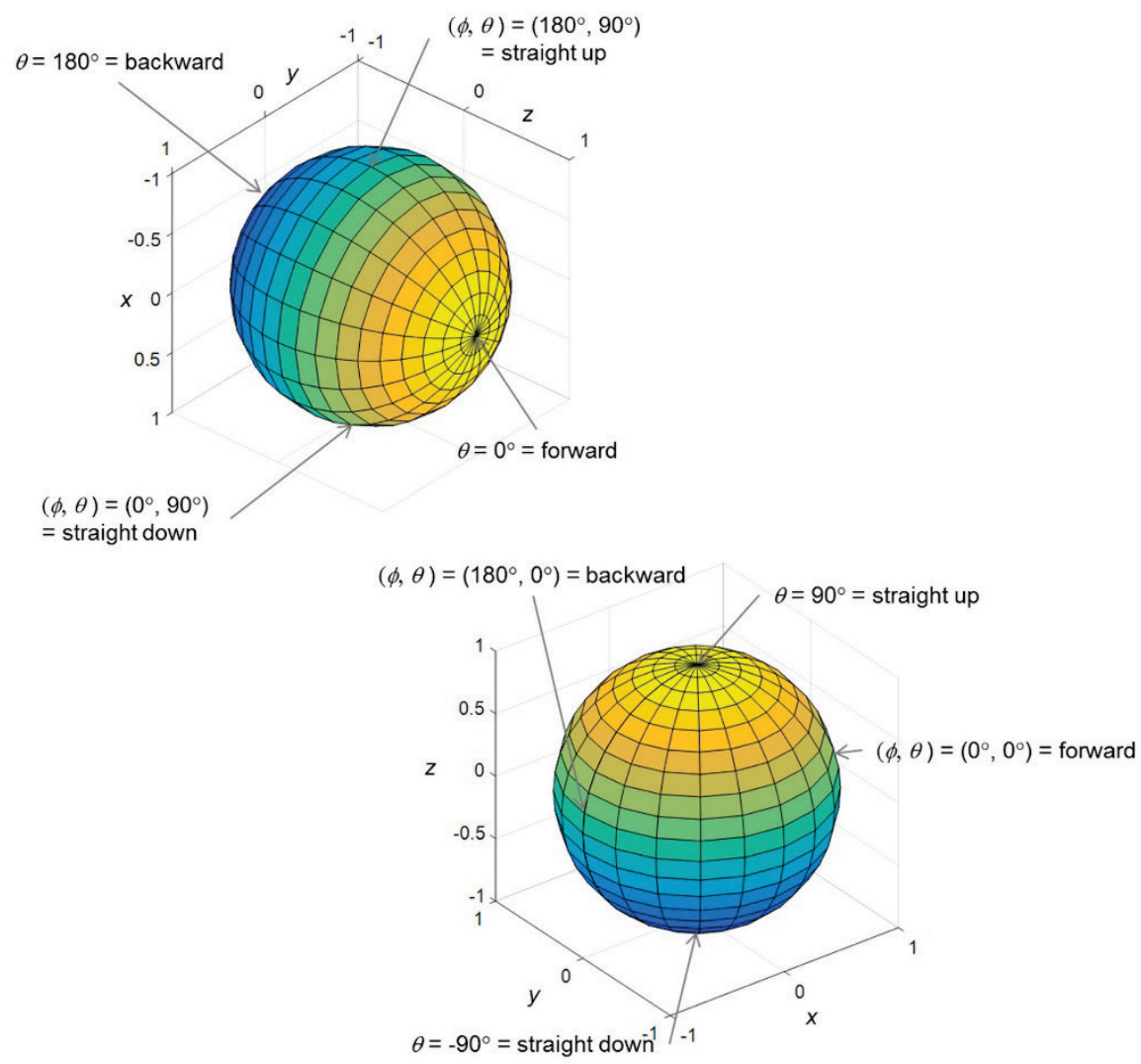
The U.S. Army Engineer Research and Development Center (ERDC) solves the nation's toughest engineering and environmental challenges. ERDC develops innovative solutions in civil and military engineering, geospatial sciences, water resources, and environmental sciences for the Army, the Department of Defense, civilian agencies, and our nation's public good. Find out more at www.erdc.usace.army.mil.

To search for other technical reports published by ERDC, visit the ERDC online library at https://erdclibrary.on.worldcat.org/discovery. 


\section{Signal Propagation Modeling in Complex, Three-Dimensional Environments}

D. Keith Wilson, Daniel J. Breton, Lauren E. Waldrop, Danney R. Glaser, Ross E. Alter, Carl R. Hart, Wesley M. Barnes, Michael T. Ekegren, Michael B. Muhlestein, Manisha Mishra, and M. Andrew Niccolai

Cold Regions Research and Engineering Laboratory (CRREL)

U.S. Army Engineer Research and Development Center (ERDC)

72 Lyme Road

Hanover, NH 03755-1290

Michael J. White

Construction Engineering Research Laboratory (CERL)

U.S. Army Engineer Research and Development Center (ERDC)

2902 Newmark Drive

Champaign, IL 61822

Christian Borden and Ethan Fahy

Atmospheric and Environmental Research, Inc.

131 Hartwell Avenue \#4

Lexington, MA 02421

Technical Report (TR)

Approved for public release; distribution unlimited.

Prepared for Headquarters, U.S. Army Corps of Engineers

Washington, DC 20314-1000

Under ERDC 6.2 Geospatial Research and Engineering (GRE) Army Terrestrial- Environmental Modeling and Intelligence System Science Technology Objective-Research (ARTEMIS STO-R) under Work Item ARTEMIS CRREL T42 053HJO, Funding Account Number U4357509 


\section{Abstract}

The Signal Physics Representation in Uncertain and Complex Environments (SPRUCE) work unit, part of the U.S. Army Engineer Research and Development Center (ERDC) Army Terrestrial-Environmental Modeling and Intelligence System (ARTEMIS) work package, focused on the creation of a suite of three-dimensional (3D) signal and sensor performance modeling capabilities that realistically capture propagation physics in urban, mountainous, forested, and other complex terrain environments. This report describes many of the developed technical capabilities. Particular highlights are (1) creation of a Java environmental data abstraction layer for $3 \mathrm{D}$ representation of the atmosphere and inhomogeneous terrain that ingests data from many common weather forecast models and terrain data formats, (2) extensions to the Environmental Awareness for Sensor and Emitter Employment (EASEE) software to enable 3D signal propagation modeling, (3) modeling of transmitter and receiver directivity functions in 3D including rotations of the transmitter and receiver platforms, (4) an Extensible Markup Language/JavaScript Object Notation (XML/JSON) interface to facilitate deployment of web services, (5) signal feature definitions and other support for infrasound modeling and for radio-frequency (RF) modeling in the very high frequency (VHF), ultra-high frequency (UHF), and super-high frequency (SHF) frequency ranges, and (6) probabilistic calculations for line-of-sight in complex terrain and vegetation.

DISCLAIMER: The contents of this report are not to be used for advertising, publication, or promotional purposes. Citation of trade names does not constitute an official endorsement or approval of the use of such commercial products. All product names and trademarks cited are the property of their respective owners. The findings of this report are not to be construed as an official Department of the Army position unless so designated by other authorized documents.

DESTROY THIS REPORT WHEN NO LONGER NEEDED. DO NOT RETURN IT TO THE ORIGINATOR. 


\section{Contents}

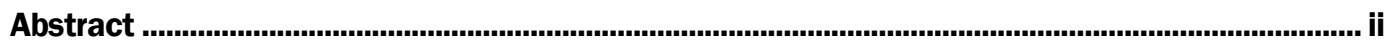

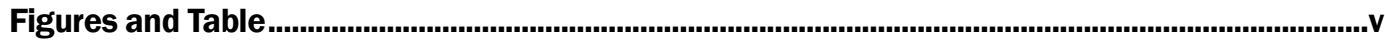

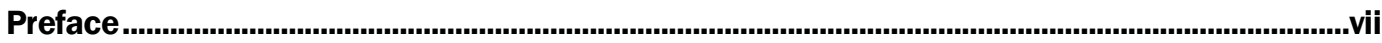

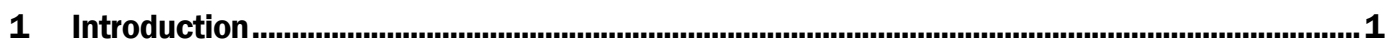

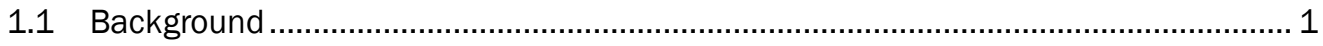

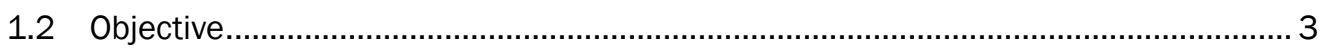

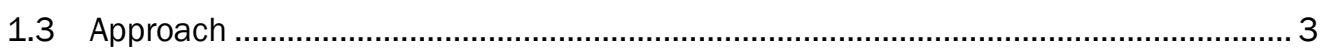

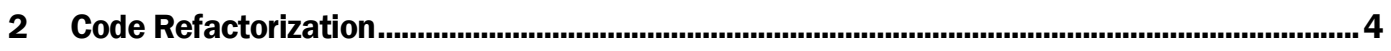

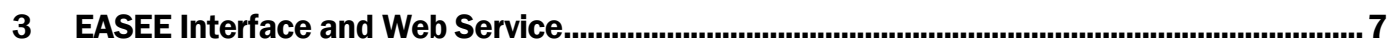

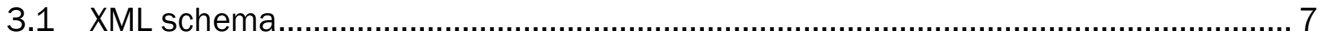

3.2 Interfacing with the ArcGIS Desktop ................................................................. 8

3.3 EASEE web services.............................................................................. 12

3.4 Simple Object Access Protocol (SOAP) and Representational State Transfer (RESTful) endpoints ...................................................................................... 13

4 Platform Enhancements...................................................................................................16

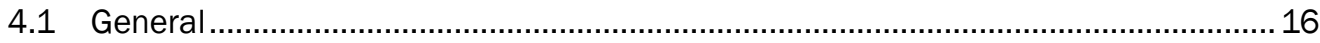

4.2 Dynamic loading of platform signatures........................................................... 17

4.3 Source and receiver directivity ......................................................................... 19

4.4 Coordinate rotations ........................................................................................ 22

4.5 Human auditory modeling ................................................................................... 23

4.6 New platforms and enhancements ........................................................... 24

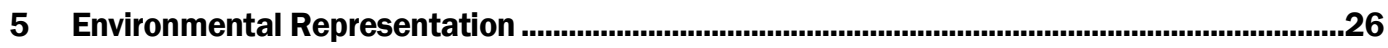

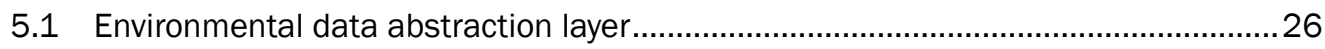

5.2 Geographic coordinate transformations.................................................................. 28

5.3 Terrain representation and inference of terrain properties......................................30

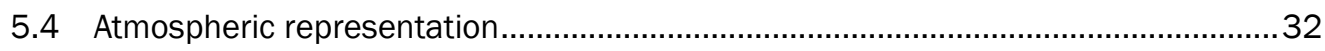

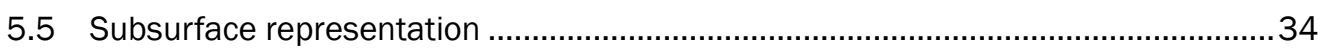

6 Signal Propagation and Noise Modeling...............................................................................35

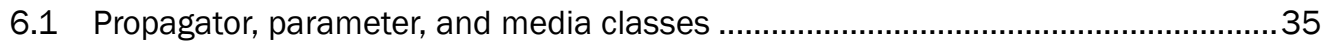

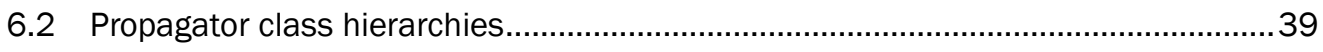

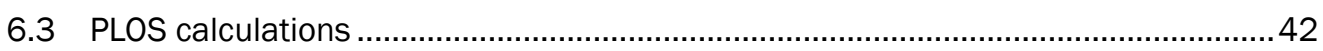

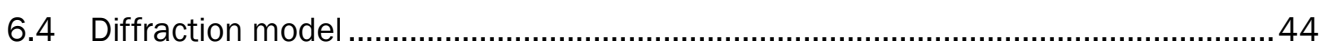

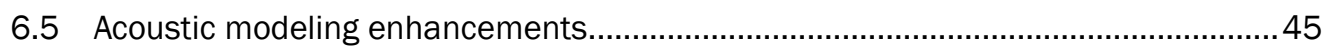

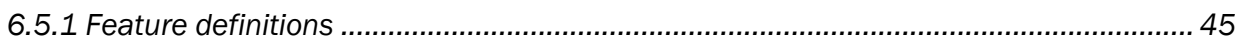

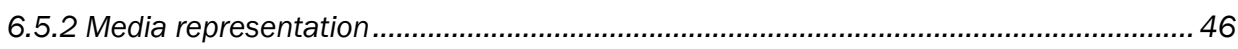

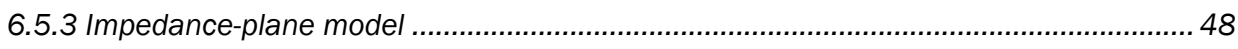

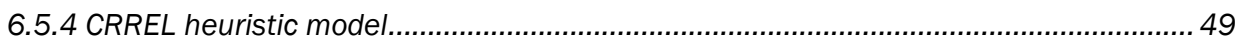




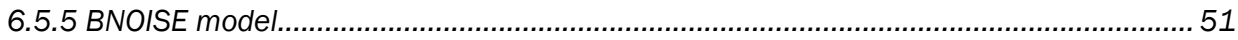

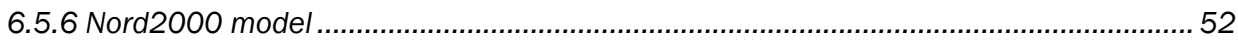

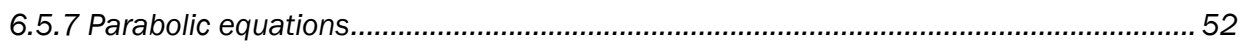

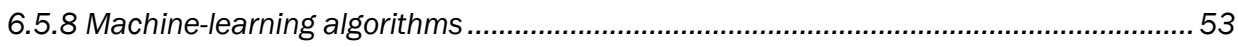

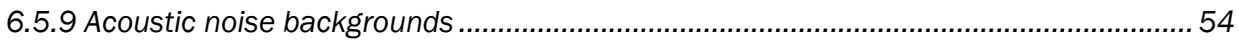

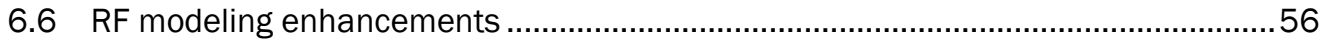

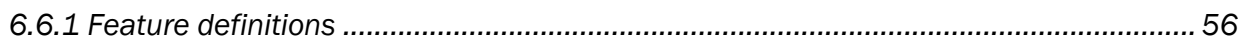

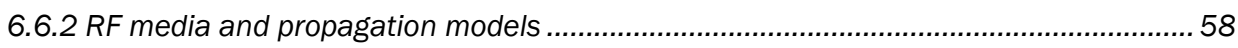

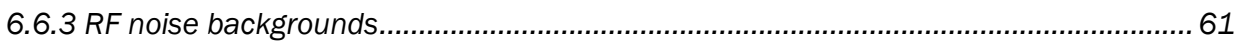

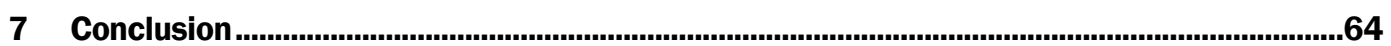

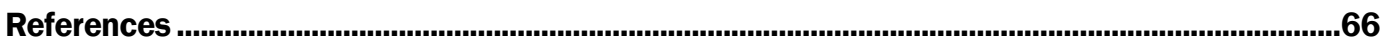

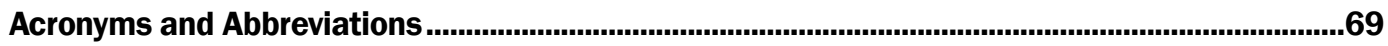

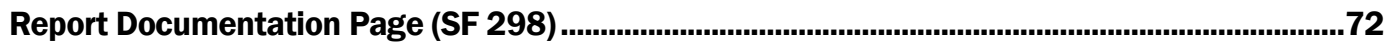




\section{Figures and Table}

\section{Figures}

1 Stages in the EASEE modeling framework. The individual stages (emission, propagation, sensing, processing, and fusion) are defined through Java interfaces, which encapsulate modeling capabilities for acoustic, seismic, RF, infrared, visible, and chemical/biological signals. Java generics are used to ensure that only compatible models are interfaced together

2 Conceptual diagram of EASEE's software components. EASEELib encapsulates KNEE (a stripped-down version of the EASEE software) and houses many advanced modules that provide enhanced capabilities (purple boxes). EASEELib is also able to facilitate interaction with a host of interfaces (orange boxes).

3 EASEE ArcGIS toolbar, which integrates with ArcMap and allows users to select specific configuration settings for an EASEE calculation

$4 \quad$ Input form pop-up triggered by the Landcover and Soil button on the EASEE toolbar. This is just one example of an input form. Others are available for selecting signal modalities and fidelities, terrain, atmospheric conditions and background noise, and fixed and variable platform attributes. 9

5 Demonstration of the general architecture and flow of communication between EASEE and the ArcGIS Desktop application

6 Output corresponding to a probability of detection calculation run in EASEE. The green areas are indicative of a high probability of detection of the helicopter (annotated as a blue diamond) by a hostile human listener on the ground, while the red is indicative of a low probability of detection

7 Input data interfaces for the EASEE web service

8 Output product interfaces for the EASEE web service.

9 Relationships between classes associated with platforms in EASEE. This diagram provides a simplified view of the essential fields and methods available within each class and the PlatformTyping interface. The focus is primarily on the relationships between component parts as opposed to the contents of each component.

10 Inheritance tree for the EASEE representations (Java classes) for directivity functions. This functionality may be used for both source radiation and sensor beam patterns. The classes shown with a white font are abstract; the black font indicates a non-abstract class

11 Spherical angle systems used to describe directivity functions in Acoustic Propagation and Emulation Toolset (APET) (left) and EASEE (right)

12 Hierarchy of the environmental scenarios in EASEE and the objects contained within each scenario. Blue font indicates additions made as part of the SPRUCE project

13 Flowchart for inferring unspecified terrain properties from the specified ones .31

14 The EASEE environmental abstraction layer (EnvironScenario and its subclasses) is constructed from a variety of data resources for terrain elevation, landcover, soils, and atmospheric forecasts and models. The environmental data are converted to the propagator media, which are then used for signal propagation modeling 
15 Process by which propagation calculations are run in EASEE. The propagator (computational) parameters are constructed and then used to construct the propagator, which encapsulates the actual signal propagation model. The environmental representation is used to construct the propagator media. The media, calculation grid, and signature are dynamically sent to the propagator, which performs its task and sends the output to the sensor model

16 Left: Parent class for the propagators (BasePropagator) and its direct subclasses. Right: Parent class for the structured-grid propagators (BaseStructGridPropagator) and its direct subclasses.

17 Left: Base class for propagator parameters (BasePropagatorParams) and some of its subclasses. Right: Base class for propagator media (BasePropagatorMedia) and some of its subclasses.

18 Geometry of the PLOS calculation. Here $\boldsymbol{d a}$ is the path length in the air layer, $\boldsymbol{d} \boldsymbol{c}$ is the path length in the canopy layer, and $\boldsymbol{d} \boldsymbol{s}$ is the path length in the subcanopy layer

19 Acoustic and infrasonic feature classes in EASEE. There are three broad feature sets (families), which are coded as Java interfaces: AcousticSpectralFeature ( $6.3 \mathrm{~Hz}$ to $20 \mathrm{kHz}$ ), AudibleSpectralFeature (20 Hz to $20 \mathrm{kHz}$ ), and InfrasonicSpectralFeature (1 Hz to $16 \mathrm{~Hz})$. Within each of these families, Java enumeration classes define specific features

20 Acoustic media classes in EASEE. These classes represent the acoustical properties of the environment, as appropriate to signal propagation models with varying characteristics.

21 Acoustic propagators (propagation algorithms) implemented in EASEE, as grouped according to the medium representation upon which they are based

24 RF feature classes in EASEE. Several feature sets (families) are defined through Java interfaces. Two of these, namely RFCentiMeterSpectralFeature (for the SHF/EHF frequency range) and RFMeterSpectralFeature (for the VHF/UHF frequency range) have been developed extensively, including Java enumeration classes to define specific features

25 RF propagators (propagation algorithms) implemented in EASEE, as grouped according to the medium representation upon which they are based

26 Plots of $\mathbf{F a m}$ as a function of frequency for (a) the standard ITU + Hagn \& Sailors (1979), and (b) the CRREL 2014 noise model, based on the work of Wagstaff and Merricks (2005). The change in slope at $200 \mathrm{MHz}$ results from the changeover from VHF to UHF noise constants

\section{Table}

1 Constants for the ITU standard and CRREL 2014 manmade noise models. VHF constants valid between 30 and 200 MHz; UHF constants valid between 200 and $1000 \mathrm{MHz}$ 


\section{Preface}

This study was conducted for the U.S. Army Corps of Engineers. Funding was provided by the Engineer Research and Development Center (ERDC) Geospatial Research and Engineering (GRE) program Army TerrestrialEnvironmental Modeling and Intelligence System Science Technology Objective-Research (ARTEMIS STO-R) under Work Item ARTEMIS CRREL T42 053HJo, Funding Account Number U4357509. The GRE technical monitor was Dr. Robert Davis, U.S. Army Engineer Research and Development Center, Cold Regions Research and Engineering (ERDC-CRREL).

The work was performed by the Signature Physics Branch, CEERD-RRD, of the Research \& Engineering Division, CEERD-RR, Engineer Research and Development Center, Cold Regions Research and Engineering Laboratory (ERDC-CRREL). At the time of publication, Dr. M. Andrew Niccolai was Chief, CEERD-RRD and Dr. George Calfas was Acting Chief, CEERDRR. The Deputy Director of ERDC-CRREL was Mr. David B. Ringelberg and the Director was Dr. Joseph L. Corriveau.

COL Teresa A. Schlosser was Commander of ERDC, and Dr. David W. Pittman was the Director. 
THIS PAGE INTENTIONALLY LEFT BLANK 


\section{Introduction}

\subsection{Background}

Characterization of environmental effects on signal detectability and sensor performance is important to the planning and execution of a great variety of military and security missions, including routing of aircraft to minimize audibility and visibility during maneuver and covert operations, laying out sensor networks for detection and tracking of hostile forces, and selection of surveillance assets for air defense and facility protection. Efficient and versatile modeling and simulation capabilities are needed to effectively support these diverse scenarios of interest. These capabilities must be packaged into interfaces that are conveniently accessible to soldiers, intelligence analysts, engineers, and other users, who are not necessarily experts in signal phenomenology. Trade-offs between model accuracy and speed must be appropriately balanced to the application.

The Environmental Awareness for Sensor and Emitter Employment (EASEE) software was developed to address these needs. EASEE is government-owned, off-the-shelf (GOTS) software that runs on any operating system. One of EASEE's main attractions is that it supports realistic, physicsbased modeling of signal emission, transmission, and sensor performance for all types of signal modalities. EASEE can use atmospheric and terrain data from many sources and can be integrated into a wide variety of user interfaces. Currently, acoustic, infrasonic, seismic, radio frequency (RF), visible, infrared, and chem-bio calculations have been implemented.

Figure 1 shows the basic framework of EASEE. Innovative technical approaches were incorporated into many aspects of the software design, including an object-oriented description of signal statistics, inferences, platforms, and modeling capabilities; algorithms for optimizing selection and placement of sensors; and interfaces for integration into geographic information systems (GISs) and web services. The result is a highly flexible framework for integrating realistic physics-based modeling capabilities. 
Figure 1. Stages in the EASEE modeling framework. The individual stages (emission, propagation, sensing, processing, and fusion) are defined through Java interfaces, which encapsulate modeling capabilities for acoustic, seismic, RF, infrared, visible, and chemical/biological signals. Java generics are used to ensure that only compatible models are interfaced together.

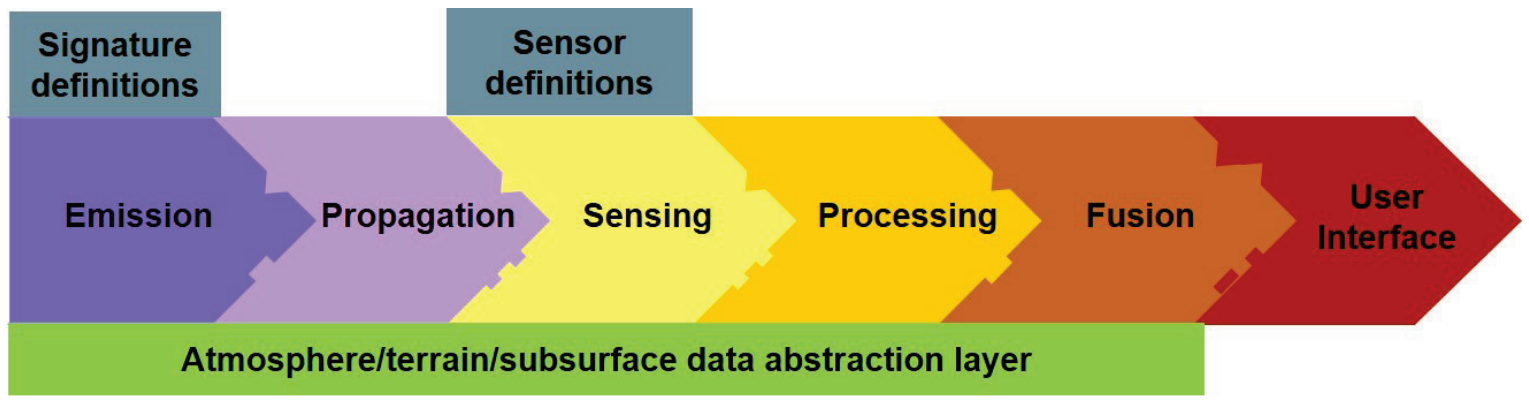

Another key ingredient of EASEE is its environmental data abstraction layer. By environmental, we refer here to the atmospheric, terrain (surface), and subsurface properties. By data abstraction layer, we mean a self-contained data model that unambiguously describes the properties of interest. The information passed between the modeling stages shown in Figure 1 consists of statistical representations for signals and noise (namely, probability density functions [pdfs]) and inferences based on those statistics (e.g., probabilities of detection and false alarm).

Before the current effort, EASEE evolved through two basic versions. The first version, EASEE version 1 (v1), for which the technical report by Wilson et al. (2009) is the primary reference, defined the basic object-oriented software architecture and information exchanged between the components of the modeling framework. The second version, EASEE v2, for which the technical report by Wilson and Yamamoto (2014) is the primary reference, introduced many new features, including an advanced Java programming technique called generics, which resulted in a mature, robust software framework. The generics constructs efficiently ensure compatibility between modeling capabilities incorporated into EASEE. In effect, they declare the scope for a modeling capability. For example, propagation models for optics can only be used for optics (and not, say, seismics), and only acoustic emission models can be linked with acoustic sensor models.

Nonetheless, in both v1 and v2 incarnations, EASEE had a very significant limitation: it predominantly supported modeling in environments with one-dimensional (1D) structure. Specifically, the atmospheric and subsurface profiles were assumed to depend only on the vertical coordinate; that 
is, horizontal stratification was assumed. An exception was the terrain elevation and land surface properties, which were allowed to vary in the horizontal coordinates.

The primary purpose of the Signal Propagation in Uncertain and Complex Environments (SPRUCE) project described in this report was to create a suite of three-dimensional (3D) signal and sensor performance model capabilities for realistically capturing propagation physics in urban, mountainous, forested, and other complex environments. In particular, the environmental model in EASEE was to be extended to include full $3 \mathrm{D}$ variability in the atmospheric and subsurface structure, and to support signal propagation modeling capabilities that leverage this representation. These improvements were designated EASEE v3.

\subsection{Objective}

The objective of this report is to describe the main technical innovations and functionality of EASEE v3.

\subsection{Approach}

Chapters 2 and 3 discuss high-level changes in the structure of EASEE and its interface. Specifically, Chapter 2 describes extensive restructuring of the code into separate Java packages based on sensitivity and release controls for certain capabilities and data. The restructuring also helped to facilitate maintenance and distribution of EASEE-related products. Chapter 3 describes the development of a web service and other support for interfacing with EASEE from external applications.

Chapters 4-6 describe significant modeling enhancements in EASEE v3. In order, these are enhancements to the platform models (i.e., the models for battlefield entities that emit and/or sense signals), enhancements to the environmental representation to support inhomogeneous terrain and $3 \mathrm{D}$ atmospheres, and enhancements to the signal propagation models to support calculations with complex $3 \mathrm{D}$ environments.

Chapter 7 provides conclusions and the envisioned way forward with EASEE. 


\section{Code Refactorization}

EASEE incorporates military signature and sensor data that have distribution limitations outside of the Department of Defense (DoD). Also, some of the modeling capabilities enable signal detection calculations that may be sensitive. Before EASEE v3, the software and data consisted of a single Java project. To address the varying sensitivities of components of EASEE, we partitioned the software into two separate Java projects. The refactorization (programming terminology for rewriting or restructuring existing code to improve its non-functional attributes) helps to protect sensitive technical data while simultaneously facilitating collaboration with agencies and institutions outside of DoD.

The first of the two now-separate Java projects, called KNEE (KNEE is not EASEE in its entirety), is a stripped-down, lightweight version of EASEE, comprised almost entirely of Java code with very few external dependencies. All EASEE capabilities related to the environmental representation (data abstraction layer) and manipulation of geographic coordinates are found in KNEE. Note that KNEE is marked with Distribution Statement C, meaning that distribution of KNEE is limited to U.S. Government Agencies and their contractors.

The second project, called EASEELib, contains the militarily sensitive, proprietary, and export-controlled components, along with our most advanced propagation models and other capabilities used to perform highfidelity calculations of sensor performance. Note that EASEELib is marked with Distribution Statement D, meaning that distribution is limited to DoD and U.S. DoD contractors.

In terms of the software structure, KNEE is a dependency of EASEELib (that is, EASEELib requires KNEE to run) but not vice versa. Figure 2 illustrates this software structure. KNEE functions as an independent module that houses many of the most fundamental interfaces and abstract classes available within EASEE. EASEELib then leverages and, in some cases, extends those interfaces and classes. In this manner, EASEELib builds on some of the fundamental building blocks in KNEE to provide advanced capabilities for modeling signal emission, propagation, and sensor fusion. 
Figure 2. Conceptual diagram of EASEE's software components. EASEELib encapsulates KNEE (a stripped-down version of the EASEE software) and houses many advanced modules that provide enhanced capabilities (purple boxes). EASEELib is also able to facilitate interaction with a host of interfaces (orange boxes).

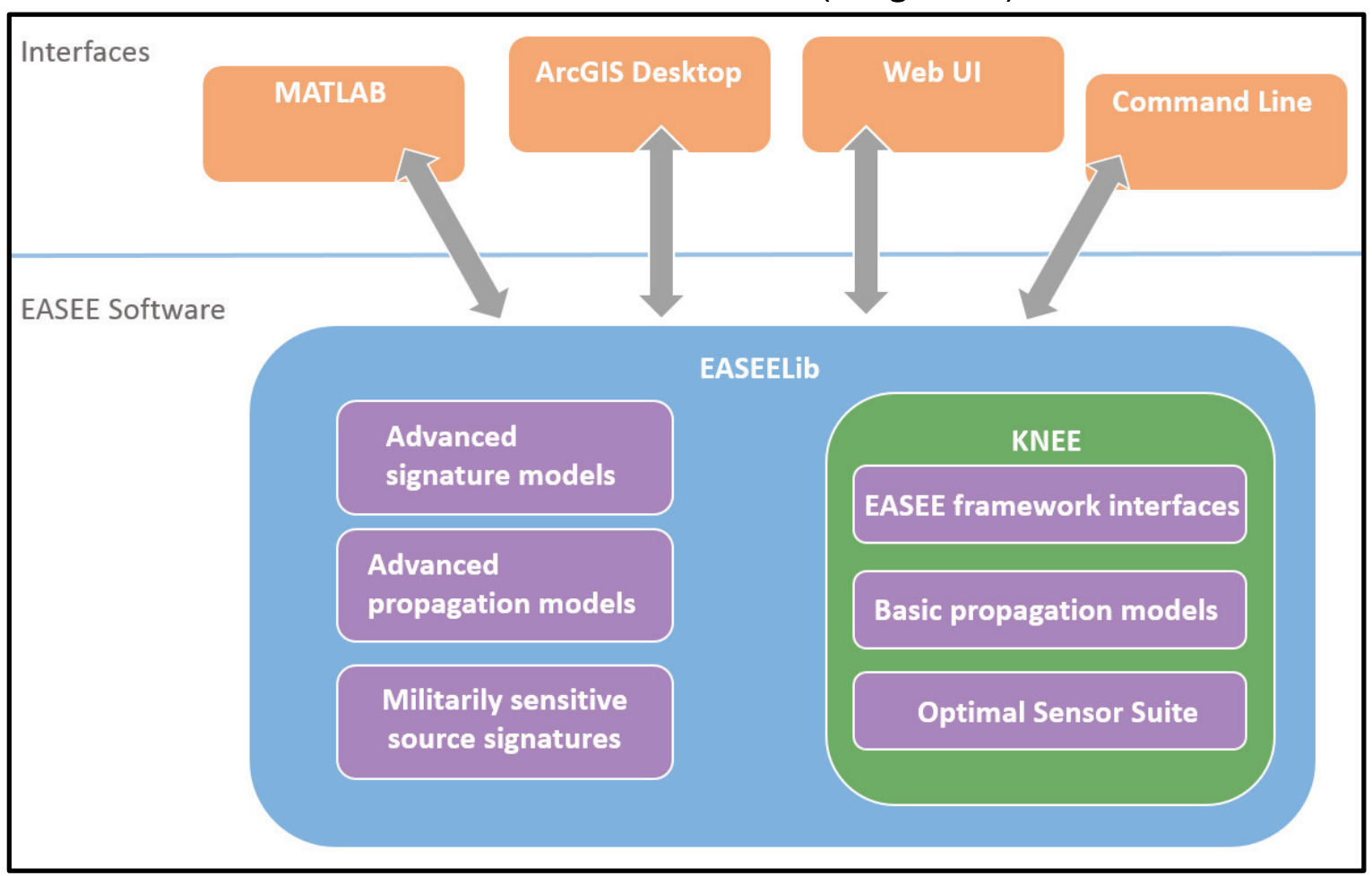

The KNEE project also hosts simple acoustic, seismic, and optical calculations. The acoustic and seismic calculations in KNEE are very basic, with geometric spreading, attenuation, and ground impedance. The optical calculations in KNEE use line-of-sight (LOS) and probabilistic LOS modeling; hence, they do not involve any target characteristics or sensor data. The EASEELib project hosts higher-fidelity acoustic and seismic calculations that include weather and seismic profiles. The optical calculations in EASEELib leverage advanced atmospheric transmission models such as Moderate Resolution Atmospheric Transmission (MODTRAN) and performance models for visible and infrared (IR) sensors. The entire suite of RF capabilities is in EASEELib only.

Additionally, as part of the SPRUCE project, the EASEE code was moved from Defense Information Systems Agency's (DISA's) web-based collaborative software development environment, ProjectForge, to an instance of GitLab hosted at ERDC's Information Technology Laboratory (ITL). The primary reason for the transition was cost savings, since DISA was to begin charging fees for ProjectForge, whereas the ITL site was free to the project. In addition to a change in hosting services, we also transitioned 
EASEE software products (to include KNEE and EASEELib) from a Subversion (SVN) version control system (VCS) to a Git VCS. The conversion from SVN to Git was desirable from the standpoint of leveraging the many advanced features of Git for code management including decentralized development, support for cheap feature branch workflow, and faster release cycles. The transition was completed smoothly, with little to no impact to ongoing projects. To allow for continued collaboration with entities outside of DoD, we host KNEE on ERDC's "public-facing" GitLab instance, which can be accessed by approved users outside of the ERDC, provided common access card (CAC) credentials are available. EASEELib, however, is maintained on a GitLab instance hosted behind the Defense Research and Engineering Network (DREN) firewall.

EASEE currently integrates several proprietary and restricted distribution models such as Electromagnetic Propagation Integrated Resource Environment (EMPIRE), a suite of propagation and sensor models for RF receiving antennae; Multi-Service Electro-optic Signature (MuSES), a software package for modeling the emission characteristics of IR platforms; and MODTRAN, which is used to calculate atmospheric transmission from environmental input. After moving EASEE to GitLab, the code repository was restructured to separate the proprietary models from the master EASEELib project to facilitate distribution of EASEE without these components. The separated components are retained in the overall code base due to their research value and possible employment in future, servicebased applications. 


\section{EASEE Interface and Web Service}

This chapter describes approaches to interfacing with EASEE from external applications. The reader is referred to Waldrop et al. (2017) for a more extensive discussion, including our efforts to open the EASEE interface to a broader community of software developers.

\subsection{XML schema}

Extensible Markup Language (XML) is a widely used language for exchanging information between computer applications. XML is extensible in the sense that only the basic format and commands are standardized; the names of the fields and values appearing in a valid XML document are defined by a customized XML Schema Definition (XSD). The basic XML information flow is that one application will populate an XML document and push it to a receiving application, which validates it against the XSD and extracts the information of interest.

An XML-based interface was originally created for EASEE v2. The key component of the interface is the EASEE XSD, which defines information on the type of calculation to be performed, the environmental representation, the coordinate grid for the calculation, the platforms (emitters and sensors), and the signal propagation models to be used, among other options. We overview some of these elements as background for the discussion that follows in this section.

The XSD defines several calculation types: probability of detection, probability of false alarm, optimal sensor placement, optimal sensor activation, signal strength, and noise strength. It also defines the coordinates for the area of interest, grid resolution of the area of interest, and specific features associated with platforms, such as category, geographic coordinates, and altitude, to name a few.

To account for terrain effects on signal propagation, the EASEE XSD can ingest a digital terrain model (DTM), a digital surface model (DSM), landcover data, and soil data. The landcover and soil can be specified as single types across the entire domain or with files describing their spatial variation. Atmospheric profiles are specified with a wind class and a stability (thermal stratification) class. The XSD also supports specification of the 
background noise models for the acoustic, seismic, very high frequency/ultra-high frequency (VHF/UHF), and super-high frequency/extremely high frequency (SHF/EHF) ambient noise.

EASEE incorporates many complex, physics-based models for signal modalities, including acoustic, infrasonic, seismic, RF, IR, and visible. Since end users often have little understanding of the characteristics that distinguish one propagation model from the next, the EASEE XSD provides a layer of abstraction so that models are selected indirectly based on fidelity and, therefore, computation time. For each modality, users can select a low, medium or high-fidelity calculation, and the EASEE Java library will select a propagation model that most closely resembles the fidelity. To further refine calculations well suited to a specific use case, the EASEE XSD allows users the ability to enable or disable any number of the signal modalities.

\subsection{Interfacing with the ArcGIS Desktop}

The first graphical user interface (GUI) developed for EASEE, the EASEE MatlabGUI, was based on the Matlab Mapping Toolbox. The EASEE MatlabGUI can be compiled into a standalone Windows executable and distributed to end users who do not have a Matlab license. While the MatlabGUI is still in use today and has robust functionality, it was designed primarily for research or advanced users and, thus, presents limitations to non-expert pilots and planners who wish to use EASEE for mission planning.

ArcGIS is the GIS software most commonly used by Army terrain analysts at the division and brigade levels and by various intelligence community users. In fact, we have found that some EASEE users employ the EASEE MatlabGUI to run calculations and then import the raster output into ArcGIS Desktop applications for further analysis. To provide a seamless way to access EASEE from ArcGIS, we developed an EASEE toolbar for ArcGIS Desktop.

At the outset of the development, design objectives for the ArcGIS user interface (UI) were evaluated. Of the many requirements, an especially high importance was placed on seamlessly integration with the ArcMap environment from a visual perspective and the association of trigger events (e.g., mouse selections) from the toolbar to real-time visual effects in the 
ArcMap environment. Both objectives were realized by crafting an Esri Python add-in, which is an ArcGIS Desktop plug-in that unites similar tools, buttons, and combo-boxes via a toolbar.

The design of UI widgets was tailored according to the following: elements available in the XSD and data for which we knew our end users would have access. The final UI product shown in Figure 3 maintains combo-boxes for specifying the calculation type, scenario, situation, and grid division; tools for interactively defining an area of interest on the map or placing fixed platforms; and buttons that trigger Python script tools, allowing users to input text-based configuration settings for signal modalities, signal propagation fidelities, terrain, landcover, soil, atmospheric conditions, and background noise. Figure 4 shows an example of a pop-up that results after clicking a button on the toolbar.

Figure 3. EASEE ArcGIS toolbar, which integrates with ArcMap and allows users to select specific configuration settings for an EASEE calculation.

\begin{tabular}{|l|}
\hline EASEE \\
Calculation Type Probability of detection \\
Scenario Vulnerability \\
Situation Hostile monitoring friendly \\
Grid Division $400 \times 400$ \\
\hline
\end{tabular}

Figure 4. Input form pop-up triggered by the Landcover and Soil button on the EASEE toolbar. This is just one example of an input form. Others are available for selecting signal modalities and fidelities, terrain, atmospheric conditions and background noise, and fixed and variable platform attributes.

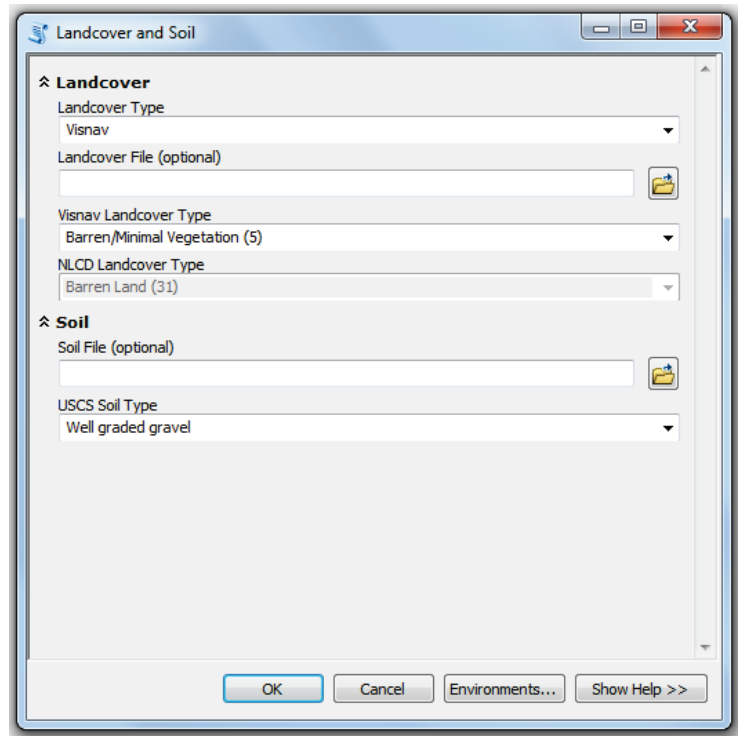


ArcGIS Desktop provides geoprocessing system tools that can manipulate or transform geographic data. In most cases, users supply input to the tool, often in the form of a pop-up as described in the previous section. From there, a geoprocessing script is executed, and the associated output is made available to the user. In addition to pre-configured system tools, Esri grants developers the opportunity to implement their own geoprocessing script tools. We exploited this capability to gather user-defined configuration settings for an EASEE calculation. Instead of executing a geoprocessing script, our scripts take user-defined selections and store them to Python class definitions generated from elements of the EASEE XSD via a Python module called generateDS. Python class definitions may seem like unnecessary overhead. However, they are extremely useful when it comes time to run an EASEE calculation since each class maintains an export method, the output of which adheres to the EASEE XSD, thus certifying the validity of any XML document that is passed from ArcGIS to EASEE.

The ArcGIS Desktop requests an EASEE calculation by first exporting data from Python classes to a valid XML string. Then, it instantiates a Python subprocess that runs EASEE via the system's Java Runtime Environment, passing the XML string as a parameter. EASEE receives, reads, and validates the XML string and performs the type of calculation specified within the XML. Next, EASEE writes the results to a KML (Keyhole Markup Language) file accessible by ArcGIS Desktop. ArcGIS Desktop interprets the KML, performs some small manipulations on the data, and loads a raster file into the user's workspace.

Figure 5 shows a demonstration of the general architecture and flow of communication between EASEE and the ArcGIS Desktop application. The EASEE_Python_addin Python module maintains classes associated with each UI widget on the EASEE toolbar. Methods within each class provide implementation details on how events such as a button click are handled. In some cases, a button click warrants the execution of a Python script tool that provides the user with a pop-up UI for selecting EASEE configuration settings as shown in Figure 4. If updated, these values are pushed to fields in corresponding Python classes. If the user selects the "Calculate" button from the toolbar menu, the communication protocol outlined above is executed. 
Figure 5. Demonstration of the general architecture and flow of communication between EASEE and the ArcGIS Desktop application.

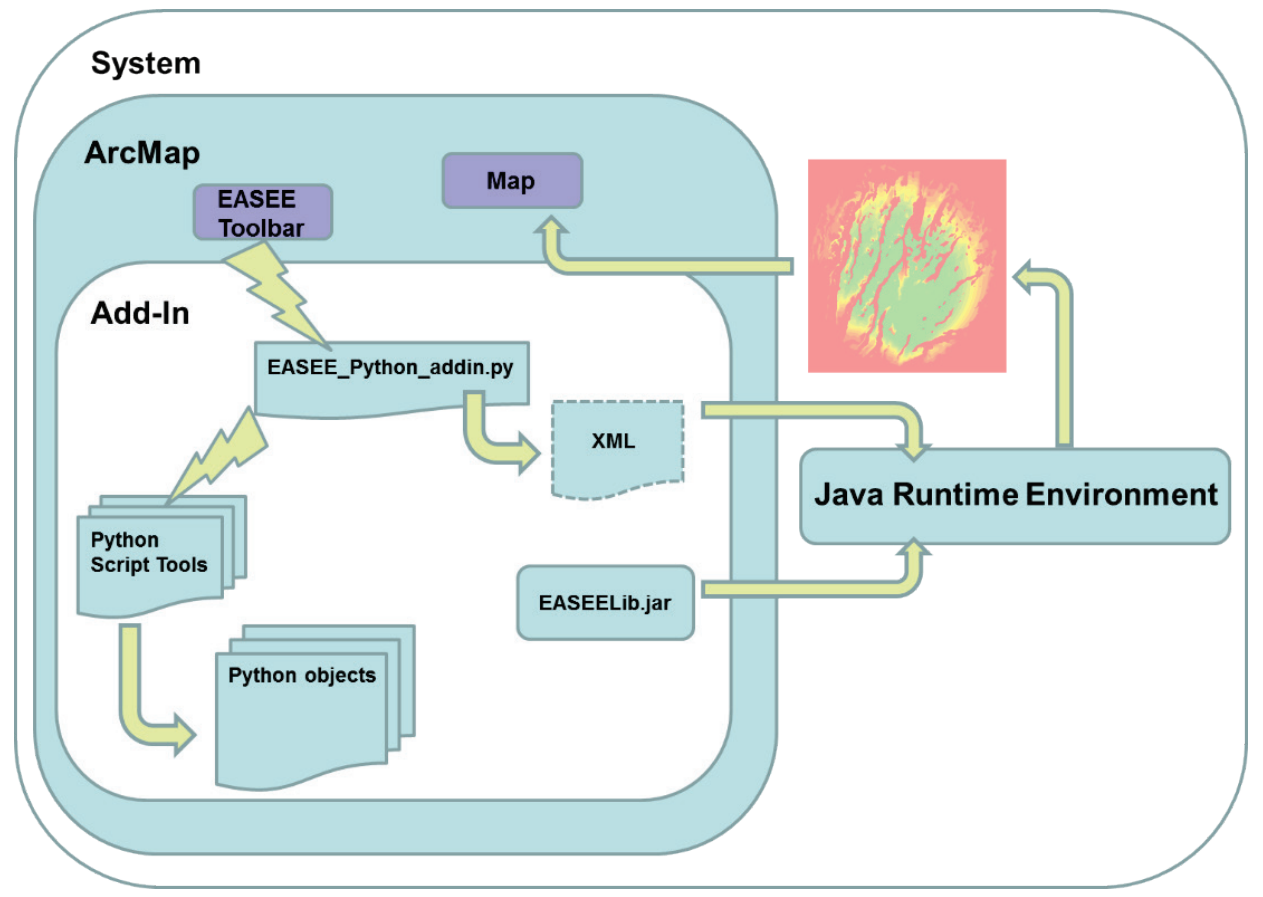

Figure 6 demonstrates output associated with an EASEE calculation run in a $5 \mathrm{~km} \times 5 \mathrm{~km}$ region of Fort Huachuca, AZ, in the context of the ArcGIS Desktop application. In this example, a probability of detection calculation was run in which we wanted to address the following question: "What is the probability that a fixed, attack helicopter (emitter) can be sensed by a hostile human listener on the ground?" This question warrants the selection of "Vulnerability' from the Scenario combo-box and a corresponding 'Hostile monitoring friendly'selection from the situation combo-box. In this sample calculation, the acoustic modality was enabled while all others were disabled, and a high acoustic signal propagation fidelity was selected. Additionally, the simulation considers the terrain; however, properties associated with atmospheric conditions, and background noise were left to default values.

As Figure 6 demonstrates, the output generated by the EASEE calculation engine is a layer that nicely meshes with other feature layers available in the user's table of contents in ArcMap. In addition, fixed platforms correspond to features in a Platform feature class. Platform features and their associated attributes can be modified just like any other point feature created in ArcMap. While not captured here, it is important to note that one of the primary benefits of running EASEE calculations from ArcGIS Desktop is the potential to integrate EASEE output with other Army ArcGIS Python add-in products, all from the convenience of a single GIS environment. 
Figure 6. Output corresponding to a probability of detection calculation run in EASEE. The green areas are indicative of a high probability of detection of the helicopter (annotated as a blue diamond) by a hostile human listener on the ground, while the red is indicative of a low probability of detection.

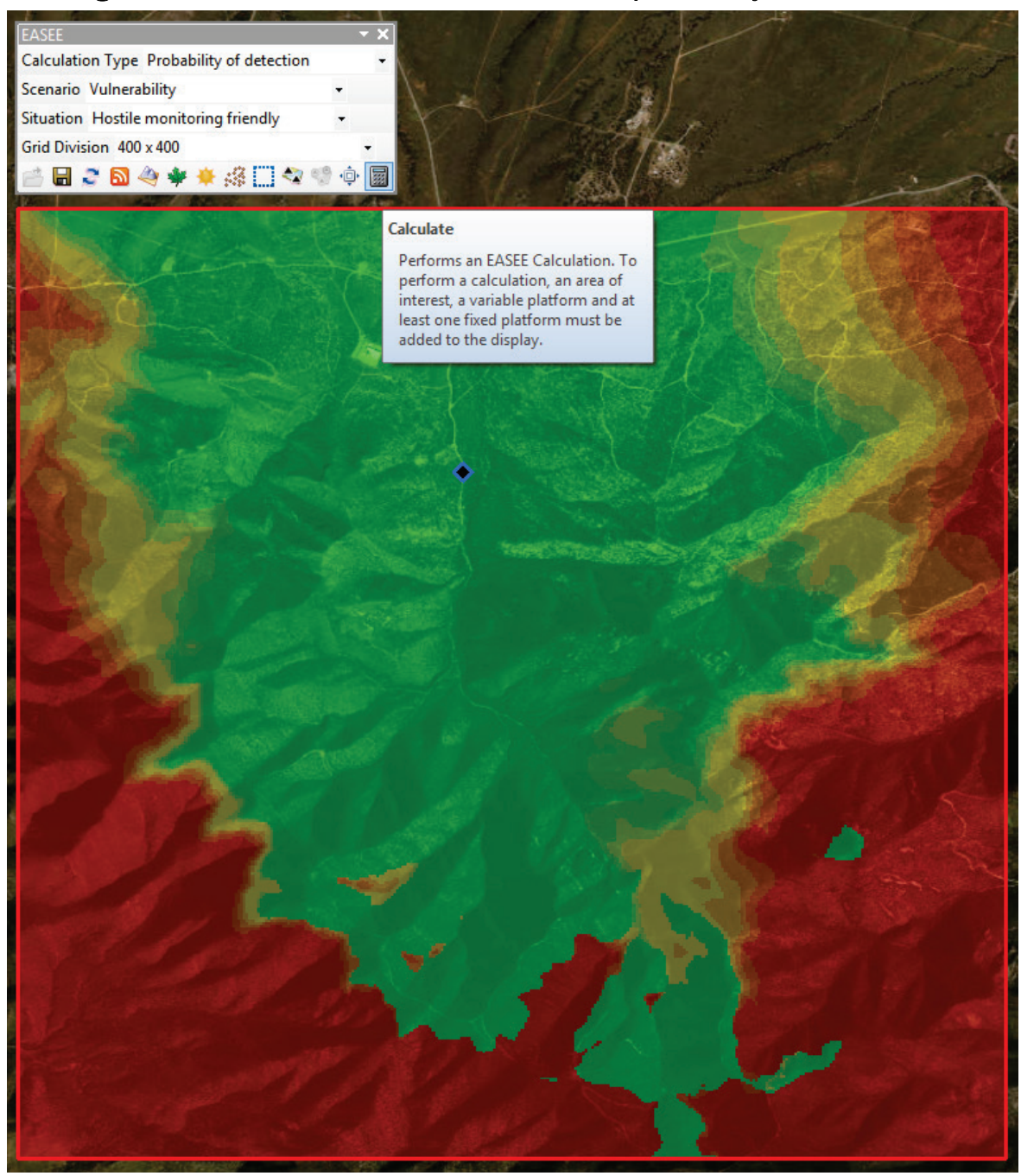

\subsection{EASEE web services}

EASEE Web Services (EWS) enhance EASEE by enabling the creation and delivery of sensor performance products to systems in the Army Geospatial Enterprise, aligning the software for compliance with the Army's Common Operating Environment (COE) and with the ultimate objective of operating and interacting with a broad range of Army Computing Environments (CEs).

By following the principles of Modular Open System Architecture (MOSA), we have enabled the high-fidelity physical models to better operate with systems in the COE and enhanced the capability with web-service and geo- 
spatial enterprise services technology that will align with current and future Army Command Post CEs and Sensor CEs. The advantage of this approach is threefold:

- Enhanced adaptability. The technology, realized in a web-service and thin-client architecture, can evolve and be rapidly modified to meet the changing needs of the data consumer and evolving use-cases with limited required software engineering or redeployment of the core services. This includes both the front-end adaptability where the UI can support different use-cases, but also leveraging web-service back-end interfaces that can respond to a diverse set of client requests for sensor performance products.

- Interoperability. The software can be readily integrated with other services in an enterprise that both ingests data from other systems and produces data to support other services or end consumers. The geospatial data services use Open Geospatial Consortium (OGC) compliant open standard interfaces that enable Army geospatial data providers to readily provision the system with data, but also enable OGC ${ }^{\circledR}$ compliant consumers to use the output products.

- Portability through hardware independence. The capability can be deployed in a broad spectrum of target systems without any requirement for recompilation. The thin-client requires only a web-browser to execute, and the service code is written almost solely in Java, making the entire system highly portable. Typical deployments leverage system virtualization, where we use immutable infrastructure to codify system configuration and automate system builds.

\subsection{Simple Object Access Protocol (SOAP) and Representational State Transfer (RESTful) endpoints}

EWS provides web-service end points using the popular SOAP and RESTful (Representational State Transfer) protocols. Any client application using the EWS SOAP service can submit a SOAP request that provides details of the underlying EASEE calculation to be performed (available sensors, target, areas of interest, weather conditions, etc.) and, in return, receives a response that contains a series of OGC-compliant Uniform Resource Locators (URLs) hosted by a Geoserver instance, where the EASEE output data and products can be retrieved. This technology employs a mature, commercial industry standard web-service process in which requests are made using the Hyper Text Transfer Protocol (HTTP) that conform to the Web Service Definition Language (WSDL) that is published with the 
EWS service. The WSDL strictly defines the input parameters required to make a successful request as well as the parameters to expect in the response. EWS also uses a RESTful framework that can receive a XML payload that adheres to the WSDL. Alternatively, simplified REST endpoints can be implemented that capture the parameters needed for tailored client use-cases.

The production and publication of sensor performance decision products use OGC-compliant services that align EWS with the Army's Command Post Computing Environment (CP CE) Version 3 Software Requirements and with the requirements for Standard Shareable Geospatial Foundation (SSGF) data, which place an emphasis on interoperable GIS data through OGC-compliant interfaces. Figure 7 shows the data interfaces for a typical EWS deployment configuration. The left side of the figure shows any number of EWS WebUI clients, or third-party GIS systems, using the SOAP or REST interfaces to initiate calculations. The right side shows services that provide map imagery, weather data, and terrain information. Figure 8 shows the EWS product data interfaces.

After EASEE produces a calculation, EWS publishes the data to a Geoserver instance and responds to the requesting client with URLs for each of the data services. The data is potentially made available to any system that can access those URLs. This set of services enables any system that aligns with MOSA, COE, and SSGF requirements to generate the high-fidelity sensor performance products produced by EASEE and to automatically disseminate those products to other systems over the network.

Figure 7. Input data interfaces for the EASEE web service.

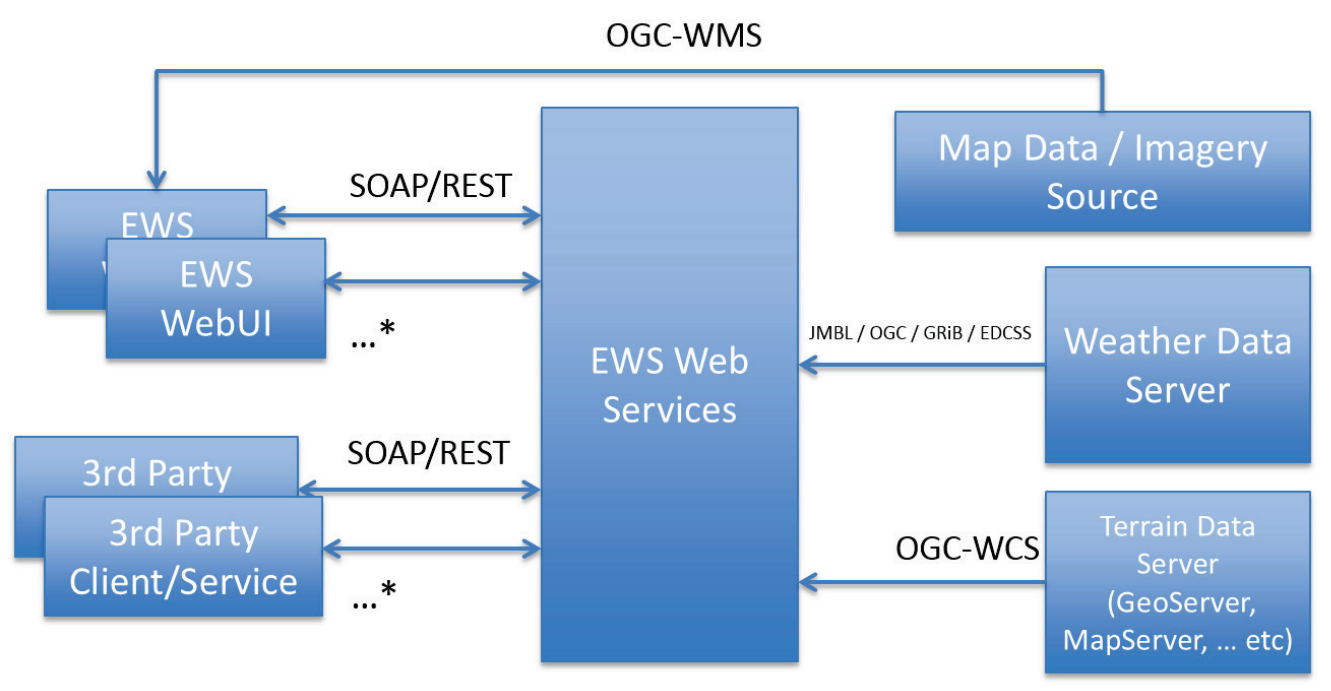


Figure 8. Output product interfaces for the EASEE web service.

EASEE Data / Products via OGC-WMS,WCS,WFS

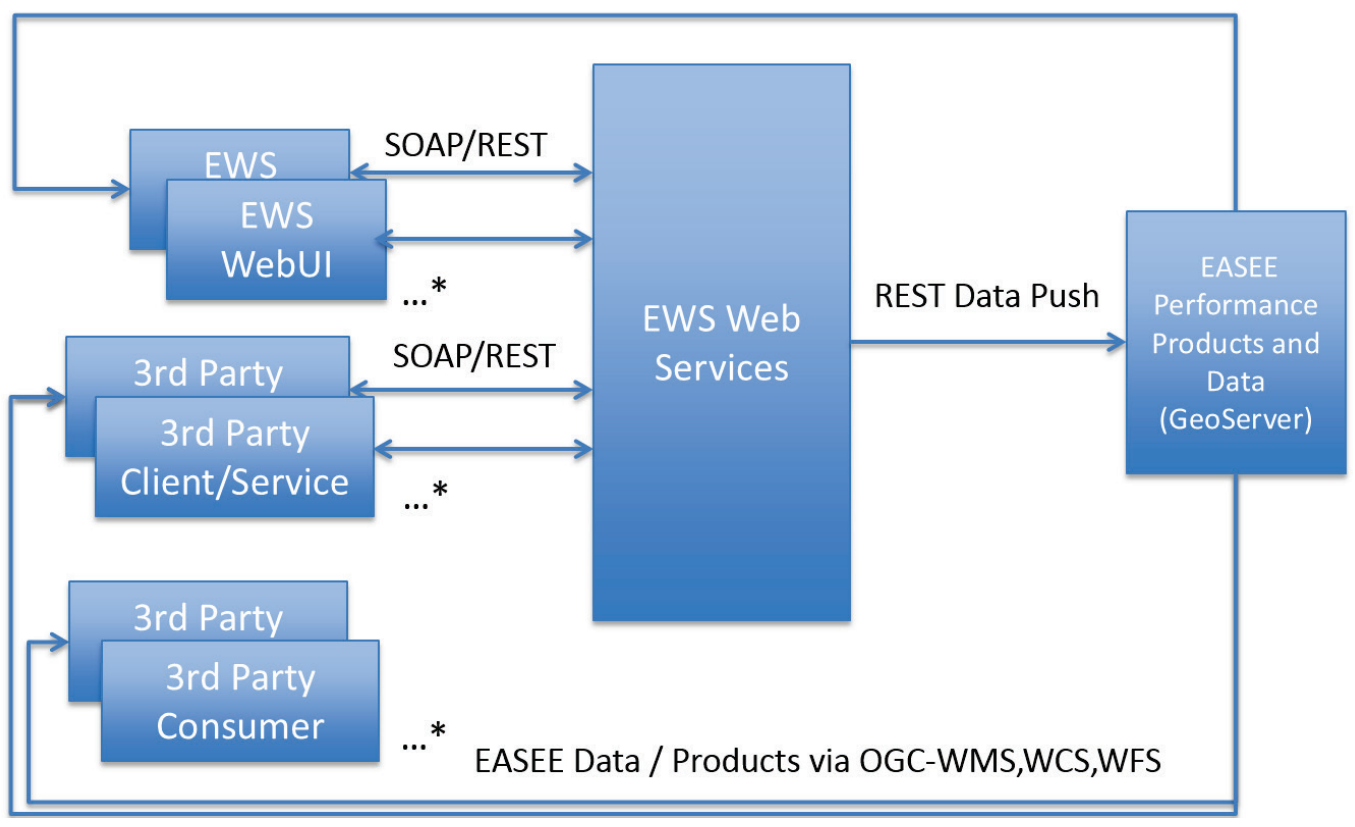




\section{Platform Enhancements}

\subsection{General}

Platform objects in EASEE represent battlespace entities such as humans, ground vehicles, air vehicles, and sensors. They encapsulate modules representing the emission, sensing, and processing capabilities of these platforms (Figure 1). (Propagators, however, are set up as "services" shared by all the platforms.) Platforms also have a state describing the position, velocity, and orientation of the platform. The state may be specified at multiple points in time.

In keeping with object-oriented programming principles, platforms in EASEE are designed with flexibility and reuse in mind. EASEE maintains an abstract superclass, called Platform, which serves as the basis of all other platform classes, and defines states and behaviors common to platform categories. The abstraction avoids duplication of code, encourages reuse and simplifies code maintenance since updates applied to the superclass are automatically applied to the corresponding subclasses. When developers wish to add a new platform category to EASEE, the implementation is as simple as extending the Platform superclass, defining new methods or fields specific to that platform category, and if necessary, changing or extending inherited behaviors.

The non-abstract platform subclasses represent battlefield entities with similar signal emission and sensing characteristics (e.g., helicopters, fixedwing aircraft, Unattended Ground Sensors [UGS]). Within each of these subclasses, several platform types are defined that represent the specific platforms available for calculations. For example, within the Helicopter subclass, types are defined for generic small, attack, and transport helicopters, and for specific military helicopters such as the Apache (AH-64), Blackhawk (UH-60), Chinook (CH-47). Figure 9 shows the relationship between the abstract Platform class and the Helicopter subclass, along with other general relationships between the abstract Platform class and other non-abstract subclasses. 
Figure 9. Relationships between classes associated with platforms in EASEE. This diagram provides a simplified view of the essential fields and methods available within each class and the PlatformTyping interface. The focus is primarily on the relationships between component parts as opposed to the contents of each component.

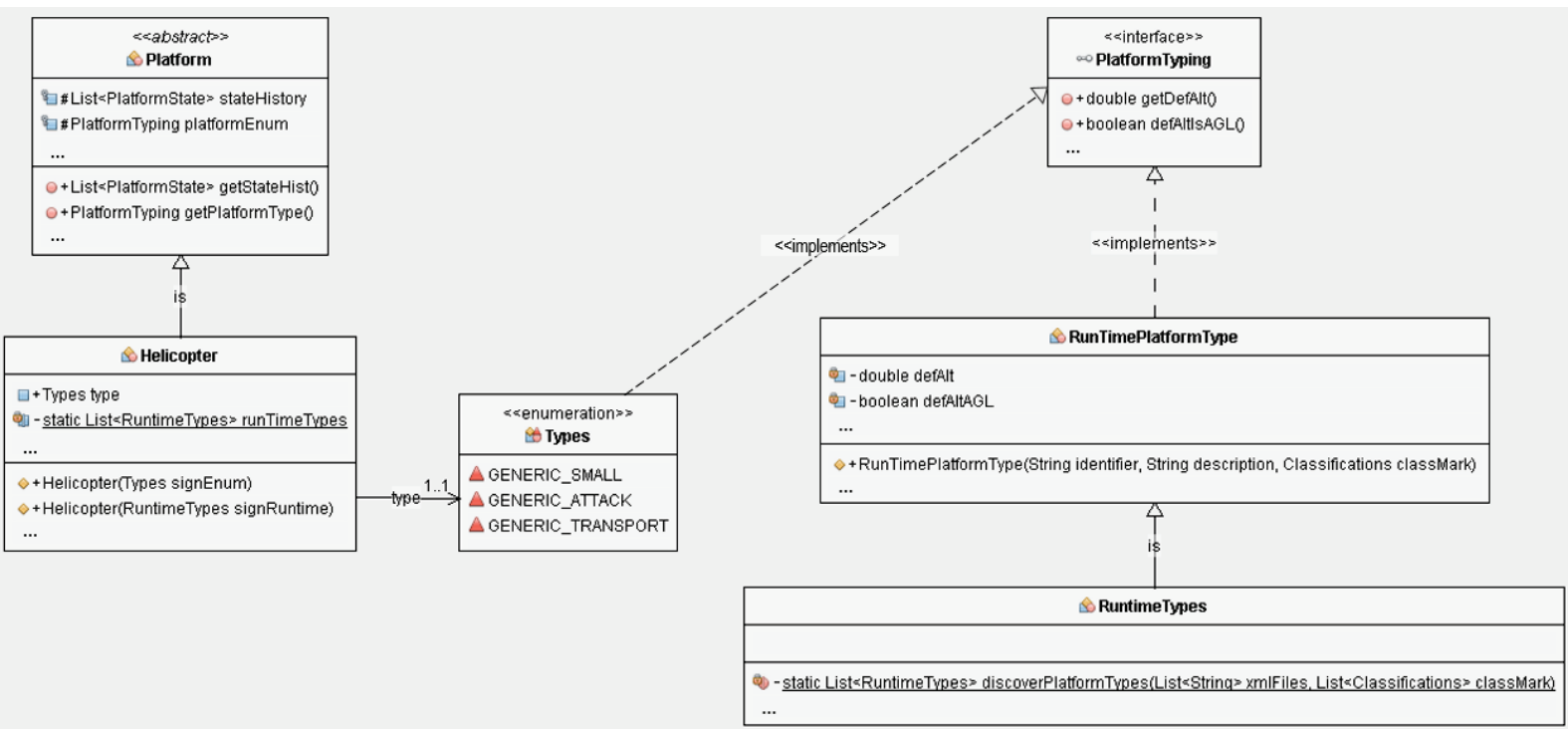

\subsection{Dynamic loading of platform signatures}

Another innovation was the addition of a capability for dynamic "discovery" and loading of new target signatures via XML files for platform types via XML files. This enables ingestion of new signatures into EASEE without recompilation of the code, which is a particularly valuable capability when users wish to customize the entities modeled by the software. It also facilitates packaging of versions of EASEE without these signatures, thereby safeguarding Army technical data. This may be necessary when dealing with signatures having distribution limitations (e.g., export-controlled data).

Before EASEE v3, every platform type in EASEE was hard-coded within each platform class using Java enumerations. The enumerations were defined as Java inner classes within each non-abstract Platform class. Thus, only these platform types were available in compiled versions of the code. Since it is impractical for end users to recompile the code, we sought an additional method for defining platform types that would enable users to add their own platforms and associated signatures. To this end, we adopted an XML-based approach for data exchange and validation to dynamically import platforms and properties with distribution restrictions at runtime. The gist of the dynamic loading is that EASEE looks for files called "platforms.xml," which are XML-format files that list the signatures that the user wants to load into EASEE. A different folder is searched for each 
platform class. The platforms.xml file, when present, specifies the name of the platform type to be added, the name of the file where the signature is located, and the format of the signature data. Among the signature data formats are octave bands, one-third octave bands, and arbitrary frequency bands with specified center frequency and bandwidth.

The following outline describes the specific code upgrades involved:

- Platform XML documents were created for each platform category maintaining enumeration types associated with controlled unclassified data source signature files. Each platform XML document identifies several XML elements associated with platform properties, e.g., descriptive name, the path for the source file, and default altitude.

- An XSD was created to formally outline the "allowable" elements within platform XML documents. The XSD is used as a mode of validation to ensure that all XML documents adhere to certain specifications.

- The schema was bound to a set of Java classes representative of the schema by leveraging the Java Architecture for XML Binding (JAXB). The JAXB Application Programming Interface (API) allows developers to access content in XML documents from Java.

- Hard-coded enumerations associated with controlled unclassified data source signature files were removed from the source code.

- For each platform category, a Java static initializer was implemented to discover platform types in an XML file at runtime.

At runtime, EASEE attempts to find new platform types in XML files for each available platform category with a static initializer, which calls the method discoverPlatformTypes in the RuntimeTypes class. (See Figure 9 for a reference of the RuntimeTypes class and how it relates to other platform classes in EASEE.) For each XML document, a JAXB unmarshaller parses the XML file and creates a new RuntimeTypes object that gets appended to a list of RuntimeTypes objects. The list, in turn, gets assigned to the static RuntimeTypes list available in each platform category class (e.g., Helicopter). Additional methods in each platform category class combine both hard-coded types with unclassified signatures and runtime types with controlled unclassified signatures to complete the set of specific platform types available.

Simply removing platform XML files and corresponding controlled unclassified data source signatures from the JAR (Java ARchive) file before deployment ensures that deployed products adhere to export control guidelines and facilitates distribution of EASEE to organizations outside DoD. 
Another feature added to EASEE as part of the capability to dynamically load signatures was the ability to automate back-propagation signatures. By back-propagation, we mean the compensation of the signature for the conditions at the time of the measurement. Specifically, the back-propagation algorithm, as currently implemented, compensates for the separation distance between the source and receiver, based on the assumption of spherical spreading. The goal of the compensation is to determine the level that would be observed, in principle, in free space at $1 \mathrm{~m}$ from the source. (One could also attempt to compensate for the ground and atmospheric conditions and other factors, but this is much more complicated and prone to uncertainties.) The signature data can also be automatically denoised, i.e., the background noise level can be removed. These automated capabilities help enable non-expert users to incorporate their signature data collections.

\subsection{Source and receiver directivity}

Many new features were incorporated into EASEE $\mathrm{v} 3$ to support directional source emissions and sensor responses (e.g., RF antennae and acoustic microphone arrays). The directivity functions can have an arbitrary dependence on the two spherical coordinate angles, namely the azimuth and elevation relative to the source or receiver.

EASEE's radpat (short for "radiation pattern") Java package provides the functionality for directivity patterns. Figure 10 shows the structure of this package. Two primary classes of radiation pattern specifications are available in the radpat package, both of which are supported by most of the acoustic and RF propagation calculations in EASEE. These are RadiationPatternHarmonics, which describes the radiation pattern in terms of spherical harmonics, and the abstract class RadiationPatternAngular, which specifies the functional dependence of the radiation pattern on the two spherical angles directly. RadiationPatternAngular is implemented in two primary ways. First, RadiationPatternBeam specifies a single beam or field-of-view, which is defined by the azimuth and elevation beam center angle and the angular extent with respect to these two angles. Second, RadiationPatterRaster and RadiationPatternHemi specify the radiation pattern as a discrete grid (raster), which is two dimensional (2D) in the azimuth and elevation. They differ in the orientation of the coordinate systems, as will be discussed shortly. 
Figure 10. Inheritance tree for the EASEE representations (Java classes) for directivity functions. This functionality may be used for both source radiation and sensor beam patterns. The classes shown with a white font are abstract; the black font indicates a nonabstract class.

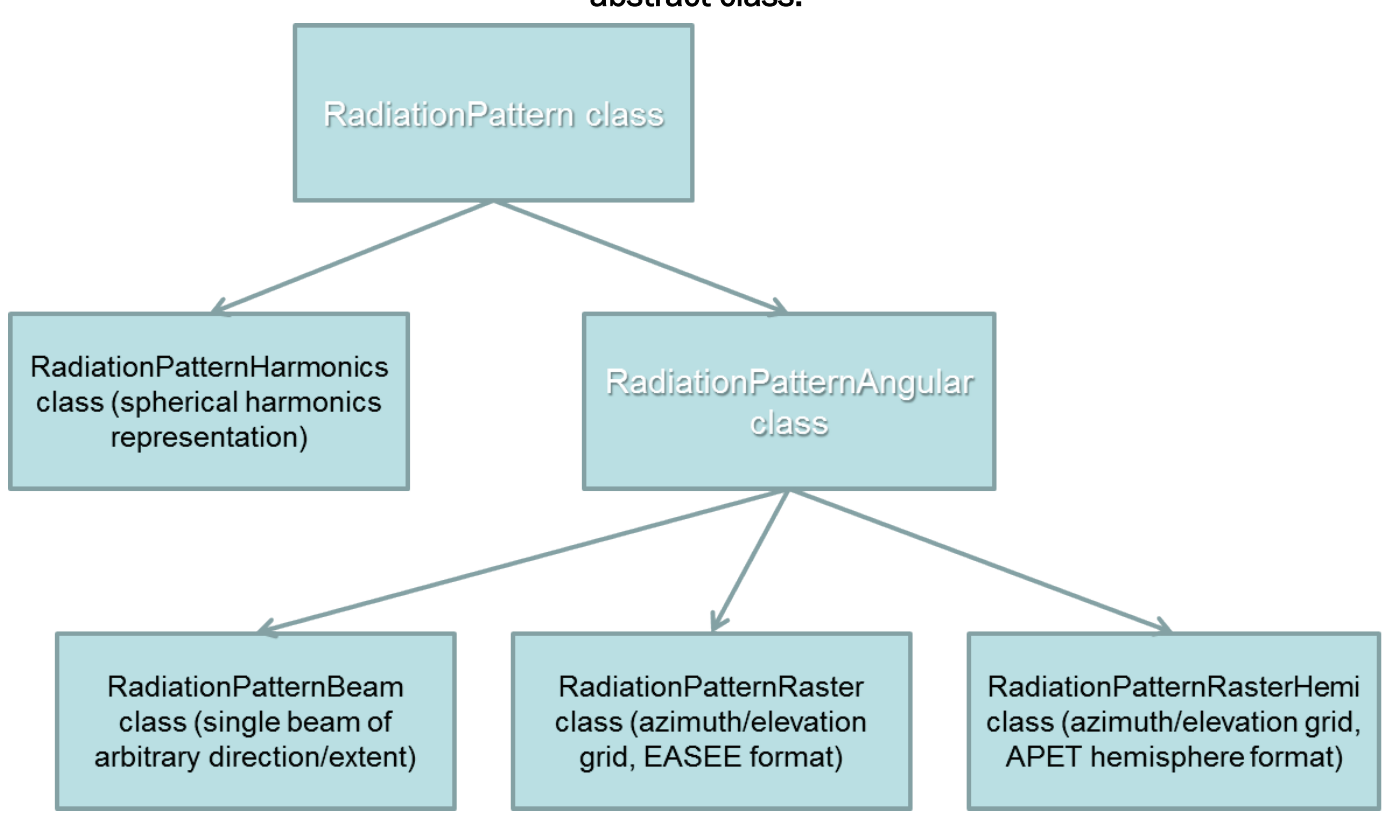

In principle, the spherical harmonic representation can be exactly related to an equivalent point source distribution (Vecherin et al. 2011), which is desirable from the perspective of numerical wave propagation modeling. The angular representations, on the other hand, generally depend on high frequency and straight line (ray) propagation approximations. Such assumptions are usually appropriate for optics but less suitable for the relatively long wavelengths characteristic of acoustic and RF waves.

Figure 11 shows the spherical coordinate system used by EASEE. This system defines the azimuth as the angle in a horizontal plane, along with an elevation angle that is positive for points above horizontal and negative below. This system generalizes the original 2D directivity model in previous versions of EASEE, for which only the horizontal azimuthal dependence was addressed. This system is a natural choice when modeling directivity for primarily horizontal problems. Alternatively, one could specify spherical angles representing the fore-aft angle and the azimuth in a planar cross section perpendicular to the axis of the platform (Figure 11). 
Figure 11. Spherical angle systems used to describe directivity functions in Acoustic Propagation and Emulation Toolset (APET) (left) and EASEE (right).

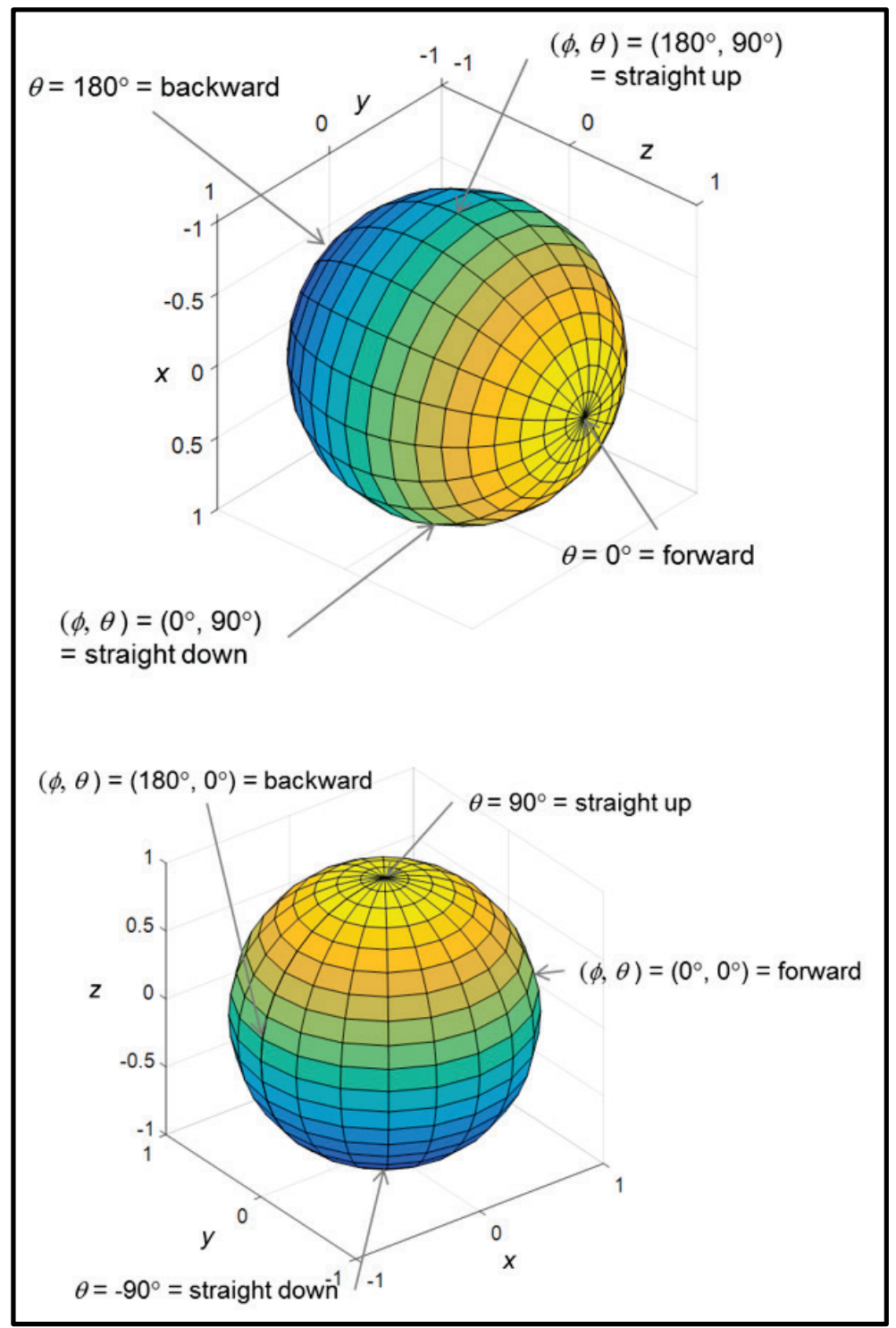

This is a natural choice when modeling directivity for primarily vertical problems as may be encountered with aircraft, since it places the most regular region of the angular grid underneath the aircraft. It is used, for example, by National Aeronautics and Space Administration's (NASA's) APET and Rotorcraft Noise Model software (Page et al. 2008).

To support both coordinate systems, the separate classes RadiationPatterRaster and RadiationPatternHemi were developed as mentioned above. These implement the native EASEE and the APET systems, respectively. RadiationPatternHemi loads rasters from APET source files and stores them directly. When the radiation pattern needs to be evaluated at an azimuth and 
elevation specified in the EASEE spherical coordinate system, these angles are mapped to the corresponding APET angles. A 2D linear interpolation is then used to find the radiation pattern at the desired APET angles.

\subsection{Coordinate rotations}

Rotations of platforms, including their directivity functions, were also incorporated into EASEE v3. This feature is particularly important for calculations with aircraft and other platforms that can rotate arbitrarily. The rotations include all degrees of freedom for a rigid body, which are usually called yaw, pitch, and roll. Considering an aircraft as an example, yaw refers to the bearing (azimuth) in a horizontal plane. Pitch refers to the elevation angle relative to the horizontal plane, i.e., nose up or nose down. Roll refers to banking of the aircraft, i.e., the rotation relative to an axis along the fuselage. For ground vehicles constrained to a surface, only rotations in bearing are possible, i.e., the pitch and roll are fixed, and only the yaw angle can vary.

Treatment of rigid-body rotations is complicated by the fact that twelve different conventions are possible for defining the axes and the order in which the rotations are applied. Wikipedia (2019a) provides a thorough introductory treatment of the subject. The approach implemented in EASEE is based on Tait-Bryan angles, which is also called the intrinsic rotation convention. The orientations of the axes and rotation angles follow the aeronautical convention (Wikipedia 2019b). The vertical or $z$-axis runs through the center of the aircraft and is positive downwards. The longitudinal or $y$-axis runs along the fuselage and is positive to the nose of the aircraft. The transverse or $x$-axis is positive to the right (starboard) side of the aircraft. The order of the rotations is first yaw (rotation about the $z$ axis), then pitch (rotation about the $y$-axis in the coordinate system resulting after the rotation in yaw), and then roll (about the $x$-axis in the coordinate system resulting after the rotation in yaw and pitch). This convention corresponds to column 2, line 5 in the table in Wikipedia (2019a).

Note that when the aeronautical coordinate system, which is right-handed, is re-oriented in the convention used for Earth-based Cartesian coordinate systems ( $\mathrm{x}$-axis to the east, $\mathrm{y}$-axis to the west, and z-axis upward), the pitch angle becomes positive for downward rotations. Hence, the elevation angle is the negative of the aeronautical pitch angle. 


\subsection{Human auditory modeling}

As part of a joint project with NASA, the Glasberg-Moore human auditory critical band model (Glasberg and Moore 1990) was integrated into EASEE. This is the model used in NASA's AUDIB software for aircraft audibility.

The human auditory system processes signals in many ways that differ fundamentally from modern electronic systems. There is a long history of modeling human detection of acoustic signals by calculating a d-prime $\left(d^{\prime}\right)$ metric for each band. The approach originated primarily in the 1950 s and 1960 s when most of these calculations had to be done by graphical methods. It is based on questionable assumptions (e.g., Gaussian signals) and approximations that are not necessary with modern computing capabilities.

One particularly important aspect of the conventional calculation method is the presence of the " $\eta$ factor," which represents the acuity of the detector relative to plain power-based processing approaches used in conventional electronic systems; that is, it attempts to account for the many (not entirely well understood) things that the human auditory system does that are not done with conventional signal processing. A value of $\eta$ less than one represents a gain relative to theory. Going back at least to the early 1970 s, a value of $\eta=0.4$ (a $3.98 \mathrm{~dB}$ processing gain) has traditionally been ascribed to humans (e.g., Fidell et al. 1972). Assuming spherical spreading, this would mean that a human can detect an aircraft at a distance 1.6 times farther than the otherwise equivalent prediction for an electronic system.

To adapt the conventional human detection model to EASEE, we can take as the starting point the following equation (Fidell et al. 1972) for the signal power $(S)$ at which the aircraft will be detected:

$$
S=N d^{\prime} / \eta W
$$

where $N$ is the noise power and $W$ is the bandwidth.

We typically set $d^{\prime}=0.362$, which for Gaussian signals and noise corresponds to a detection rate of $50 \%$ and false-alarm rate of $1 \%$. Proceeding in this way, we can set the threshold at which the detector reports a "hit" to:

$$
S+N=N\left(1+d^{\prime} / \eta W\right)
$$


This approach can be readily implemented using the existing relative threshold detection algorithm in EASEE (the threshold being specified relative to the mean background noise level). However, it is important to keep in mind that this approach does not, in the end, produce the specified detection and false-alarm rates. The target values for those rates pertain only to a Gaussian signal model, which is unrealistic in this application. Usually, the signal distribution at the detector in EASEE is modeled as exponential (that is, a deep fading characteristic). In the end, it is does not appear possible to satisfactorily reconcile the conventional detection approach based on the d-prime metric with a more modern signal detection theory that supports non-Gaussian signals.

Another particularly important aspect of the auditory system is its ability to resolve signals in time and frequency. In the literature on psychoacoustics, critical bands are used to model the frequency resolution. The width of critical bands is expressed as equivalent rectangular bandwidth (ERB). EASEE calculations previously assumed the critical bands were one-third octaves, which corresponds to

$$
\mathrm{ERB}=\left(2^{1 / 6}-2^{-1 / 6}\right) f
$$

where $f$ is the band center frequency.

Other more sophisticated models have been devised in the psychoacoustics literature based on auditory experiments. While the ERB is reasonably approximated by one-third octaves at higher frequencies in the audible range, it is know that it is much wider at lower frequencies. The Glasberg and Moore (1990) model is widely used to describe this effect; their equation is

$$
\mathrm{ERB}=24.7(4.37 f / 1000+1) .
$$

\subsection{New platforms and enhancements}

Several flexible new platform classes were added to KNEE and EASEELib to support the code refactorization described in chapter 2. These are AcousticTransmitter, AcousticReceiver, SeismicTransmitter, SeismicReceiver, VisibleTransmitter, VisibleReceiver, RadioFreqTransmitter, and RadioFreqReceiver. The first six of these are in KNEE, whereas the last two are in EASEELib. As the names suggest, the platforms provide generic 
capabilities for transmitting and receiving signals. Also added was a platform called InferenceTransmitter, which produces inferences such as power levels or probabilities of detection directly, without modeling the signal emission and propagation process. This can be useful for debugging and some modeling applications.

Several new classes for ambient noise backgrounds were added. In KNEE, AcousticAmbientNoise and SeismicAmbientNoise provide non-sensitive ambient noise backgrounds for acoustics and seismics. (There is no VisibleAmbientNoise platform because the optical calculations do not use ambient noise backgrounds in the same sense.) Higher-resolution counterparts in EASEELib are: NoiseBackgroundAcoustic (acoustic noise), NoiseBackgroundInfrasonic (infrasonic noise), NoiseBackgroundSeismic (seismic noise), NoiseBackgroundVHFAndUHF (RF noise in the VHF and UHF ranges), and NoiseBackgroundSHFAndEHF (RF noise in the SHF and EHF frequency ranges).

Several of the platform classes in EASEELib that serve generic functions were extended to include RF and infrasonic capabilities. These include GenericTransmitter, which can be custom-configured to transmit any types of signals; PowerSensor, which can be custom-configured to measure power of any types of signals; and DetectionSensor, which can be custom-configured to calculate probabilities of detection and false alarm for any types of signals.

The Human platform class was rewritten as an extension of MultiPlatform, i.e., a platform that encapsulates multiple other platforms. In this case, the encapsulated platforms are HumanActivity, which has a signature for human footsteps; HumanAuditory, which models the human hearing response; and HumanVisual, which models the human visual response. This restructuring facilitates more flexible modeling of human signatures and sensing.

Some new signatures for motorcycles, a Polaris, and pick-up trucks recorded for the SPRUCE project were also added to EASEELib. A new class was created called GroundVehicleMotorcycle to incorporate the motorcycle signatures. The Polaris and pick-up truck signatures were added to the existing GroundVehicleWheeled class.

Lastly, a model for wireless local area network (WLAN) emissions in the super-high frequency (SHF) frequency range was added to the platform representing unmanned aircraft systems. 


\section{Environmental Representation}

As discussed in the Introduction, the primary objective of the SPRUCE project was to enable $3 \mathrm{D}$ signal propagation calculations using high-resolution environmental data. To this end, EASEE's environmental representation was substantially upgraded to incorporate more complex terrain data, 3D atmospheric structure, and 3D seismic (subsurface) structure. Those upgrades are the topic of this chapter. Section 5.1 begins with a general discussion of the environmental data abstraction layer and recent enhancements. Then, section 5.2 addresses a topic impacting the entire environmental representation, namely the handling of geographic coordinate transformations. Lastly, sections 5.3 and 5.4 describe upgrades specific to the terrain and weather representations, respectively.

\subsection{Environmental data abstraction layer}

As depicted in Figure 1, EASEE includes a self-contained representation, or data abstraction layer, for the environment. The environment conceptually includes terrain surface characteristics (elevations, landcover, and snow), subsurface (soil and seismic profiles), and atmospheric properties. EASEE's abstraction layer is one of the key features in the software framework, as it isolates the modeling capabilities from the data resources used to specify the environment. Whenever a new type of environmental data is interfaced with EASEE, it will become usable by all signal modeling capabilities implemented in EASEE. Conversely, whenever a new modeling capability is integrated with EASEE, it can be used with all types of environmental data recognized by the abstraction layer.

The abstraction layer is defined by the Java class EnvironScenario and its three non-abstract subclasses: EnvironHomo, which represents a homogeneous environment (constant atmosphere and subsurface); EnvironVertProf, which represents an environment with vertical variations (profiles) only; and EnvironThreeDim, which represents an environment with full $3 \mathrm{D}$ variations. The last of these is new to EASEE v3. Figure 12 shows the hierarchy of environmental scenarios and the elements comprising them. 
Figure 12. Hierarchy of the environmental scenarios in EASEE and the objects contained within each scenario. Blue font indicates additions made as part of the SPRUCE project.

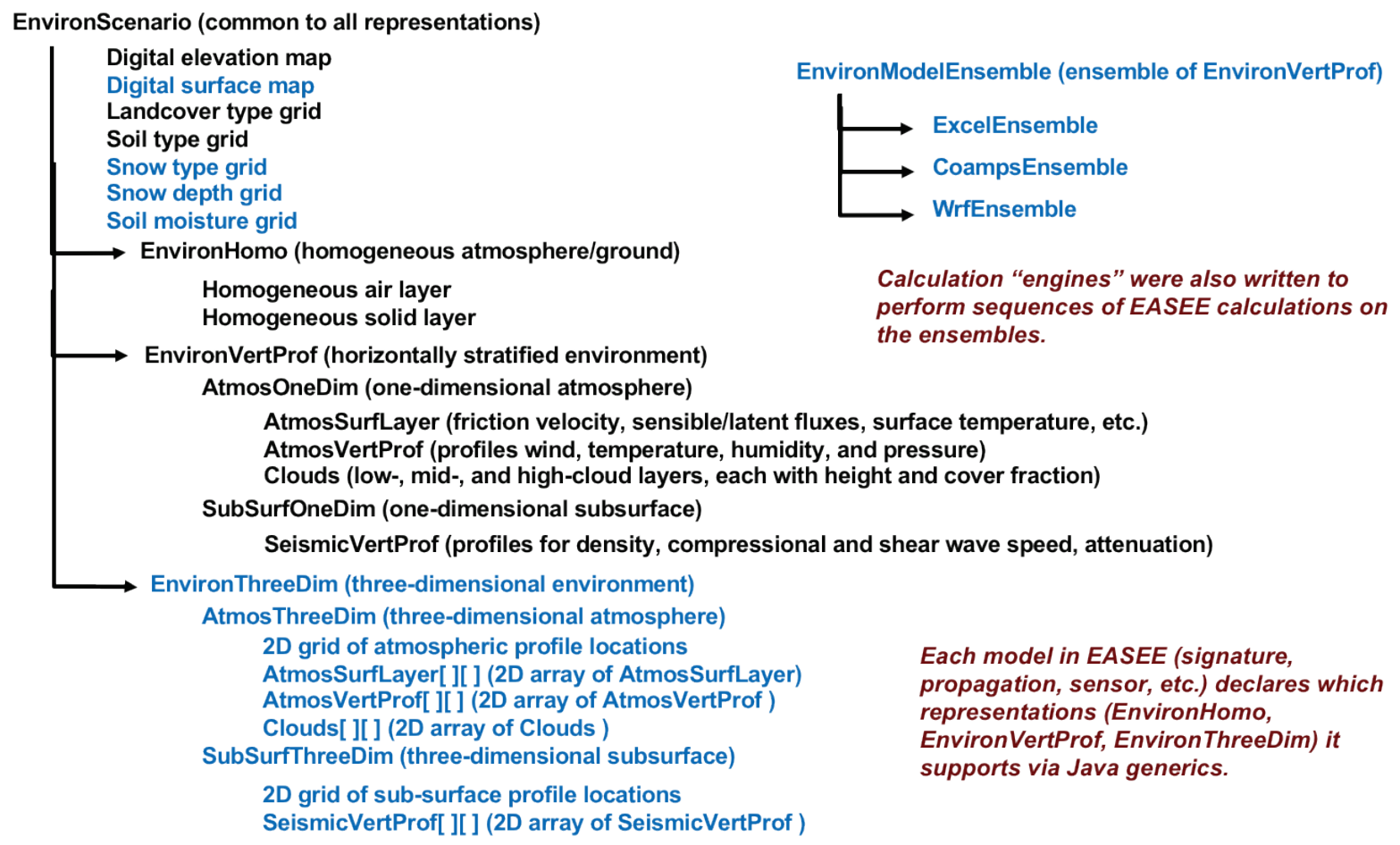

The hierarchy and its elements may be described as follows:

- EnvironScenario. This class includes a bare-Earth digital elevation map, DSM (for the height of elements on top of the terrain, such as buildings and trees), landcover type grid, soil type grid, soil moisture fraction grid, snow type grid, and snow depth grid. By grid, we mean an array of values describing how these properties vary in the horizontal coordinates, i.e., along the Earth surface according to the geographic projection. Since EnvironScenario is the parent class for EnvironHomo, EnvironVertProf, and EnvironThreeDim, those classes inherit all the data in EnvironScenario.

- EnvironHomo. This class adds a single air layer to represent the atmosphere and a single solid (isolinear) layer to represent the subsurface; that is, the atmosphere and subsurface are assumed to be homogeneous half spaces.

- EnvironVertProf. This class adds a 1D atmospheric representation (the AtmosOneDim class) and a 1D subsurface representation (the SubSurfOneDim class). These representations are $1 \mathrm{D}$ in the sense that only vertical variations are included, i.e., there is no variation in the horizontal directions lateral to the Earth's surface. The AtmosOneDim class 
in turn has three components: a surface layer (the AtmosSurfLayer class, which specifies a friction velocity, sensible/latent fluxes, surface temperature, and surface humidity), vertical atmospheric profiles (the AtmosVertProf class, with profiles wind, temperature, humidity, and pressure), and clouds (the Clouds class, which has low-, mid-, and high-cloud layers, each with an altitude and coverage fraction). The SubSurfOneDim class specifies vertical profiles consisting of solid, isolinear layers (the SolidIsoLinearLayers class, which specifies density, bulk, and shear modulus, from which the compressional and shear wave speed can be derived).

- EnvironThreeDim. This class is very similar to EnvironVertProf except that it contains objects of the AtmosThreeDim and SubSurfThreeDim classes. These are like their $1 \mathrm{D}$ counterparts (AtmosOneDim and SubSurfOneDim) except that they contain 2D arrays of AtmosSurfLayer, AtmosVertProf, Clouds, and SolidIsoLinearLayers objects. The arrays (grids) represent variations in the horizontal directions.

The non-abstract environmental scenario subclasses possess numerous Java constructors that enable the scenarios to be created from a great variety of environmental data resources. Wilson et al. (2019)* describe some of these constructors. A complete description can be found in the EASEE JavaDoc (Hypertext Markup Language [HTML] format Java Documentation).

\subsection{Geographic coordinate transformations}

To lay the groundwork for the incorporation of high-resolution atmospheric and terrain data into EASEE from a variety of data sources, components of the code handling geographic coordinate transformations were entirely revamped as part of EASEE v3.

Earlier versions of EASEE allowed users to load and manipulate environmental data specified in the geodetic (as opposed to geocentric) latitude and longitude and the Universal Transverse Mercator (UTM) systems. A dozen or so Earth ellipsoid (datum) models were supported. Regardless of the original coordinate system, the data were always converted to UTM coordinates on the WGS (World Geodetic System) 1984 ellipsoid for use in calculations.

\footnotetext{
* D. K. Wilson, R. E. Alter, K. M. Burch, and M. E. Swearingen. 2019. Using EASEE's Acoustical Calculations in Matlab. ERDC TR-19-1. Hanover, NH: ERDC-CRREL. https://doi.org/10.21079/11681/32009.
} 
In EASEE v3, a new abstract class was written called MapProjection, which supports data specifications in any geographic coordinate system and any Earth ellipsoid. Presently, non-abstract subclasses of MapProjection have been explicitly implemented for geodetic latitude and longitude, UTM, and the Lambert conical projections. The Lambert conical projection was added primarily to support ingestion of atmospheric fields from the Weather Research and Forecasting (WRF) weather model. Although more geographic projections could be added, at the present time there has not been a need.

Constructors for geographic coordinates and grids of coordinates now include an object of the type MapProjection. For example, the most general constructor for the GeoCoord class, which specifies a geographic coordinate in two dimensions, has three arguments: (1) the map projection to be used (a MapProjection object), (2) the x-coordinate in the projected coordinate system, and (3) the y-coordinate in the projected coordinate system. Specialized constructors are also available for the latitude and longitude, UTM, and Lambert systems. Cartesian grids of coordinates are constructed with the GeoGridCart2D class, which takes the map projection (among other options), the southwest corner of the grid (a GeoCoord object), the northeast corner of the grid (a GeoCoord object), and the number of points in the $\mathrm{x}$ - and $\mathrm{y}$-directions.

It is worth stressing here that the normal approach used by EASEE is to load and store environmental data in whatever geographic projection, and at whatever resolution, they were originally specified. The coordinate system (projection) and the resolution of the environmental data, as well as the coordinate system and resolution of any calculations performed with the data, are fully independent. For example, if a WRF run is performed on a Lambert conical projection at $3 \mathrm{~km}$ resolution, those data are generally loaded and stored by EASEE as is. They are not converted to the coordinate system and resolution of the calculation until the calculation is performed. Furthermore, different components of the environmental data may be specified with different coordinate systems and resolutions. For example, the atmospheric data, terrain elevations, and landcover could all have differing projections and resolutions. This approach avoids resampling of the environmental data until it is required, thus preserving the data in their original fidelity. 
Another significant upgrade in EASEE v3 is that signal propagation calculations are performed on a UTM projection with a domain (coordinate grid) that is set dynamically when the propagation algorithm runs. Previously, the calculation domain was set when the propagator was constructed. However, the propagators had to be reconstructed each time the domain was changed, which can be very time consuming for computationally intensive propagation algorithms. It is also important to understand that the grid specified for the calculation is what drives the resolution of the calculation, rather than the resolution of the grid for the environmental data. For example, if 1-cm resolution terrain elevation data are loaded into EASEE, but a 1-m resolution domain is specified for an LOS calculation, the calculation will be performed at $1-\mathrm{m}$ resolution by down sampling the resolution of the specified terrain elevations.

\subsection{Terrain representation and inference of terrain properties}

Before EASEE v3, the terrain surface representation included the DTM, landcover type, and soil type. EASEE v3 expanded this representation to include the DSM, soil moisture, snow type, and snow depth. The DTM represents the altitude of the bare Earth, typically specified relative to an Earth ellipsoid. The DSM represents the height of objects, such as trees and buildings, on top of the bare Earth. One of the primary motivations for adding the DSM to EASEE is to support probabilistic line-of-sight (PLOS) calculations through vegetation, as will be discussed in section 6.3.

Support for many additional standardized data landcover formats was also added to EASEE v3. The formats now supported are NLCD (National Landcover Dataset) 1992 and 2001; GeoCover; VisNav; and the FASST (Fast All-season Soil STrength, Frankenstein and Koenig 2004) vegetation types. The soil types are also based on the FASST scheme, which is itself a generalization of the Unified Soil Classification System (USCS). The snow types, based on unpublished recommendations by former CRREL researcher D. Albert, consist of "Snow, fresh," "Snow, mature always cold," "Snow, mature near freezing," "Snow, melting," and "Snow, patchy."

All the terrain elements mentioned in the preceding paragraph are represented as geographic rasters; that is, they are $2 \mathrm{D}$ arrays that describe spatial variations on a geographic coordinate grid. If only a single value is specified for any of these elements, it is assumed to be constant everywhere. 
Another significant improvement in EASEE v3 is the development of an extensive scheme for inferring unspecified terrain properties; for example, if the user provides terrain elevation and landcover data but not surface elevation and soil data, reasonable values will be inferred for the surface elevations and soil based on the terrain elevation and landcover. This is an important capability because it enables EASEE to run and produce meaningful results when users do not have comprehensive geospatial data available for a given site of interest. Figure 13 shows the flowchart inferring unspecified terrain properties. The basic process is:

- If the user has no landcover (LC) data, a single LC type will be specified for the entire domain. A single soil type will be inferred from the LC, or a single soil type will be specified by the user for the entire domain.

- If the user has LC data but no soil data, either the soil type will be inferred from the LC, or a single soil type will be specified by the user for the entire domain.

- If the user has no DTM, flat Earth will be assumed, and the DSM will be inferred based on the landcover.

- If the user does have a DTM but no DSM, the DSM will be inferred from the DTM and landcover.

Figure 13. Flowchart for inferring unspecified terrain properties from the specified ones.

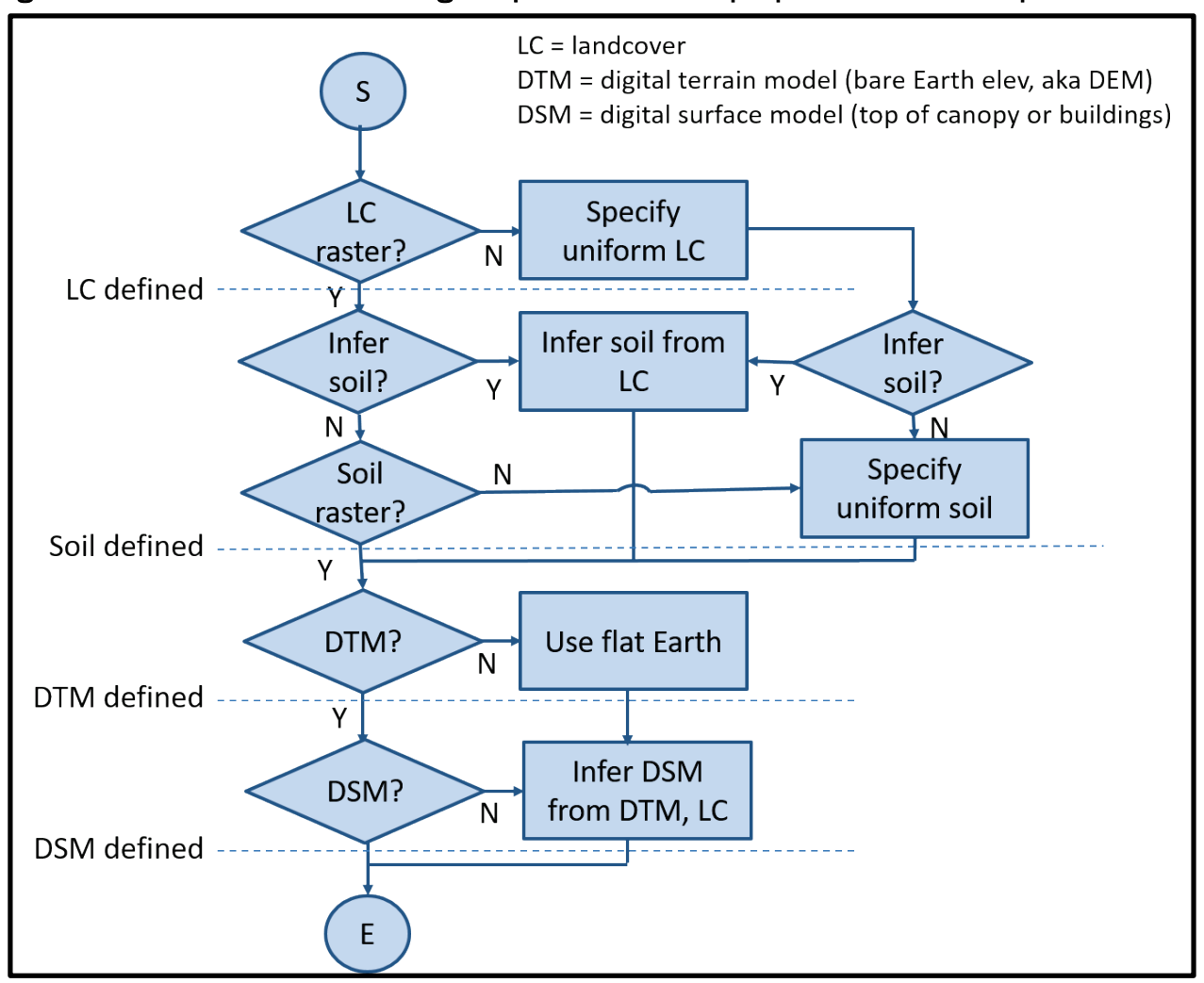


As mentioned in section 3.1, EASEE is often distributed with a Matlabbased interface, called the MatlabGUI. For the SPRUCE project, the MatlabGUI was updated to include snow type, snow depth, and soil moisture. For simplicity, the present version supports only the NLCD2001 and Geocover landcover specifications, and the soil/subsurface properties are always inferred from these. Previous versions supported the FASST vegetation and soil types, but this was removed. The primary reason is that allowing an untrained user to separately specify soil and vegetation types can lead to modeling inconsistencies, e.g., when the vegetation type is set to coniferous forest and the soil type is set to asphalt. Additionally, some of the landcover schemes include a category for permanent ice/snow, which can lead to some confusion when there is a separate specification of the snow type and depth. The snow type and depth specified in the GUI is interpreted as a dynamic snow cover on top of the permanent ice/snow.

\subsection{Atmospheric representation}

For EASEE v3, two new Java classes, AtmosOneDim and AtmosThreeDim, were created that support $1 \mathrm{D}$ and $3 \mathrm{D}$ specification of atmospheric structure, respectively. The EnvironVertProf class contains an AtmosOneDim object, whereas the EnvironThreeDim class contains an AtmosThreeDim object. (See Figure 12.)

AtmosOneDim has three elements: (1) an atmospheric vertical profile object (with vector wind, temperature, specific humidity, and pressure as a function of height), (2) an atmospheric surface layer object (with friction velocity, sensible heat flux, and latent heat flux), and (3) a cloud (with low-, mid- and high-altitude cloud fractions) object. These objects are little changed from previous versions of EASEE. Wilson et al. (2019)* provides a useful description of their properties. AtmosThreeDim is very similar to AtmosOneDim, except that it includes a geographic coordinate grid and 2D arrays of the vertical profiles, the surface layer, and the clouds. Each of the $2 \mathrm{D}$ arrays describes the variation of these entities across the specified geographic coordinate grid.

Support for initializing atmospheric objects from several numerical weather forecast models was added, namely (1) the WRF model, which is the primary forecast model used by the U.S. weather research community

\footnotetext{
* D. K. Wilson, R. E. Alter, K. M. Burch, and M. E. Swearingen. 2019. Using EASEE's Acoustical Calculations in Matlab. ERDC TR-19-1. Hanover, NH: ERDC-CRREL. https://doi.org/10.21079/11681/32009.
} 
and the U.S. Army, (2) the Coupled Ocean/Atmosphere Mesoscale Prediction System (COAMPS), which is the U.S. Navy's primary forecast model, and (3) the Global Air-Land Weather Exploitation Model (GALWEM), which is a version of the U.K. Meteorology Office forecast model that was modified to meet the needs of the U.S. Air Force, which recently adopted it as their official mesoscale meteorology model. GALWEM is also now being used for Army operational weather support. With COAMPS, WRF, and GALWEM, EASEE now supports all the major weather forecast models used by DoD.

To load WRF and COAMPS forecasts, Java classes were written that initialize AtmosOneDim and AtmosThreeDim objects, respectively. Specifically, the WrfLoader class is an extension of AtmosOneDim, which loads WRF data from files with Network Common Data Format (NetCDF) format and processes them into the profiles as described above. The loader handles conversions between the geopotential height (the vertical coordinate used by the forecast model) and the actual height above ground level (as needed for the acoustical calculations). Similarly, WrfLoader3D is an extension of AtmosThreeDim and loads WRF data from NetCDF-format files into a full $3 \mathrm{D}$ atmospheric representation. WrfLoader3D was written specifically for this project to enable $3 \mathrm{D}$ infrasound propagation calculations. The classes CoampsLoader and CoampsLoader3D analogously extend AtmosOneDim and AtmosThreeDim. At present, only a 1D loader, GalwemLoader, has been written to load data from GALWEM.

When the classes based on AtmosOneDim are used to load profiles, a single geographic coordinate is specified, and the profiles nearest to this location are loaded. For the classes based on AtmosThreeDim, a pair of geographic coordinates are specified that indicate the southwest and northeast corners of the domain of interest, and the profiles within this entire domain are loaded.

An example infrasound propagation calculation made with EASEE based on $3 \mathrm{D}$ WRF data can be found in Wilson et al. (2018). The calculation demonstrates the importance of $3 \mathrm{D}$ propagation effects in a region with heterogeneous surface properties, namely a region spanning the Chesapeake Bay and nearby land. 


\subsection{Subsurface representation}

Analogously to the atmospheric representation, EASEE v3 defines two new Java classes, SubSurfOneDim and SubSurfThreeDim, which describe 1- and $3 \mathrm{D}$ subsurface (seismic) structure. Objects of these classes are incorporated by EnvironVertProf and EnvironThreeDim.

SubSurfOneDim contains a SolidIsoLinearLayers object, which consists of an array of profile transition depths and an array of SolidIsoLinear objects. Between each of the specified transition depths, a SolidIsoLinear object represents a solid, isotropic, linear, lossless medium. SubSurfThreeDim generalizes the $1 \mathrm{D}$ representation to include a geographic grid and a $2 \mathrm{D}$ array of SolidIsoLinearLayers, which describe the seismic profiles at each location on the geographic grid. 


\section{Signal Propagation and Noise Modeling}

As discussed in section 5.1, prior versions of EASEE supported just two models for the environment. One (EnvironHomo) assumes that the atmosphere and subsurface are homogeneous media. The other (EnvironVertProf) assumes that the atmospheric and subsurface properties vary only in height (depth). Calculations of signal propagation were subsequently based on one of these two models. Most of the calculations also assumed that the terrain properties, such as landcover and elevation, were homogeneous. Given these assumptions, the propagation exhibits certain symmetries that can be exploited to improve the efficiency of calculations. For example, the calculation may be independent of the azimuthal angles. The symmetry can be exploited by first performing a calculation over flat ground and adding terrain diffraction as a post-processing step. From a computational standpoint, this makes sense because with methods, such as a $\mathrm{PE}$, the initial calculation is very expensive, while the additional terrain diffraction calculation is relatively fast.

This chapter describes extensions inherent to EASEE v3 related to modeling signal propagation in complex environments. By complex, we mean environments such as urban, mountainous, and forested terrain, where significant wave scattering, diffraction, and multipath effects are present. In such situations, simplifying symmetries are often not available, and the implementation of the propagation calculations thus becomes considerably more complicated. Numerical methods must typically incorporate $3 \mathrm{D}$ spatial variability in the environment.

In addition to signal propagation, environmental background noise also plays a very important role in sensor performance calculations. In particular, the noise determines the false-alarm rate in probability of detection calculations. In EASEE, the background noise is modeled with a platform object; such objects were discussed in chapter 4 . This chapter also describes some of the updates to the acoustic and RF noise models.

\subsection{Propagator, parameter, and media classes}

One of the major tasks in upgrading EASEE to support $3 \mathrm{D}$ signal propagation calculations involved changes to the parameters classes supporting the propagation calculations. In earlier versions of EASEE, each signal propagator (i.e., Java class encoding a signal propagation algorithm) had 
an associated parameters class, the purpose of which was to define the parameters needed to perform the calculation. The parameters could include computational settings (e.g., options for including certain weather and terrain effects, the resolution of the calculation, and the attenuation in the numerical sponge layer at the boundaries of the calculation), as well as information on the atmospheric and terrain environment needed for the calculation. Each parameters class was required to include a method called setEnviron, which converted environmental scenarios (objects representing the full atmosphere, terrain, and subsurface environment) to the environmental information needed for the propagation calculation. Many of the parameters classes also provided alternative methods for specifying the environmental information as needed by a particular algorithm. These alternative methods were mainly intended for running the propagation calculation outside of the full EASEE gridded calculation framework.

Limitations of this approach became apparent during efforts to extend the propagation calculations to more complex environmental representations and to support arbitrary map projections. The essential problem is it is natural to set the computational parameters for the propagator when it is first constructed, whereas the environmental parameters are naturally set dynamically (at runtime), when the user has specified the projection and grid for the calculation, and when the environmental properties on this grid can be inferred from the atmospheric, terrain, and subsurface data resources. Previous versions of EASEE had to construct the environmental representation for the propagation calculation before the location of the calculation was specified. To circumvent this problem, nearly all available environmental information was stored in the parameters class, extracted, and converted to what was needed for the calculation at runtime. This approach is inefficient, particularly when the environmental data are very large, as is the case for a full $3 \mathrm{D}$ calculation.

EASEE v3 makes two primary changes to the propagator classes, which may initially seem subtle but greatly improve the software functionality. These are: (1) the map projection and the grid for the calculation are set dynamically at runtime, rather than when the propagator is constructed, and (2) the previous parameters classes were partitioned into separate classes for the computational parameters and for the environmental properties. These are now referred to as the "parameter" and "media" classes, respectively. The parameters object is always constructed at the same time the 
propagator is constructed, although they may be changed afterwards if desired. The media object may be specified when the propagator is constructed or later within the flow of a full EASEE calculation.

The media classes now contain only the information that is strictly necessary for the associated propagation calculations to run. The setEnviron method was moved from the parameters class to the propagator itself. It is now invoked at runtime, during the execution of the full EASEE gridded calculation. It is not used when the propagation calculation is run outside the context of a full EASEE calculation. Figure 14 shows the basic flow of information from the environmental data, to the environmental scenario objects, to the media objects, and finally to the propagators.

Media specifications were written to support "homogeneous," "polar," and "Cartesian" specifications of the environmental properties. "Homogeneous" means that the environmental properties do not vary regarding the horizontal coordinate, although they may still vary vertically. This representation is essentially the legacy EASEE v2 approach. "Polar" means that the environmental properties vary in three dimensions and are specified on an azimuth/range grid relative to a known geographic origin. "Cartesian" also means that the environmental properties vary in three dimensions, except they are specified on a regular rectangular grid. Propagation models, depending on how they are formulated, support one or more of these three specifications. The supported specifications are indicated using Java generics to ensure that the algorithm is applied only to the geometry of environmental data for which it is intended.

Figure 14. The EASEE environmental abstraction layer (EnvironScenario and its subclasses) is constructed from a variety of data resources for terrain elevation, landcover, soils, and atmospheric forecasts and models. The environmental data are converted to the propagator media, which are then used for signal propagation modeling.

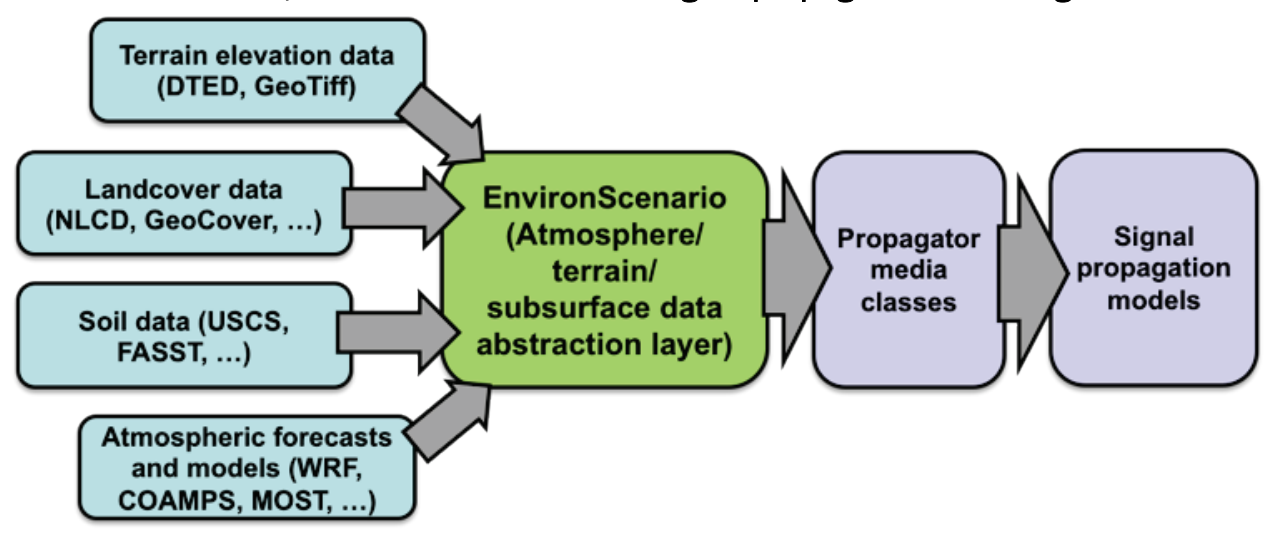


The two basic ways in which propagation calculations can be run are

1. As a standalone propagation calculation:

a. Construct the propagator parameters object with the desired numerical settings for the calculation.

b. Construct the propagator media object describing the atmospheric, terrain, and subsurface properties (as needed) for the calculation.

c. Construct the propagator with the parameters and media objects from Steps 1 and 2.

d. Set up a grid for the calculation. The grid can be specified as an EASEE geographic grid (a Cartesian grid based on a map projection), a structured grid, or an unstructured grid.

e. Call the propagator's calcTransGridGeo, calcTransGridStruct, or calcTransGridUnstruct method to perform the calculation (for a geographic grid, structured grid, or unstructured grid, respectively).

2. Within the full EASEE gridded calculation framework (Figure 15):

a. Construct the propagator parameters object with the desired numerical settings for the calculation.

b. Construct the propagator with the parameters object from Step 1.

c. Attach the propagator from Step 3 to the propagation services for the calculation. (The propagation services collect together the propagators for all signal modalities.)

d. Set up a geographic grid for the calculation. Within the context of a full EASEE calculation, this is typically done through a GUI in which the user selects an area of interest (AOI) and resolution.

e. Specify the environmental data (atmosphere, terrain, subsurface) for the calculation. This is typically done through a GUI in which the user specifies the data resources to be used (e.g., weather forecasts and terrain elevation data). 
f. Invoke an EASEE gridded calculation using one of EASEE's many different classes for this purpose (GriddedCalculationsEns2D, GriddedCalculationsTime2D, etc.). These classes invoke the calculationEngine method in GriddedCalculations, which performs the steps necessary for a full EASEE calculation.

g. The calculationEngine method will configure the propagation services using the map projection for the geographic grid in Step 4 and the environmental data from Step 5. This is done using the setMapProj and setEnviron methods of the propagators.

h. The calculationEngine method links together the various platforms involved in the calculation and calls the propagateSignals methods as needed to complete the calculation.

i. When the calculation completes, calculationEngine returns the inferences (e.g., probability of detection) on the geographic grid specified in Step 4.

Figure 15. Process by which propagation calculations are run in EASEE. The propagator (computational) parameters are constructed and then used to construct the propagator, which encapsulates the actual signal propagation model. The environmental representation is used to construct the propagator media. The media, calculation grid, and signature are dynamically sent to the propagator, which performs its task and sends the output to the sensor model.

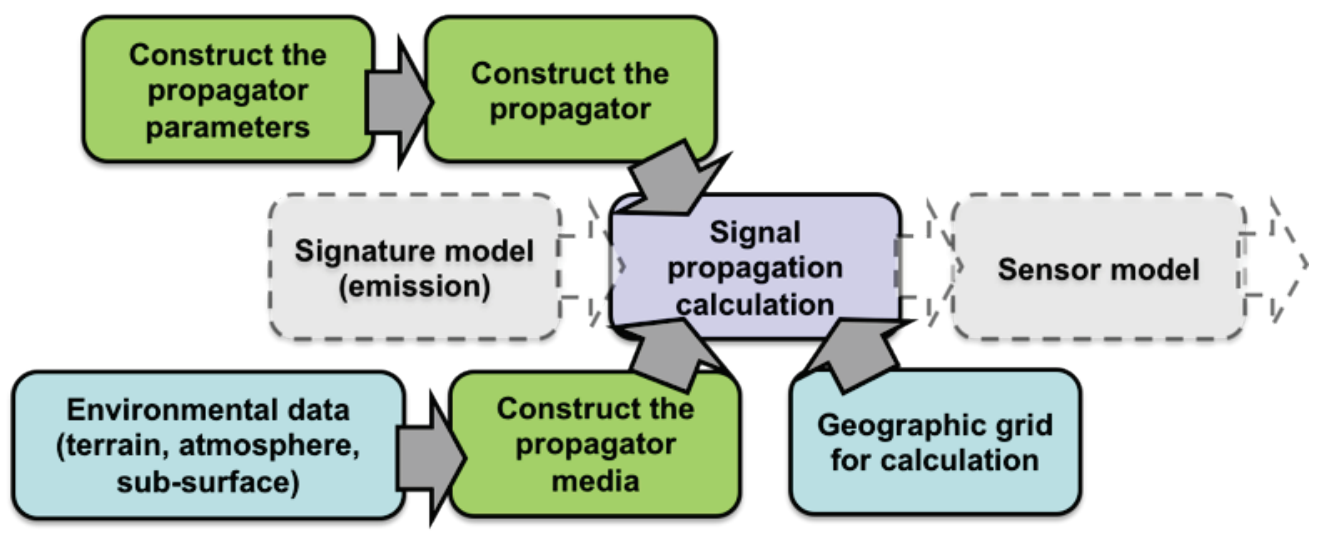

\subsection{Propagator class hierarchies}

The transition to EASEE v3 and additions for various other projects in recent years resulted in extensive changes in the propagator classes. This section summarizes the current structure of these classes. 
The abstract class serving as the parent class for all propagators is called BasePropagator. Similarly, BasePropagatorParams is the base class for all the propagator parameters classes, and BasePropagatorMedia is the base class for all the propagator media classes.

Several classes, both abstract and non-abstract, extend BasePropagator. The direct extensions are shown on the left side of Figure 16. All the classes shown are non-abstract, except for BaseCoherencePropagator and BaseStructGridPropagator. BaseCoherencePropagator is the parent class for propagators performing coherence calculations, and BaseStructGridPropagator is the parent class for propagators performing calculations on structured grids, as will be described shortly. The other, non-abstract classes extending BasePropagator perform specialized functions (such as ReplicatorPropagator, which simply copies data values across the domain for noise backgrounds, and GeomSpreadPropagator, which performs generic geometrical spreading calculations with attenuation) or perform calculations in complex terrain for which no symmetry assumptions are possible (e.g., LineOfSightPropagator, which performs LOS calculations, and HataModelPropagator, which performs RF propagation calculations in heterogeneous terrain).

Also shown in Figure 16, on the right, is the abstract class BaseStructGridPropagator and its extensions. The structured-grid calculations, which were discussed in Wilson and Yamamoto (2014), use symmetries in certain propagation problems to improve computational efficiency. An example is the Crank-Nicholson PE calculation (CNParabolicEqnPolar and CNParabolicEqnCart), which depends only on the height of the source, height of the receiver, horizontal distance between the source and receiver, and the direction relative to the wind. Hence, once a calculation has been performed, it can be "reused" for different source locations on the terrain. Some important features of the BaseStructGridPropagator class include an optional post-processing stage to include terrain diffraction and automatic saving and loading of calculations to file so they are not repeated.

Figure 17 left shows BasePropagatorParams and some of its subclasses, whereas the right side of the figure shows BasePropagatorMedia and some of its subclasses. BasePropagatorParams is an empty class, which simply serves as the parent class for the other parameters' classes. The parameters are typically highly specific to the propagation calculation, as will be discussed in some of the examples of acoustic and RF propagators later in this section. 
Figure 16. Left: Parent class for the propagators (BasePropagator) and its direct subclasses. Right: Parent class for the structured-grid propagators (BaseStructGridPropagator) and its direct subclasses.

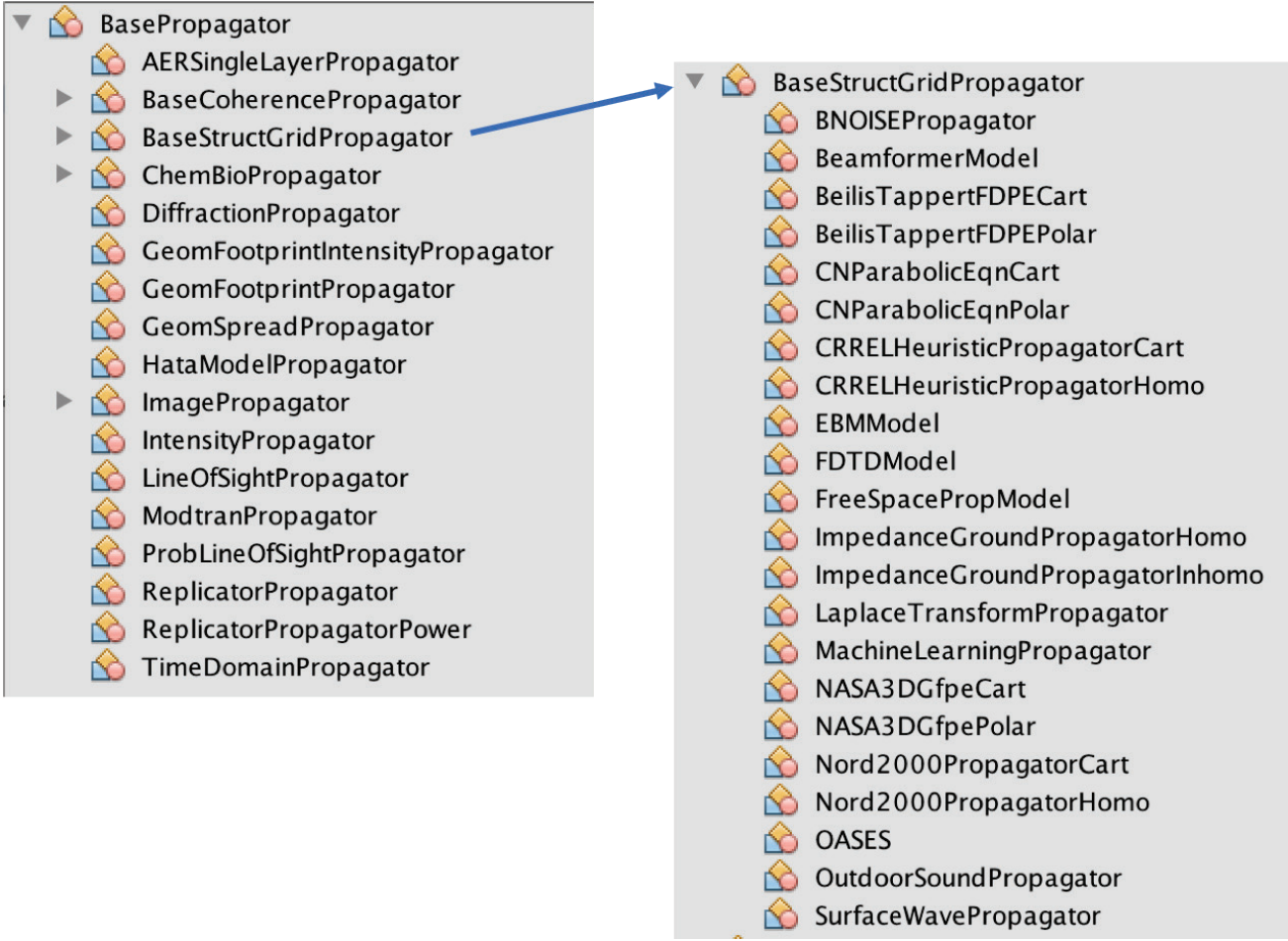

Figure 17. Left: Base class for propagator parameters (BasePropagatorParams) and some of its subclasses. Right: Base class for propagator media (BasePropagatorMedia) and some of its subclasses.

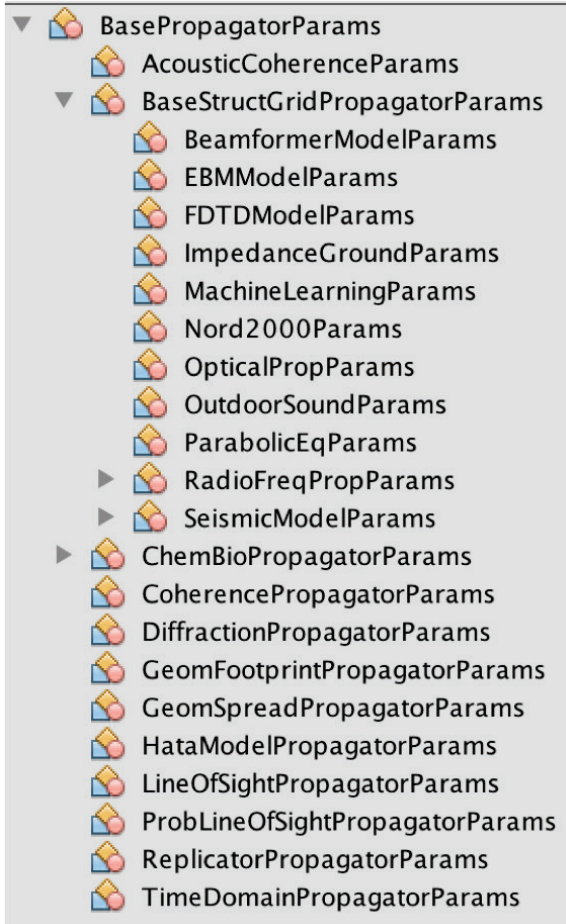

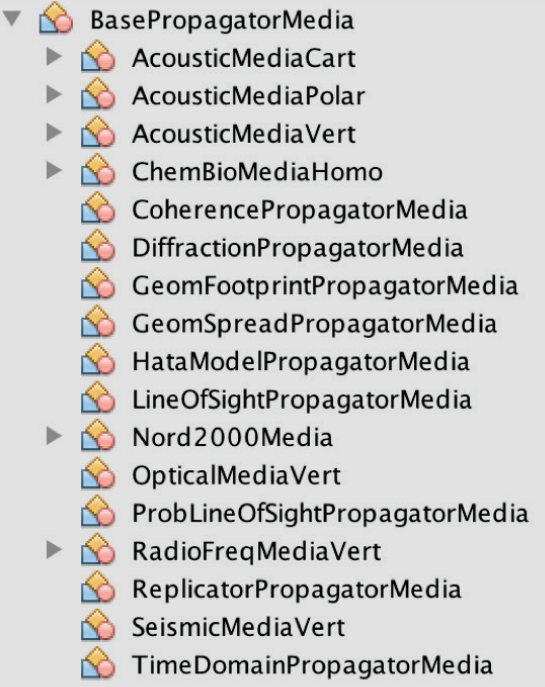


BasePropagatorMedia includes only a digital elevation map (DEM), which represents the height of the bare Earth relative to sea level within the AOI. All other environmental information is stored in media classes on an asneeded basis for the propagators. For example, LineOfSightPropagatorMedia additionally incorporates a DSM, which is useful for the LOS calculations. The acoustic media classes, which will be discussed in more detail in section 6.5.2, incorporate additional environmental information impacting sound propagation, such as atmospheric wind and temperature, and ground-surface properties.

\subsection{PLOS calculations}

In many situations of practical interest, landcover (vegetation and buildings) may partially obstruct LOS. Often, the landcover is too small or dynamic to be resolved in a deterministic sense, making it natural to assess the probability that the LOS is obstructed. For example, one cannot generally predict whether individual leaves on a tree will block LOS; rather, one can predict only the probability that the leaves, taken as a whole, will block the LOS. We call such a calculation a PLOS model.

PLOS calculations are a new capability in EASEE v3. The calculations are fully $3 \mathrm{D}$ in the sense that they account for both vertical and horizontal variability in the vegetation, arbitrary viewer and target locations. The probability is assumed to attenuate exponentially with distance; that is, the probability of seeing through a homogeneous layer of vegetation is

$$
P=e^{-\alpha d}
$$

where $d$ is the length of the path, and $\alpha$ is an extinction rate for the probability, which depends on the density and arrangement of the vegetation.

We can calculate the probability of seeing through two layers, $P_{12}$, by noting this equals the probability of seeing through the first layer, $P_{1}$, times the probability of seeing through the second layer, $P_{2}$. (This relationship assumes the ability to see through each of the two layers is independent). Thus,

$$
P_{12}=P_{1} P_{2}=e^{-\alpha_{1} d_{1}-\alpha_{2} d_{2}},
$$

where $d_{1}$ and $d_{2}$ are the path lengths in layers 1 and 2, and $\alpha_{1}$ and $\alpha_{2}$ are the extinction rates in layers 1 and 2. 
The PLOS calculation in EASEE is based on extending this approach to a three-layer medium, as shown in Figure 18. The three layers are associated with open air (which does not block the LOS), a canopy layer, and a subcanopy layer. The canopy and subcanopy layers have their own thicknesses $\left(h_{c}\right.$ and $h_{s}$, respectively) and extinction coefficients $\left(\alpha_{c}\right.$ and $\alpha_{s}$, respectively). The names for these layers are somewhat arbitrary; the important aspect of the model is that it accommodates two distinct layers in the landcover. If only a single-layer model is desired, the thickness of one of the layers in the two-layer model can be set to zero. More than two layers could readily be accommodated in a future version if desired.

Figure 18. Geometry of the PLOS calculation. Here $d_{a}$ is the path length in the air layer, $d_{c}$ is the path length in the canopy layer, and $d_{s}$ is the path length in the subcanopy layer.

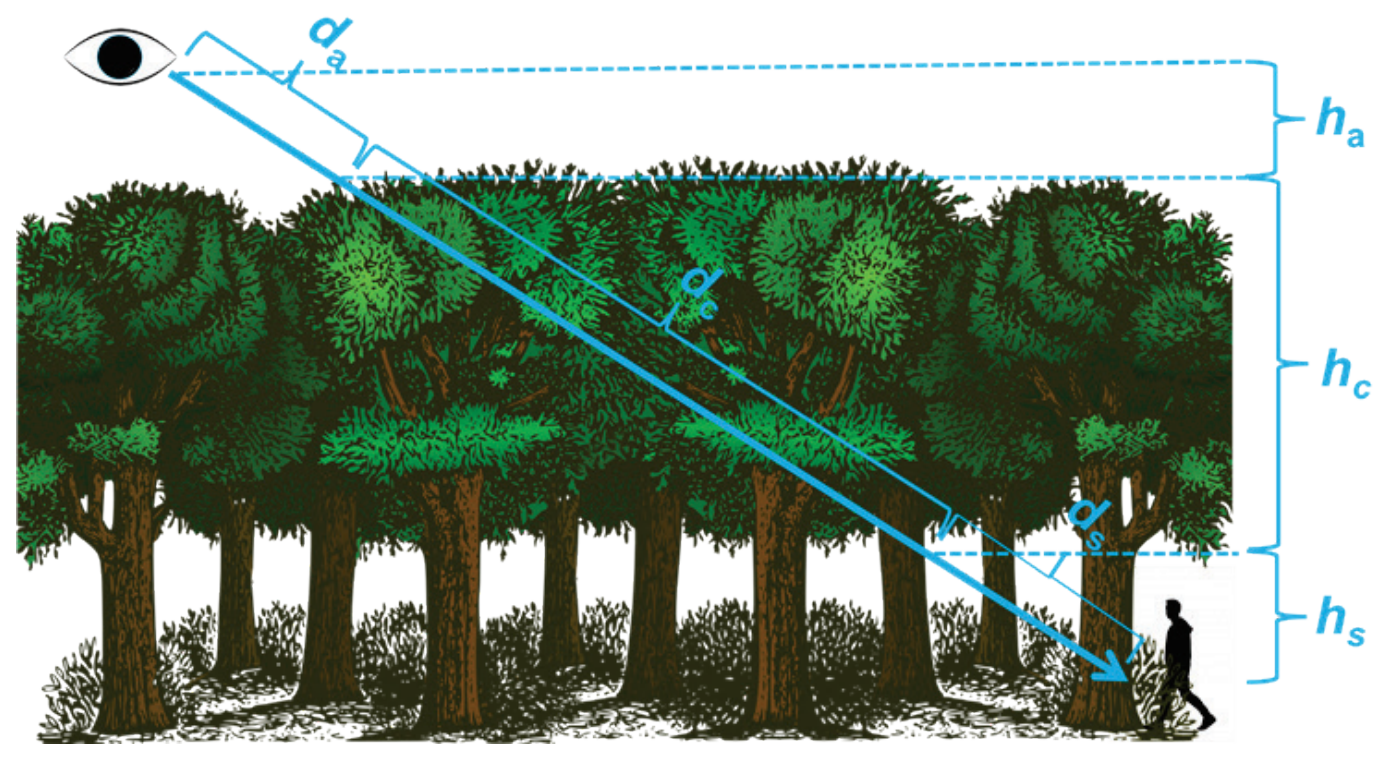

Note that the paths through the layers shown in Figure 18 may be at a slanted angle through the vegetation. The path lengths through the layers, $d_{c}$ and $d_{s}$, are related to the horizonal distances traveled, as $r_{c}$ and $r_{s}$, through the relationship $r_{c}=d_{c} \cos \theta$, etc., where $\theta$ is the slant angle of the path relative to the horizontal (generally called the elevation angle). Furthermore, the layer thicknesses are given by $h_{c}=d_{c} \sin \theta$, etc. Hence, the probability of seeing the target based on Figure 18 is

$$
P=e^{-\left(\alpha_{c} r_{c}+\alpha_{s} r_{s}\right) / \cos \theta}=e^{-\left(\alpha_{c} h_{c}+\alpha_{s} h_{s}\right) / \sin \theta}
$$

In practice, the PLOS calculations are implemented on a $2 \mathrm{D}$ grid (raster) of landcover properties. Therefore, it is necessary to calculate $r_{c}$ and $r_{s}$ 
within each grid cell $(i, j)$, where the $i$ and $j$ are indices for the horizontal coordinates (typically the easting and northing, respectively). The LOS probability is then calculated from

$$
P=\sum_{i} \sum_{j} e^{-\left(\alpha_{c, i j} r_{c, i j}+\alpha_{s, i} r_{s, i j}\right) / \cos \theta},
$$

where $\alpha_{c, i j}$ is the canopy extinction coefficient in grid cell $(i, j)$, etc.

Another complication in the PLOS calculation is the variation in the bareEarth terrain elevation. If the path intersects with the bare Earth at any point, we set $P=0$.

The values for $h_{c}, h_{s}, a_{c}$, and $\alpha_{s}$ are clearly key to calculating a realistic LOS probability. However, these values are subject to substantial variability and uncertainty. At this present time, EASEE infers the values based on the landcover type. For example, for the GeoCover Evergreen Forest class, $h_{c}$ is set to $1.0 \mathrm{~m}, h_{s}$ to $5.0 \mathrm{~m}, a_{c}$ to $0.1 \mathrm{~m}^{-1}$, and $a_{s}$ to $0.02 \mathrm{~m}^{-1}$. The extinction values would imply that one can see about $10 \mathrm{~m}$ through the canopy and $50 \mathrm{~m}$ through the subcanopy. In the present version of EASEE, these values are only rough guesses. For programmatic reasons, the PLOS model development was terminated before a more systematic method (e.g., from previously published papers or from Light Detection and Ranging [LiDAR] point cloud data) to determine these values could be implemented. However, these values can be readily updated in the future since the core functionality for the PLOS calculations is fully operational.

\subsection{Diffraction model}

As mentioned in section 6.2, the BaseStructGridPropagator class, which implements calculations on structured grids (i.e., grids possessing some sort of symmetry), includes an optional post-processing stage to calculate terrain diffraction. Propagators that are not subclasses of BaseStructGridPropagator can also be programmed to use this terrain diffraction calculation if desired. It is based on approximating the terrain between the source and receiver with a wedge, as described in Wilson (1998). New to EASEE v3 is an option for setting the range and height of the "apex" (peak) of the wedge based on Bullington's (1977) method. The true Bullington method involves calculating the diffraction with a knife-edge shape (which is also an option available in EASEE v3), but the wedge approximation 
should yield more accurate results in gradually sloping terrain. The Bullington-based wedge-diffraction model is now the default in EASEE.

\subsection{Acoustic modeling enhancements}

From the outset, one of the strengths of EASEE has been its extensive acoustical modeling capabilities, which build on a legacy of Army-funded research over the past several decades. EASEE v3 provides a framework capable of supporting recent advances in modeling acoustic signal propagation in complex environments.

In this subsection, we first describe the revamping of the acoustical feature sets, which was done primarily to support infrasound calculations. We then describe how the environmental properties are modeled for acoustic calculations in EASEE v3. Then, we detail several of the propagation models that have been integrated. Lastly, we discuss some new additions to the nosier backgrounds.

\subsubsection{Feature definitions}

As discussed in Wilson et al. (2009) and Wilson and Yamamoto (2014), EASEE calculations are feature driven, in the sense that calculations are requested for particular signal features (characteristics) and serviced by the sequence of modules shown in Figure 1. Hence feature definitions play a critical role in the software design.

Starting with EASEE v3, the acoustic feature space was partitioned into three primary families of spectral features, as represented by Java interfaces. Each acoustic modeling capability in EASEE declares which of these feature families it supports. In principle, one, two, or all three families may be supported. The Java generics technique described in section 1.1 ensures that only capabilities applicable to the same feature families are interfaced. For example, an infrasonic sensor can be employed in a modeling sequence with an infrasonic signature, whereas a sensor applicable only to the audible range cannot be.

Figure 19 shows the Java classes that define the feature sets and their implementations. As currently implemented, "acoustic" spectral features span the frequency range including one-third octave bands from $6.3 \mathrm{~Hz}$ to $20 \mathrm{kHz}$. 
Figure 19. Acoustic and infrasonic feature classes in EASEE. There are three broad feature sets (families), which are coded as Java interfaces: AcousticSpectralFeature $(6.3 \mathrm{~Hz}$ to $20 \mathrm{kHz}$ ), AudibleSpectralFeature ( $20 \mathrm{~Hz}$ to $20 \mathrm{kHz}$ ), and InfrasonicSpectralFeature (1 Hz to $16 \mathrm{~Hz}$ ). Within each of these families, Java enumeration classes define specific features.

$\nabla \stackrel{0}{*}$ AcousticBaseSpectralFeature

$\nabla \stackrel{0}{\nabla}$ AcousticSpectralFeature

AcousticCoarseFeature

AcousticConfigurableFeature

AcousticFractionalOctaveFeature

AcousticOctaveFeature

AcousticOneThirdOctaveFeature

$\nabla \circ=0$ AudibleSpectralFeature

AudibleOctaveFeature

AudibleOneThirdOctaveFeature

$\nabla$ o $=0$ InfrasonicSpectralFeature

(B) InfrasonicOctaveFeature

(8) InfrasonicOneThirdOctaveFeature

This feature family is intended for general battlefield acoustics applications. It includes classes for pre-defined (enumerated) octave band features, one-third octave band features, one-twelfth octave band features, and for defining customized features with arbitrary center frequencies and bandwidths. The "audible" spectral feature family spans one-third octave bands from $20 \mathrm{~Hz}$ to $20 \mathrm{kHz}$. Pre-defined features for octave and one-third octave bands are provided. The "infrasonic" spectral feature family spans one-third octave bands from $1 \mathrm{~Hz}$ to $16 \mathrm{kHz}$. Pre-defined features for octave and one-third octave bands are provided. Note that the "acoustic" family overlaps with "audible" and "infrasonic," although the latter two are non-overlapping. To perform a calculation across the entire acoustic/infrasonic spectrum, one can thus perform separate calculations with "audible" and "infrasonic" feature sets, and then fuse them. Alternatively, one can perform a calculation with the "acoustic" feature set and merge it with an infrasonic calculation spanning one-third octaves from $1 \mathrm{~Hz}$ to $5 \mathrm{~Hz}$.

\subsubsection{Media representation}

As discussed in section 6.1, the media representations are a translation of the environmental representation, as appropriate to a particular signal modality and propagation model. For example, one component of the environmental representation is the physical properties of the soil, such as 
the permeability and porosity. For acoustical calculations, the values of these properties are not of direct interest, but we wish to know the acoustic impedance of the ground, which depends on those properties. Properties such as the acoustic impedance are, thus, calculated when the media representation is constructed from the environmental representation.

As shown in Figure 17, media representations for acoustic calculations involve three subclasses, AcousticMediaVert, AcousticMediaPolar, and AcousticMediaCart. These are all extensions of BasePropagatorMedia, the parent class for all types of propagator media. The acoustic media subclasses are appropriate for different geometries of propagation models. Specifically, AcousticMediaVert is for propagation algorithms capable of performing calculations only with homogeneous half spaces or vertical profiles; AcousticMediaPolar is for propagation algorithms capable of $3 \mathrm{D}$ calculations performed in polar coordinates; and AcousticMediaCart is for propagation algorithms capable of $3 \mathrm{D}$ calculations performed in Cartesian coordinates. Figure 20 shows the hierarchy of media classes and their components.

Figure 20. Acoustic media classes in EASEE. These classes represent the acoustical properties of the environment, as appropriate to signal propagation models with varying characteristics.

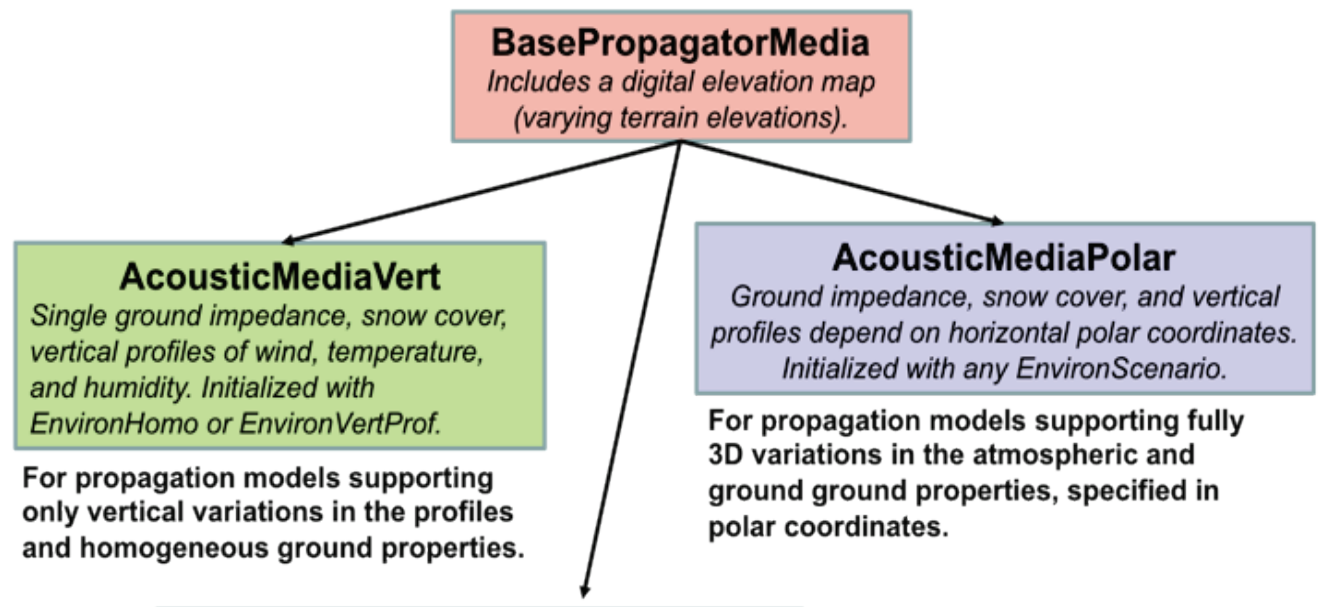

AcousticMediaCart
Ground impedance, snow cover, and vertical
profiles depend on horizontal Cartesian
coordinates. Initialized with any EnvironScenario

For propagation models supporting fully $3 D$ variations in the atmospheric and ground ground properties, specified in Cartesian coordinates.

A conceptual distinction is made here between the geometry of the environmental representation (e.g., homogeneous, vertical profile, or 3D, as discussed in section 6.1) and the geometry of the media representation used by the propagator. For example, a propagation algorithm may be capable of calculations with $3 \mathrm{D}$ environmental data on a Cartesian grid, but 
if only vertical profiles are available, the algorithm may still function perfectly well with this more limited data. Hence there is not a one-to-one mapping from the three environmental representations to any of the three acoustic media representations.

The AcousticMediaVert and AcousticMediaCart classes can be used in full EASEE calculations. However, the AcousticMediaPolar can be used only for standalone propagator calculations, as described at the end of section 5.1. The reason is that the polar-coordinate calculation is centered on the source position, which is not known a priori, when the propagator is constructed. To accommodate such situations, methods have been added to construct objects of the AcousticMediaPolar class by interpolating the medium representation in the AcousticMediaCart class once the source coordinates are known at runtime. Hence, propagation algorithms that naturally function in polar coordinates are supported by representing the medium in Cartesian coordinates and, then, interpolating to polar coordinates as needed.

Figure 21 shows the various acoustic propagators currently implemented in EASEE. Detailed descriptions of several of these algorithms will be provided later in this section. Most of the propagators use AcousticMediaVert or AcousticMediaCart. The PE algorithms naturally use the polar-coordinate representation in $3 \mathrm{D}$, but implementations are also available that use Cartesian coordinates and convert to polar coordinates to perform the actual calculation. Two of the acoustic propagation algorithms, namely a simple geometric spreading algorithm and the Nord2000 algorithm, use BasePropagatorMedia directly. In the former case, the algorithm was implemented in this manner because it did not require any acoustics-specific medium properties. In the latter case, BasePropagatorMedia was used directly because the Nord2000 algorithm requires spatially varying structure-function and surface roughness properties, which are not part of the acoustic media representations.

\subsubsection{Impedance-plane model}

The impedance-plane model provides an accurate calculation of the interaction of sound waves with a partially reflective ground surface. For the pertinent equations, see Salomons (2001), Ostashev and Wilson (2015), and references therein. The impedance-plane model does not include refraction by wind and temperature gradients. 
Figure 21. Acoustic propagators (propagation algorithms) implemented in EASEE, as grouped according to the medium representation upon which they are based.

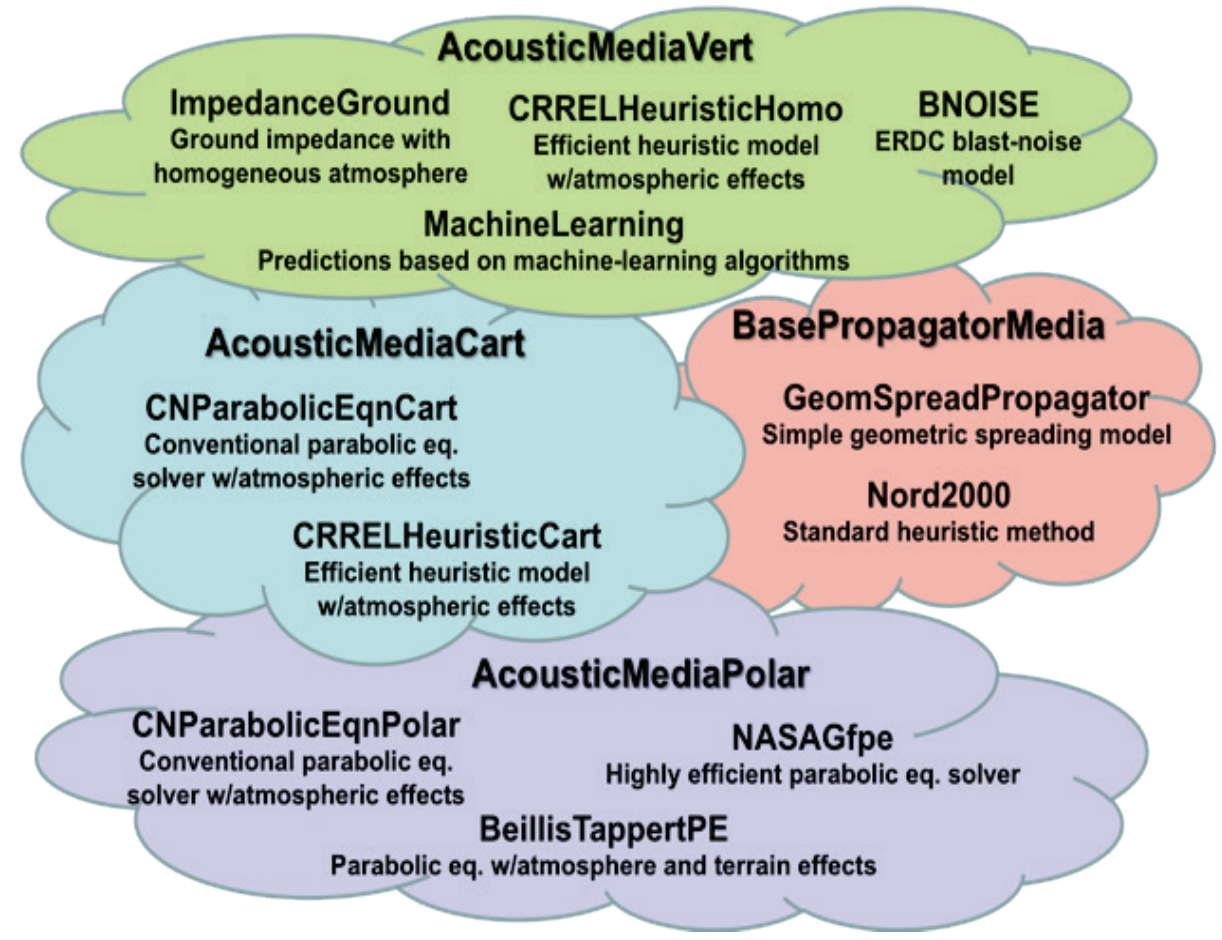

It is suitable for both low- and high-altitude sound sources. Non-flat terrain can be optionally included in a post-processing stage using EASEE's wedgediffraction model, which is provided by the BaseStructGridPropagator.

Two versions of the impedance-plane model are implemented in EASEE v3, ImpedanceGroundMediaHomo and ImpedanceGroundMediaInhomo. These implement calculations for homogeneous and inhomogeneous (spatially varying) ground-surface properties. The former uses AcousticMediaVert, whereas the latter uses AcousticMediaCart. ImpedanceGroundMediaHomo, the homogeneous ground calculation, is essentially the legacy version of the calculation from earlier versions of EASEE. ImpedanceGroundMediaInhomo, the inhomogeneous calculation, is new with EASEE v3. It uses the ground properties at the point of reflection to calculate the impedance.

\subsubsection{CRREL heuristic model}

The CRREL heuristic model is like the impedance-plane model, except that it includes a simple phenomenological adjustment for refraction in the atmosphere. The basic premise is to treat this adjustment as an additive correction in decibels, which depends on the meteorological condi- 
tions. This sort of general approach is widely used in standards (e.g., International Organization for Standardization (ISO) 1996) and other predictive models for near-ground sound propagation. In previous practice, the adjustment was typically calculated based on wind speed and surface heat flux (e.g., Marsh 1982).

However, refraction depends on the wind and temperature gradients, rather than on wind speed and surface heat flux per se. Thus, Wilson (2003) proposed characterizing the strength of refraction with an effective soundspeed scale

$$
c_{*, \mathrm{eff}}=c_{*}+u_{*} \cos \phi
$$

where $c_{*}$ is scale representing the sound-speed fluctuations, $u_{*}$ is the friction velocity, and $\phi$ is the propagation direction relative to the wind. (Also see Ostashev and Wilson 2015.)

The parameter $c_{*, \text { eff }}$ is proportional to the gradient responsible for refraction. A negative value of $c_{* \text {,eff }}$ leads to upward refraction, which typically diminishes sound levels, whereas a positive value leads to downward refraction, which typically enhances sound levels.

This discussion motivates the following heuristic formulation for a meteorological correction factor, $C_{\text {met }}$, based on $c_{*, \text { eff }}$ :

$$
C_{\text {met }}=C_{0} \min \left(1, \max \left(-1,2 c_{*, \text { eff }}\right)\right) \text {. }
$$

The calculated value for $C_{\text {met }}$ is added to the prediction from the impedance-plane model. Here, $C_{0}$ is a parameter indicating the largest absolute value for the correction. The multiplication by 2 , and the min and max functions, effectively means that the $c_{*, \text { eff }}$ values are restricted to the range $\left|c_{*, \text { eff }}\right| \geq 0.5 \mathrm{~m} / \mathrm{s}$. Values outside this range would be very unusual. Data in Marsh (1982) indicate that, for near-ground propagation, sound levels are rarely diminished by more $10 \mathrm{~dB}$ in conditions of strong upward refraction and are rarely enhanced by more than $10 \mathrm{~dB}$ in conditions of strong downward refraction. Thus, $C_{0}=10 \mathrm{~dB}$ might be regarded as reasonable. In the current implementation of the heuristic model, however, $C_{0}$ is set to $5 \mathrm{~dB}$ by default to produce a more conservative adjustment. This value can be adjusted if desired. 
As with the impedance-plane model, two versions of the CRREL heuristic model have been implemented in EASEE v3, which use AcousticMediaVert and AcousticMediaCart, for homogeneous and inhomogeneous environments, respectively. The latter version determines the friction velocity and sound-speed scale based on the coordinates of the ground reflection.

\subsubsection{BNOISE model}

The BNOISE model was originally intended for blast-noise propagation. Under the hood, BNOISE performs a table lookup according to results aggregated from fast-field program (FFP) computations. The FFP is a wavenumber integration method; that is, the calculations are performed in the spatial wavenumber domain and, then, an inverse Fourier transform is applied to convert them to the spatial domain (Salomons 2001, Ostashev and Wilson 2015). The FFP computations assume a source height of $3 \mathrm{~m}$, a receiver height of $1.5 \mathrm{~m}$, and two types of flat terrain. Desert terrain is represented by a rigid boundary, and a grass terrain uses a flow resistivity of $200 \mathrm{kPa} \mathrm{s} \mathrm{m}^{-2}$.

The FFP predictions are consolidated into propagation tables for one-third octave bands from $1 \mathrm{~Hz}$ to $20 \mathrm{kHz}$, propagation ranges from $1 \mathrm{~m}$ to $20 \mathrm{~km}$, upwind and downwind conditions, and various atmospheric stability conditions. These tables specify negative excess attenuation predictions for frequency bands, numbering from $0-43(1 \mathrm{~Hz}$ to $19,952 \mathrm{~Hz}$, using exact base-ten central frequencies), and for analogous "range bands," numbering from 0-43 ( $1 \mathrm{~m}$ to $19,952 \mathrm{~m}$ ). The frequency resolution of the original FFP calculations was $1 \mathrm{~Hz}$, stepping through frequencies from $1 \mathrm{~Hz}$ to $1000 \mathrm{~Hz}$. The range resolution was $10 \mathrm{~m}$, from $10 \mathrm{~m}$ to $10 \mathrm{~km}$. In some cases, no tabulated values exist for particular frequency and range bands, due to the spectral and range resolution of the FFP calculations. Some internal interpolation is handled for frequency bands and range bands. Plus, some extrapolation is used for long ranges and high frequencies. Across frequency gaps, linear interpolation follows a linear model in decibels against the basis of the band index. All range interpolations are treated analogously, with the exception that range indices may be any real value. Above $1 \mathrm{kHz}$ and above $10 \mathrm{~km}$, no FFP calculations were made, so extrapolations are made with constant spectra. When modeling the negative excess attenuation (NEA), any tabulated value outside the range $(-15 \mathrm{~dB}$, $+15 \mathrm{~dB}$ ) is forced back into that interval. This last step avoids excessive errors in cases where turbulent scattering becomes very significant. 
The integration of BNOISE into EASEE assumes a horizontally stratified environment, as represented by AcousticMediaVert. The BNOISEMedia class, which extends AcousticMediaVert, also includes the Pasquill-Gifford stability class, wind speed, and wind direction, as needed for the table lookup. Examples of BNOISE predictions as implemented in EASEE can be found in Glaser et al. (2017).

\subsubsection{Nord2000 model}

Nord2000 (Plovsing 2006) was originally developed in the Nordic countries for noise-control applications but has since become more widely used. It provides an accurate calculation of the interaction of sound waves with the ground surface and a well-developed terrain diffraction model. Since Nord200o involves approximations intended for sources and receivers very close to the ground, it is not suitable for aircraft at altitudes above roughly $30 \mathrm{~m}$. It is also not necessarily intended for low frequencies, which tend to be important in military applications.

For integration into EASEE, Nord2000 was packaged into a Windows dynamic link library ( ${ }^{*}$.dll file), which is run through the Java Native Interface (JNI). A Java "wrapper" class called Nord20oModel was built that invokes JNI to run Nord200O with a variety of input configurations and retrieve the predictions from file. Nord200oModel is called by Nord200oPropagatorHomo to perform a calculation with a homogeneous ground surface and atmosphere and by Nord200oPropagatorCart to perform a calculation with an inhomogeneous ground surface and atmosphere. As was discussed in section 6.5 .2 , these propagators use media classes that are built outside the normal framework for acoustical calculations, since they use certain ground and atmospheric properties (such as surface roughness and turbulence structure-function parameters) that are not used by the other acoustical calculations. Wilson et al. (2016) provides an example application of Nord200o to modeling audibility of a helicopter at low altitude.

\subsubsection{Parabolic equations}

The earliest versions of EASEE included a Crank-Nicholson (CNPE) algorithm coded in Java. The CNPE solves a parabolic (one-way) approximation to the full wave equation. It is a widely used method for wave propagation calculations that accounts for interactions with an impedance ground boundary and for refraction by wind and temperature gradients (Salomons 2001, Ostashev and Wilson 2015). The CNPE is highly accurate 
and flexible, but it is much slower than the other methods discussed thus far in this section. The calculation time and demands on computer memory increase greatly with increasing frequency. Although diffraction effects in uneven terrain can be addressed with a CNPE, the implementation in EASEE is for flat terrain. Terrain effects can be optionally included using EASEE's wedge-diffraction model, as with the impedance-plane model and other subclasses of BaseStructGridPropagator.

To address the slow calculation time characteristic of the CNPE, Gilbert and Di (1993) introduced the Green's Function Parabolic Equation (GFPE) algorithm. It solves the same PE as the CNPE but by a different numerical method involving a Fourier transform in the vertical coordinate, rather than finite differences and a tri-diagonal solver. The GFPE appears to be the fastest available algorithm that includes a rigorous calculation of refraction effects. Nonetheless, it has some drawbacks. It is somewhat less stable and flexible than the CNPE and is not well suited to calculations on uneven terrain. Because of practical limitations on the vertical computational domain size, the GFPE method is most suitable for low-altitude sources (in practice, a few hundred meters and lower). The GFPE implemented in EASEE was originally written by X. Di and K. Gilbert at the National Center for Physical Acoustics, University of Mississippi, under contract from NASA. For use in EASEE, it was compiled into a Unix executable, which is run from Windows using CygWin.

As part of EASEE v3, a third PE formulation was added, based on the Beilis-Tappert transformation (Parakkal et al. 2010). The transformation enables efficient and accurate calculations in uneven terrain. Like the CNPE, the Beilis-Tappert parabolic equation (BTPE) is coded in Java.

The PE calculations in EASEE share a common set of media classes, namely ParabolicEqMediaPolar and ParabolicEqMediaCart. As the names would suggest, they are subclasses of AcousticMediaPolar and AcousticMediaCart and support inhomogeneous calculations in polar and Cartesian coordinates, respectively. The polar versions can be initialized with an EnvironVertProf object, thus in effect enabling them to be used for homogeneous calculations with a single set of vertical profiles.

\subsubsection{Machine-learning algorithms}

EASEE v3 incorporates a novel computational approach for near-ground sound propagation, namely machine-learning algorithms (Hart et al. 
2016). Specifically, the Java class MachineLearningPropagator provides an interface that can call four different pre-trained machine-learning algorithms: an artificial neural network, bagged decision tree regression, random forest regression, and boosting regression.

The machine-learning algorithms were trained using a dataset of 27,000 CNPE calculations with randomized source frequency, source height, atmospheric parameters (wind direction, friction velocity, roughness height, sensible heat flux, boundary-layer depth), and ground parameters (static flow resistivity and ground porosity) (Hart et al. 2016). This parameterization is intended for near-ground sound propagation only. As described by Hart et al., the trained machine-learning algorithms performed very well for this scenario, with skill scores (a normalized measure of prediction accuracy) exceeding $99 \%$ for each of the algorithms. The predictions from the pre-trained algorithms are nearly instantaneous.

The machine-learning algorithms were developed using the MATLAB Statistics and Machine-Learning Toolbox. MachineLearningPropagator invokes these algorithms by calling the MATLAB code from Java. MachineLearningPropagator employs a media class called MachineLearningMediaHomo, which is an extension of AcousticMediaVert. Only calculations with homogeneous ground surfaces and a single atmospheric profile are presently supported. The atmospheric and ground parameters described above are derived from the AcousticMediaVert object.

\subsubsection{Acoustic noise backgrounds}

New acoustic noise backgrounds natural settings were added based on measurements made by Ambrose (2006) in the Grand Canyon, as part of a study funded by the National Park Service (NPS). In EASEE, these are identified as the NPS noise backgrounds. The types available are identified as Coniferous Forest, Desert Scrubland, Pinyon-Juniper, Quiet, and Water with Rapids. Figure 22 shows plots of these noise backgrounds.

In addition, acoustic noise backgrounds for city, industrial, and rural environments were added based on CRREL field studies (Albert and Decato 2017). The types available are Rural, Industrial Yard, Industrial Construction, Industrial Crusher, City Residential, City Outlying Hi Rise, City Core, and City Commercial. Figure 23 shows plots of these noise backgrounds. 
Figure 22. NPS acoustic noise backgrounds.

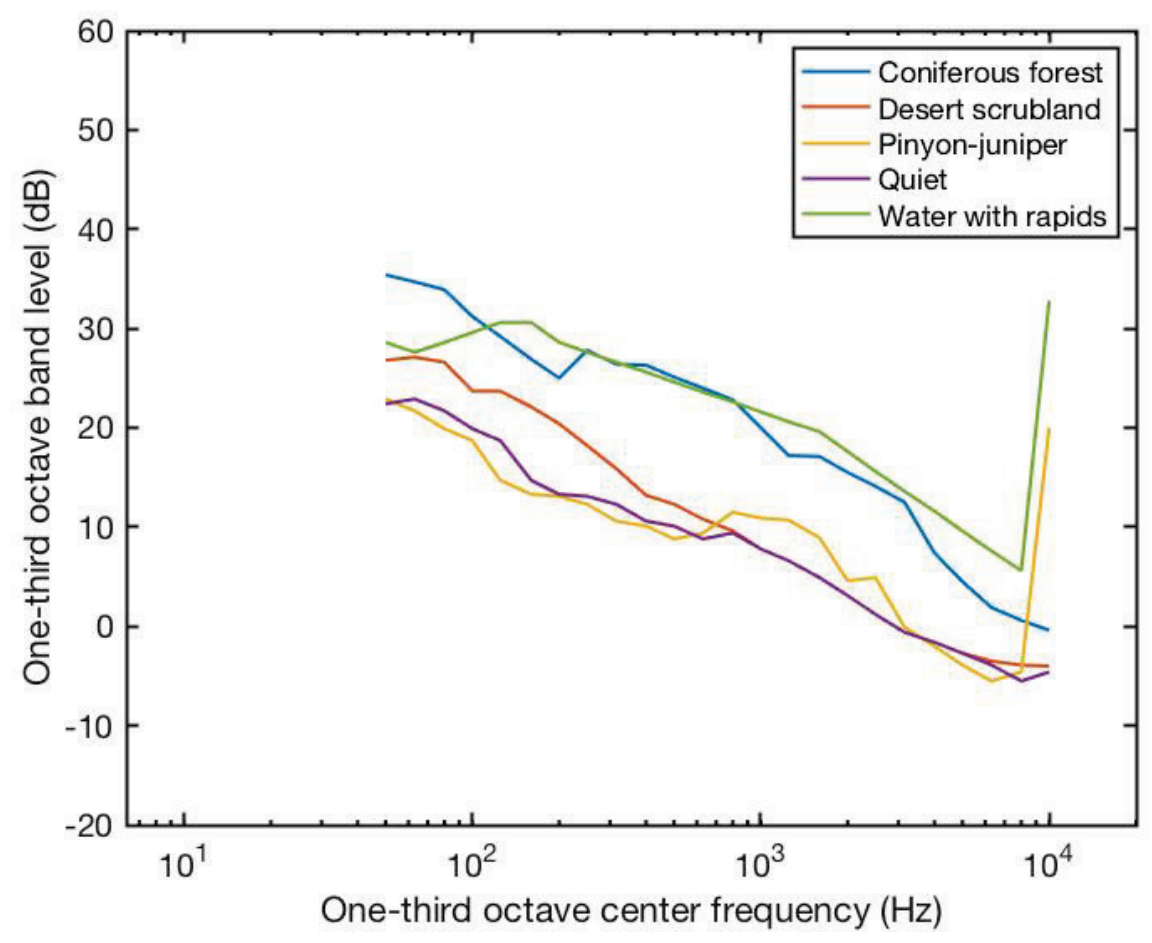

Figure 23. CRREL acoustic noise backgrounds.

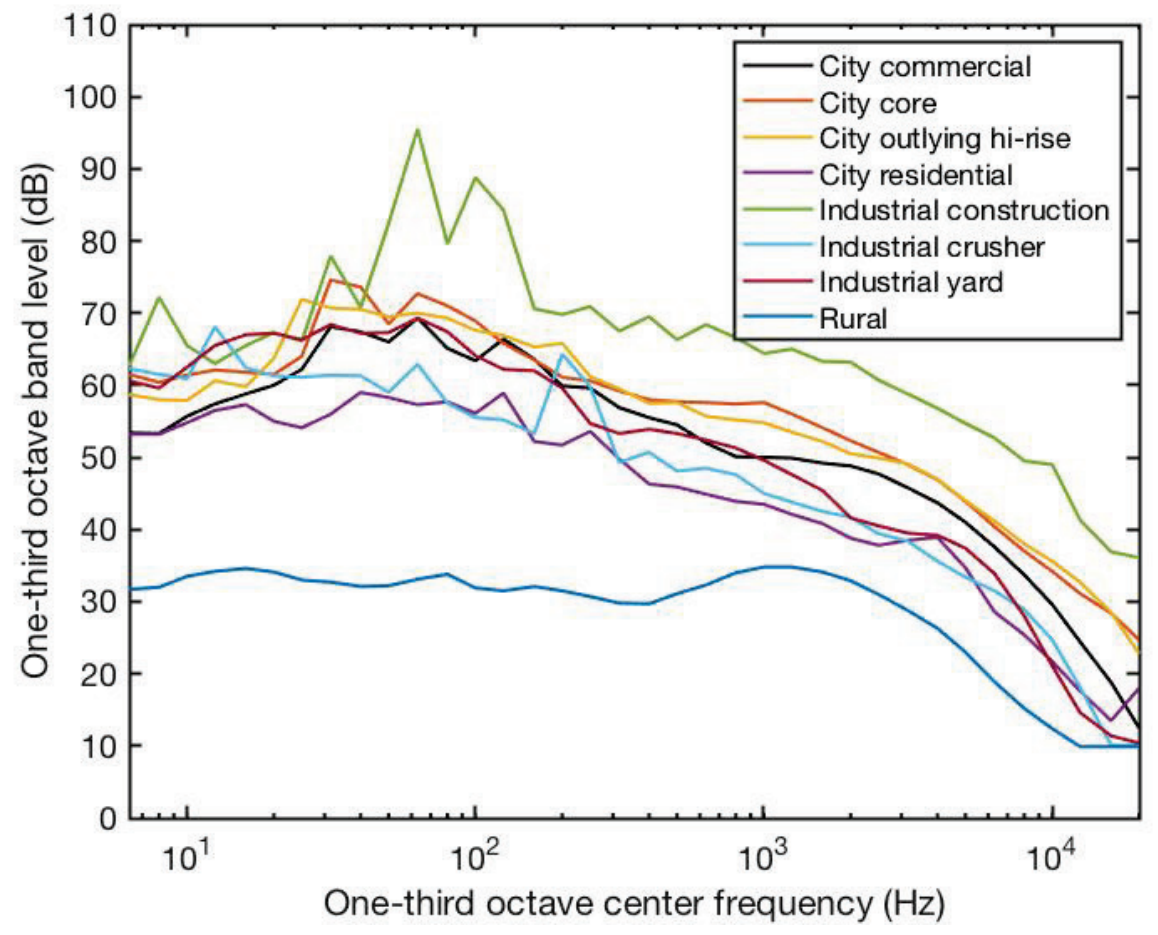




\subsection{RF modeling enhancements}

One of the primary programmatic objectives of SPRUCE was to upgrade the $\mathrm{RF}$ capabilities in EASEE. The starting point was an extensive reformulation of the RF feature definitions, which (as mentioned in section 6.5.1 with regard to acoustics) play a key role in the EASEE architecture. The environmental models (media classes) were also revamped to coincide with the structure of EASEE v3. Furthermore, propagation models and noise models were added. All these upgrades are overviewed in this section.

Transparently to the user, EASEE models the pdfs for RF signal and noise power using exponential distributions (which are equivalent to Rayleigh distributions for the amplitude). This is commonly called a strong, deep, or Rayleigh fading model in the literature. This is the same approach used for the acoustic signal modeling. Although we would prefer to implement a more general fading model, we have not yet succeeded in devising analytically or numerically tractable alternatives.

\subsubsection{Feature definitions}

Definition of RF features is made very challenging by the breadth of the RF spectrum and the physical phenomena involved in the generation, propagation, and sensing of RF signals. Before EASEE v3, the basic RF feature definitions spanned the entire electromagnetic spectrum from o Hz to 30 THz. However, since models implemented in EASEE must declare the features to which they are applicable (through the Java generics functionality described in section 1.1) and return meaningful results for these features, and since there are few if any useful modeling capabilities applicable across the entire electromagnetic spectrum, it was essentially impossible for any modeling capability to truly apply to all features in the feature space. Therefore, it was decided to subdivide the electromagnetic spectrum into smaller regions and to focus modeling efforts on the most Armyrelevant regions of the spectrum.

Most tactical and communication problems of interest to the Army occur in the VHF, UHF, and SHF regions of the electromagnetic spectrum. Thus, we focused modeling efforts on these regions. In particular, the primary frequency bands in use by the military for point-to-point communications is $30-512 \mathrm{MHz}$, which are in the VHF/UHF region. Lower frequencies are used for long-haul communications, although we are not presently en- 
deavoring to model those scenarios in EASEE. We did, however, opt to include the SHF and EHF ranges because various LOS communications and radar systems operate in this range.

For present purposes, we define the VHF as spanning frequencies from $30 \mathrm{MHz}$ to $300 \mathrm{MHz}$ (wavelengths from $10 \mathrm{~m}$ to $1 \mathrm{~m}$ ). This is the customary definition of the VHF range and is consistent with ITU (International Telecommunication Union) and IEEE (Institute of Electrical and Electronic Engineers) standards. We define the UHF range as spanning frequencies from $300 \mathrm{MHz}$ to $1 \mathrm{GHz}$ (wavelengths from $1 \mathrm{~m}$ to $30 \mathrm{~cm}$ ). This definition is consistent with the IEEE standard 521-2002, although conventional practice places the upper cut-off frequency for the UHF at $3 \mathrm{GHz}$. Our reason for adopting the IEEE definition is that many RF propagation models apply to frequencies up to a maximum of $1 \mathrm{GHz}$. Also, we wished to avoid partitioning the frequency ranges used by WLANs, which are most often around $2.4 \mathrm{GHz}$ and $5 \mathrm{GHz}$, into two separate feature sets. The SHF range is defined in EASEE as frequencies from $1 \mathrm{GHz}$ to $30 \mathrm{GHz}$ (wavelengths from $30 \mathrm{~cm}$ to $1 \mathrm{~cm}$ ), and the EHF range from $30 \mathrm{GHz}$ to $300 \mathrm{GHz}$ (wavelengths from $1 \mathrm{~cm}$ to $1 \mathrm{~mm}$ ).

The VHF and UHF ranges are combined into one feature set (family) called RFMeterSpectralFeature, whereas the SHF and EHF ranges are combined into a second called RFCentiMeterSpectralFeature. In practice, this means that EASEE maintains two separate RF modeling sequences: one for VHF and UHF, and the other for SHF and EHF. Within each of these feature families, separate models must be created for signatures, propagation, sensing, and noise backgrounds. Furthermore, the phenomena in these ranges are generally quite different. For example, in the VHF and UHF range, diffraction effects around buildings, etc., are often an important consideration for propagation modeling, whereas in the SHF and EHF range, LOS propagation can often be assumed. In the VHF and UHF range, standardized models for the noise background are often available. In the SHF and EHF range, the noise floor of the sensor is often the limiting factor in sensor performance. Figure 24 shows the Java classes defining the RF feature sets and their implementations. 


\subsubsection{RF media and propagation models}

At present, the selection of RF models is not nearly as extensive as the acoustic models. Figure 25 shows the current and planned suite of RF signal propagation models, grouped according to the media (environmental) representations upon which they are based.

Figure 24. RF feature classes in EASEE. Several feature sets (families) are defined through Java interfaces. Two of these, namely RFCentiMeterSpectralFeature (for the SHF/EHF frequency range) and RFMeterSpectralFeature (for the VHF/UHF frequency range) have been developed extensively, including Java enumeration classes to define specific features.

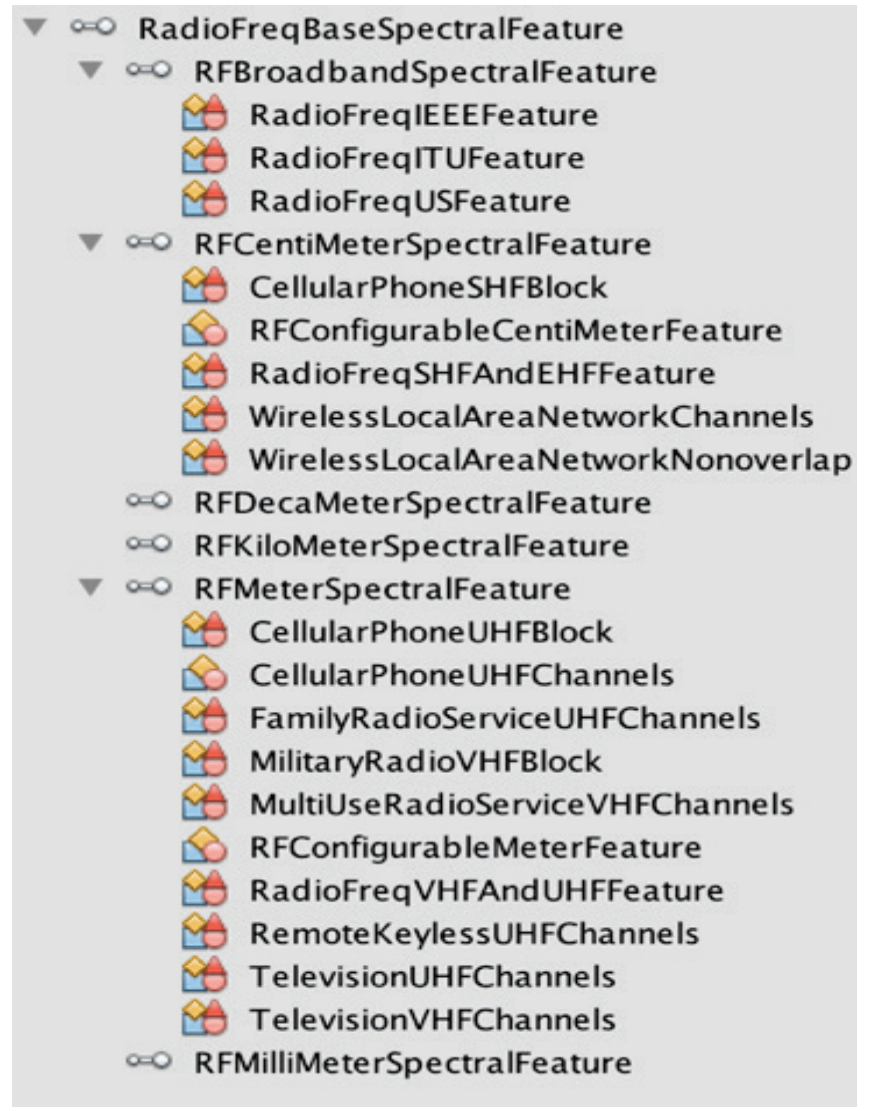


Figure 25. RF propagators (propagation algorithms) implemented in EASEE, as grouped according to the medium representation upon which they are based.

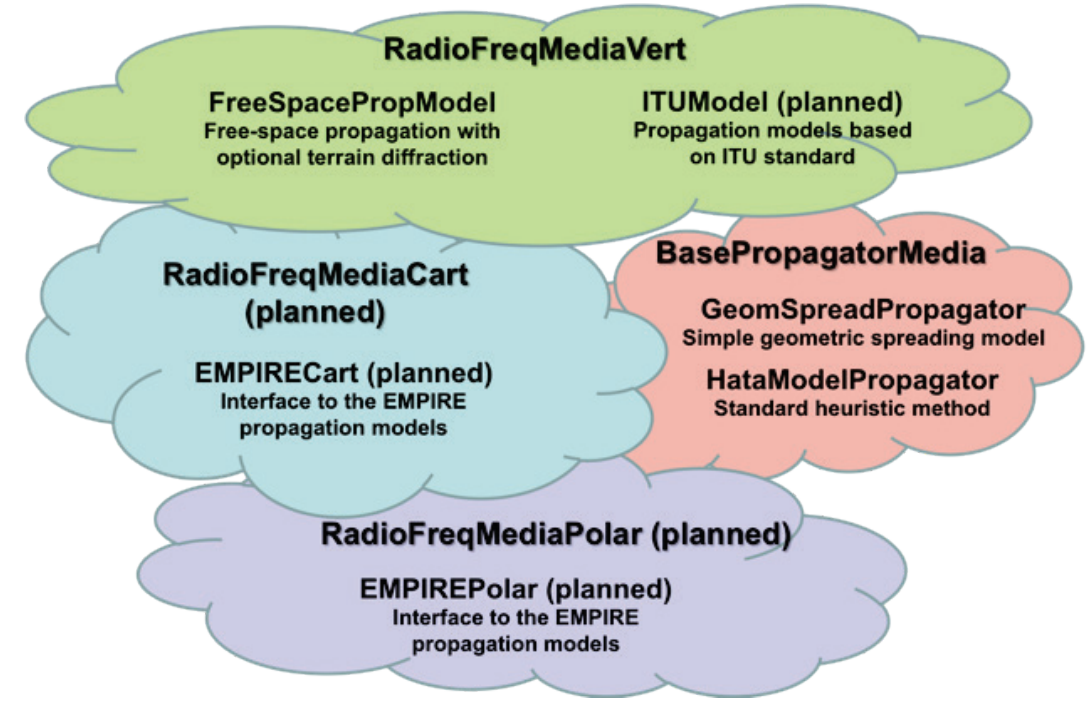

Some of the general-purpose basic propagation models in EASEE may be used for RF modeling. These include GeomSpreadPropagator, which accommodates arbitrary geometric spreading losses, including cylindrical $(1 / R)$ and spherical $\left(1 / R^{2}\right)$. (Here $R$ is the distance between the source and receiver.) The propagation may take place in free space, or over a perfectly reflecting boundary with an amplitude reflection coefficient of +1 (for a reflection perfectly in phase with the incident wave) or -1 (for a reflection perfectly out of phase with the incident wave). This is sometimes called a two-ray or image-source model. An exponential attenuation factor, describing the diminishment in signal power with distance along the direct and reflected paths, may also be included.

Another potentially useful model is the LOS propagator (LineOfSightPropagator), which implements a LOS calculation over terrain of varying elevation. Geometric spreading and attenuation can also be included in the LOS calculation, as can a finite field-of-view. The PLOS calculation (ProbLineOfSightPropagator) described in section 6.3 may also be used for RF modeling. The LOS and PLOS calculations are potentially particularly useful for the SHF and EHF frequency range (the RFCentiMeterSpectralFeature feature family), since the wavelengths are small enough in that region that LOS calculations are typically reasonable.

At the present time, two propagators specific to RF propagation modeling have been implemented: the "free space" and Hata model propagators. The free space propagation model (FreeSpacePropModel), which applies to the 
RFMeterSpectralFeature feature family, is very similar to GeomSpreadPropagator, in that it incorporates geometric spreading loss and reflections. But, it has the additional advantage of being an extension of the BaseStructGridPropagator class, which (despite the somewhat misleading name) means that it incorporates optional terrain diffraction effects and provides efficient methods for performing radially symmetric calculations with storage of results to temporary files. The FreeSpacePropModel classes uses FreeSpacePropMedia as its medium model, which extends RadioFreqMediaVert. The latter includes a landcover specification and atmospheric profiles, which could be leveraged in the developing of future RF modeling capabilities.

The Hata (1980) model is widely used for modeling cellular transmissions in outdoor environments. The implementation in EASEE includes the COST (COopération européenne dans le domaine de la recherche Scientifique et Technique) 231 extensions, as described in Wikipedia (2019c). The Hata model is intended for frequencies between $150 \mathrm{MHz}$ and $2000 \mathrm{MHz}$, distances up to $20 \mathrm{~km}$, source heights from 30-200 m, and receiver heights from $1 \mathrm{~m}$ to $10 \mathrm{~m}$. For frequencies between $150 \mathrm{MHz}$ and $1500 \mathrm{MHz}$, the basic Hata model is applied. The COST extension is for frequencies between $1500 \mathrm{MHz}$ and $2000 \mathrm{MHz}$. The Hata model describes path loss $(L)$ in $\mathrm{dB}$ with an equation of the following form:

$$
L=B L-C F-A
$$

where $B L$ is the base loss (dependent upon frequency, distance, and transmitter height) in an urban environment, $C F$ is a correction for suburban or open environments, and $A$ is the receiver antenna correction factor, which depends on the receiver height and frequency. We refer the reader to the preceding references for the full functional forms of these terms. Different values are used depending on the environment, for which four classes are defined: large city, small/medium city, suburban, or rural.

The Java class HataModelPropagator is implemented directly as an extension of the BasePropagator class, since it does not leverage capabilities from any other propagator classes. HataModelPropagator applies only to the RFMeterSpectralFeature feature family only. The Hata model environment is determined through mapping the landcover class; for example, the "unoccupied" and "rural" landcovers are mapped to rural, "suburban" is mapped to suburban, "small city" is mapped to small and medium city, "medium city" is mapped to small and medium city, and "large city" is mapped to "large city." 


\subsubsection{RF noise backgrounds}

RF noise backgrounds, in the VHF and UHF frequency ranges, were also added based on studies in the published literature. Noise models from the literature are generally focused on unintentional emissions from manmade sources and are not intended to predict the undesired power reaching the receiver from an intentional emitter (broadcast radio, jamming systems, etc.).

In general, RF noise is assumed to have amplitudes that are circular complex Gaussian in nature, to have a power that increases with decreasing frequency, and to be generated from manmade sources. Several models for manmade noise levels as a function of frequency have been proposed, and the best-known model in the frequency range of interest is the model of Hagn and Sailors (1979). This model, combined with other data, became the basis for the ITU (2013) Recommendation 372 on Radio Noise that is the current international standard.

The ITU standard breaks the noise environment into four categories (quiet rural, rural, suburban, and city) and uses the following equation to model the median antenna noise figure $F_{a m}$, expressed in decibels above thermal noise for the bandwidth of interest $b(\mathrm{~Hz})$, as a function of frequency $f(\mathrm{MHz})$ :

$$
F_{a m}=c-d \log _{10} f
$$

The constants $c$ and $d$ are chosen based on the four environmental categories and whether the frequency of interest lies within the VHF or UHF frequency ranges (Table 1). The effect of these constants for differing noise environments and their frequency dependence are shown in Figure 26(a).

Table 1. Constants for the ITU standard and CRREL 2014 manmade noise models. VHF constants valid between 30 and $200 \mathrm{MHz}$; UHF constants valid between 200 and $1000 \mathrm{MHz}$.

\begin{tabular}{|l|l|c|c|c|c|}
\hline Model & Environment & $\boldsymbol{c}_{\boldsymbol{V H F}}$ & $\boldsymbol{d}_{\boldsymbol{V} \boldsymbol{H} \boldsymbol{F}}$ & $\boldsymbol{c}_{\boldsymbol{U} \boldsymbol{H} \boldsymbol{F}}$ & $\boldsymbol{d}_{\boldsymbol{U} \boldsymbol{F} \boldsymbol{F}}$ \\
\hline ITU & City & 76.8 & 27.7 & 49.4 & 15.8 \\
\hline & Suburb & 72.5 & 27.7 & 45.2 & 15.8 \\
\hline & Rural & 67.2 & 27.7 & 39.9 & 15.8 \\
\hline & Quiet Rural & 53.6 & 28.6 & 0 & 0 \\
\hline CRREL 2014 & City & 112.0 & 36.2 & 112.0 & 36.2 \\
\hline & Suburb & 105.0 & 36.2 & 105.0 & 36.2 \\
\hline & Rural & 90.5 & 33.0 & 140.0 & 54.7 \\
\hline & Quiet Rural & 61.5 & 20.4 & 140.0 & 54.7 \\
\hline
\end{tabular}


Experimental studies from the late 1990 s conducted in the United States found that the data and the ITU recommendation 372 agreed within one standard deviation in the VHF and UHF bands. However, Wagstaff and Merricks (2005) found that the noise background levels are significantly higher than those predicted by the ITU model.

While Wagstaff and Merricks did not explicitly determine the $c$ and $d$ constants from their data to fit the ITU model, we were able to analyze and extract the necessary values from their published figure. These extracted constants were then used to establish CRREL 2014 values, plotted in Figure 26(b). The CRREL 2014 values for UHF and VHF represent a compromise between past and current understanding; it may also be more representative of regions with population densities greater than 100 persons per square $\mathrm{km}$.

Figure 26. Plots of $\boldsymbol{F}_{\boldsymbol{a}}$ as a function of frequency for (a) the standard ITU + Hagn \& Sailors (1979), and (b) the CRREL 2014 noise model, based on the work of Wagstaff and Merricks (2005). The change in slope at $200 \mathrm{MHz}$ results from the changeover from VHF to UHF noise constants.
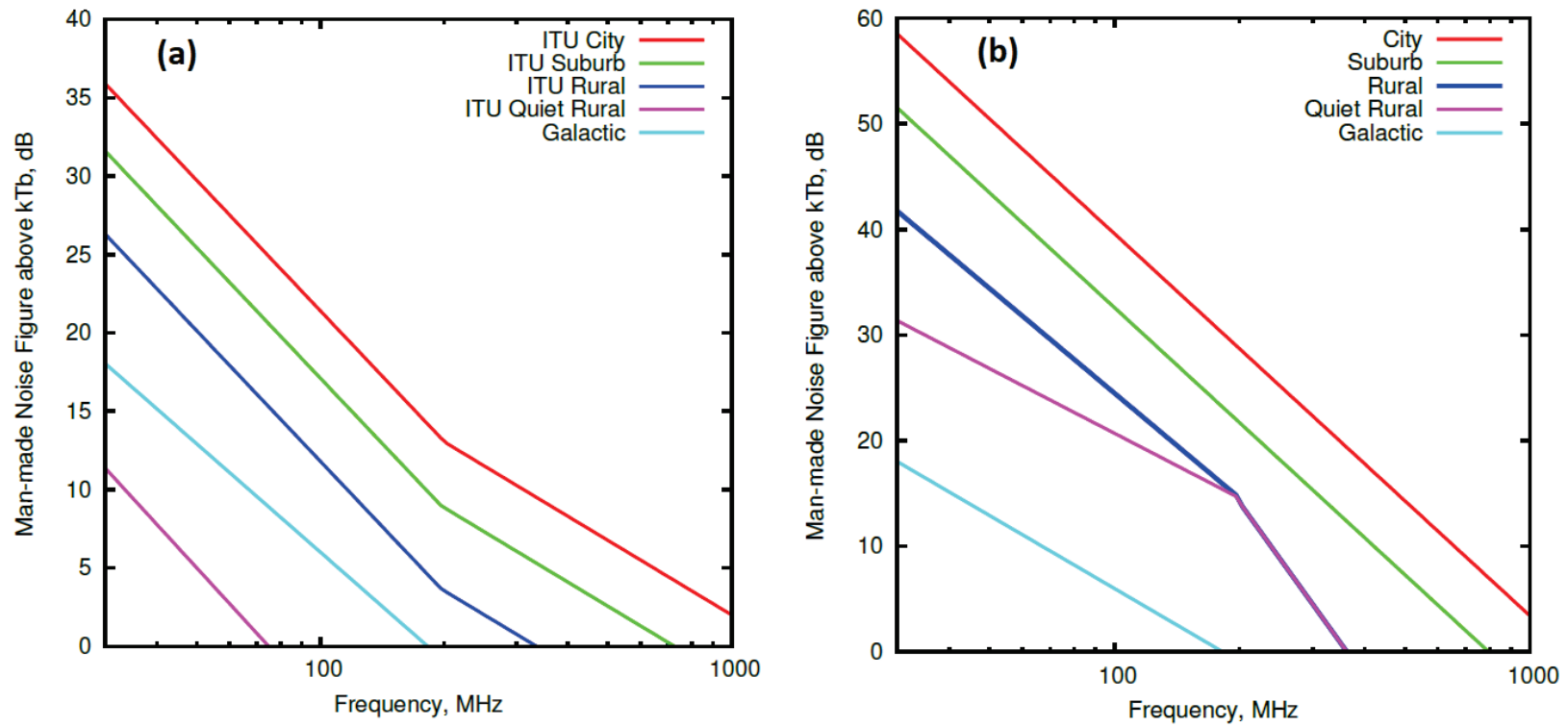

Information on RF noise background in the SHF and EHF frequency range is essentially non-existent in the literature because systems operating in this range are typically limited by internal receiver noise. Unless site-specific information is available on ambient noise, it is best to analyze system performance based on internal noise specifications. However, site-specific noise data are available based on data recorded by CRREL scientists at the Quantico Urban Training Center in Virginia. These noise levels are available within EASEE's RF noise model and are designated "Marine Corps 
Base Quantico, quiet” and "Marine Corps Base Quantico, noisy." Since background noise modeling in this frequency range is a relatively new endeavor and very sensitive to the electronic noise in the measurement system, calculations based on these backgrounds should be regarded as having a high degree of uncertainty. 


\section{Conclusion}

This report described extensions to the EASEE software to create a suite of $3 \mathrm{D}$ signal and sensor performance modeling capabilities that realistically capture propagation physics in urban, mountainous, forested, and other complex terrain environments. In such environments, inhomogeneous terrain and $3 \mathrm{D}$ weather have significant impacts on the propagation. Addressing this problem in an accurate and efficient manner required extensive attention to details of how the environment is represented and converted to information needed by the propagation models. The approach described here uses a fully independent environmental data layer (abstraction layer) that ingests data from a great variety of terrain and atmospheric data resources in a highly flexible manner. The process of translating the environmental data into information needed for acoustic, seismic, RF, and optical signal propagation modeling involved very complex software design techniques based on object orientation and Java generics. The result is a general, robust software framework enabling advanced signal modeling capabilities on a desktop or laptop computer, which is called EASEE v3.

Some other important enhancements with EASEE v3 include modeling of transmitter and receiver directivity functions in $3 \mathrm{D}$, including rotations of the transmitter and receiver platforms; an Extensible Markup Language/JavaScript Object Notation (XML/JSON) interface to facilitate deployment of web services; support for arbitrary geographic coordinate projections; probabilistic calculations for LOS in complex terrain and vegetation; and new signal feature definitions and other support for infrasound and RF modeling. In particular, extensive modeling capabilities for RF modeling in the VHF and UHF and SHF and EHF frequency ranges were added, including transmitter models, the Hata propagation model, receiver models, and noise backgrounds.

With version 3, EASEE made the leap from an architecture predominantly based on horizontally stratified environments (vertical atmospheric and seismic structure only) to one supporting fully $3 \mathrm{D}$ environments. To capitalize on this foundation, additional innovative algorithms and numerical methods capable of realistically and efficiently handling multiple scattering and other complexities of urban and rugged terrain environments will need to be devised and implemented. Doing so will help open the door to important new and timely applications, such as Arctic domain awareness, defense against unattended aerial systems and missiles, signature control, 
sensor system design and acquisition, and operations in urban and densely forested terrain against near-peer adversaries.

In the future, we also envision employing EASEE as part of context-aware systems. Previously, EASEE has been employed in conventional GUIs that implicitly assume the user has some training and time to interact with the software in a deliberate way, e.g., by choosing the desired AOI, targets, sensors, and environmental conditions. When Army units are directly engaging hostile forces, however, the software will need to automatically anticipate the sensor performance and vulnerability analyses needed to successfully perform an operation, gather the environmental and tactical data for the predictions, and proactively run and display products of high relevance to the user. The creation of such context-aware systems is anticipated to be a primary technical focus for future EASEE-related research. 


\section{References}

Albert, D. G., and S. N. Decato. 2017. "Acoustic and Seismic Ambient Noise Measurements in Urban and Rural Areas.” Applied Acoustics 119:135-143.

Ambrose, S. 2006. Sound Levels in the Primary Vegetation Types in Grand Canyon National Park, July 2005. National Park Service Report No. GRCA-05-02. https://www.nps.gov/grca/learn/nature/upload/ambient-sound.pdf.

Bullington, K. 1977. "Radio Propagation for Vehicular Communications." IEEE Transactions on Vehicular Technology 26:295-308.

Fidell, S., K. S. Pearsons, and R. L. Bennett. 1972. Predicting Aural Detectability of Aircraft in Noise Backgrounds. AFFDL-TR-72-17. Wright-Patterson Air Force Base, OH: Air Force Flight Dynamics Laboratory.

Frankenstein, S., and G. G. Koenig. 2004. Fast All-Season Soil Strength (FASST). ERDC/CRREL SR-04-1. Hanover, NH: U.S. Army Engineer Research and Development Center, Cold Regions Research and Engineering Laboratory (ERDC-CRREL).

Gilbert, K. E., and X. Di. 1993. “A Fast Green's Function Method for One-Way Sound Propagation in the Atmosphere." Journal of the Acoustical Society of America 94:2343-2352.

Glasberg, B. R., and B. C. J. Moore. 1990. "Derivation of Auditory Filter Shapes from Notched-Noise Data.” Hearing Research 47:103-138.

Glaser, D. R., D. K. Wilson, L. E. Waldrop, C. R. Hart, M. J. White, E. T. Nykaza, and M. E. Swearingen. 2017. "Modeling of Signal Propagation and Sensor Performance for Infrasound and Blast Noise." In Proceedings Volume 1019o, Ground/Air Multisensor Interoperability, Integration, and Networking for Persistent ISR VIII. Anaheim, CA: SPIE Defense and Commercial Sensing. https://doi.org/10.1117/12.2262683.

Hagn, G. H., and D. B. Sailors. 1979 "Empirical Models for Probability Distributions of Short-term Near Environmental Manmade Radio Noise Levels.” Electromagnetic Compatibility, 1979: 3rd Symposium and Technical Exhibition on Electromagnetic Compatibility, Rotterdam May 1-3, 1979. p 355.

Hart, C. R., N. J. Reznicek, D. K. Wilson, C. L. Pettit, and E. T. Nykaza. 2016. "Comparisons Between Physics-Based, Engineering, and Statistical Learning Models for Outdoor Sound Propagation." Journal of the Acoustical Society of America 139(5):2640-2655.

Hata, M. 1980. "Empirical Formula for Propagation Loss in Land Mobile Radio Services" IEEE Transactions on Vehicular Technology 29(3):317-325.

ISO (International Organization for Standardization). 1996. ISO 9613-2, Acoustics Attenuation of Sound during Propagation Outdoors - Part 2: General Method of Calculation. Geneva, Switzerland: ISO. 
ITU (International Telecommunication Union). 2013. Radio Noise, Recommendation P.372-11. Geneva, Switzerland: ITU.

Marsh, K. J. 1982. "The CONCAWE Model for Calculating the Propagation of Noise from Open-Air Industrial Plants." Applied Acoustics 15(6):411-428.

Ostashev, V. E., and D. K. Wilson. 2015. Acoustics in Moving Inhomogeneous Media. $2 \mathrm{~d}$ ed. Boca Raton, FL: CRC Press.

Page, J. A., C. Wilmer, and K. J. Plotkin. 2008. Rotorcraft Noise Model Technical Reference and User Manual. Version 7.1. Wyle WR 08-04. Arlington, VA: Wyle Laboratories, Inc.

Parakkal, S., K. E. Gilbert, X. Di, and H. E. Bass. 2010. "A Generalized Polar-Coordinate Method for Sound Propagation over Large-Scale Irregular Terrain.” Journal of the Acoustical Society of America 128:2573-2580.

Plovsing, B. 2006. Nord20oo. Validation of the Propagation Model. Delta Acoustics AV 1117/o6. Hørsholm, Denmark: Danish Electronics, Light \& Acoustics.

Salomons, E. M. 2001. Computational Atmospheric Acoustics. Dordrecht, The Netherlands: Kluwer Academic Publishers.

Vecherin, S. N., D. K. Wilson, and V. E. Ostashev. 2011. "Incorporating Source Directionality into Outdoor Sound Propagation Calculations." Journal of the Acoustical Society of America 130(6):3608-3622.

Wagstaff, A., and N. Merricks. 2005. “Manmade Noise Measurement Programme.” IEEE Proceedings-Communications 152(3):371-377.

Waldrop, L. E., D. K. Wilson, M. T. Ekegren, and C. T. Borden. 2017. "EASEE: an Open Architecture Approach for Modeling Battlespace Signal and Sensor Phenomenology.” Anaheim, CA: SPIE Defense and Commercial Sensing.

Wikipedia. 2019a. "Euler Angles." Wikipedia, The Free Encyclopedia. https://en.wikipedia.org/w/index.php?title=Euler angles\&oldid=870332149.

Wikipedia. 2019b. "Aircraft Principal Axes." Wikipedia, The Free Encyclopedia. https://en.wikipedia.org/w/index.php?title=Aircraft principal axes\&oldid=868 124208.

Wikipedia. 2019c. "COST Hata Model." Wikipedia, The Free Encyclopedia. https://en.wikipedia.org/w/index.php?title=COST_Hata_model\&oldid=8611575 74 .

Wilson, D. K. 1998. A Prototype Acoustic Battlefield Decision Aid Incorporating Atmospheric Effects and Arbitrary Sensor Layouts. ARL-TR-1708. Adelphi, MD: U.S. Army Research Laboratory.

Wilson, D. K. 2003. "The Sound-Speed Gradient and Refraction in the near-Ground Atmosphere." Journal of the Acoustical Society of America 113(2): pp.750-757. 
Wilson, D. K., R. Bates, and K. K. Yamamoto. 2009. Object-Oriented Software Model for Battlefield Signal Transmission and Sensing. ERDC/CRREL TR-09-17. Hanover, NH: ERDC-CRREL.

Wilson, D. K., and K. K. Yamamoto. 2014. Environmental Awareness for Sensor and Emitter Employment (EASEE): Software Design Version 2. ERDC/CRREL TR14-27. Hanover, NH: ERDC-CRREL.

Wilson, D. K., B. Ikelheimer, D. Conner, and J. Stephenson. 2016. "Aircraft Acoustic Detection Modeling in EASEE.” Military Sensing Symposium Specialty Group on Battlespace Acoustic, Seismic, Magnetic, and Electric-Field Sensing and Signatures (Gaithersburg, MD).

Wilson, D. K., D. J. Breton, R. E. Alter, L. E. Waldrop, W. M. Barnes, M. B. Muhlestein, and V. E. Ostashev. 2018. "Modeling RF and Acoustic Signal Propagation in Complex Environments." SPIE Defense and Commercial Sensing, Orlando, FL. 


\section{Acronyms and Abbreviations}

\begin{tabular}{|c|c|}
\hline Term & Definition \\
\hline 1D & One -Dimensional \\
\hline 2D & Two -Dimensional \\
\hline 3D & Three-Dimensional \\
\hline AFFDL & Air Force Flight Dynamics Laboratory \\
\hline ANSI & American National Standards Institute \\
\hline $\mathrm{AOI}$ & Area of Interest \\
\hline APET & (NASA) Acoustic Propagation and Emulation Toolset \\
\hline API & Application Programming Interface \\
\hline ARL & U.S. Army Research Laboratory \\
\hline ARTEMIS & Army Terrestrial-Environmental Modeling and Intelligence System \\
\hline BL & Base Loss \\
\hline BTPE & Beilis-Tappert Parabolic Equation \\
\hline CAC & Common Access Card \\
\hline CE & (Army) Computing Environment \\
\hline CERL & Construction Engineering Research Laboratory \\
\hline CNPE & Crank-Nicholson Parabolic Equation \\
\hline COAMPS & Coupled Ocean/Atmosphere Mesoscale Prediction System \\
\hline COE & (Army) Common Operating Environment \\
\hline CONCAWE & CONservation of Clean Air and Water in Europe \\
\hline COST & $\begin{array}{l}\text { COopération européenne dans le domaine de la recherche Sci- } \\
\text { entifique et Technique }\end{array}$ \\
\hline CP CE & (Army) Command Post Computing Environment \\
\hline CRREL & Cold Regions Research and Engineering Laboratory \\
\hline DEM & Digital Elevation Map \\
\hline DISA & Defense Information Systems Agency \\
\hline DoD & U.S. Department of Defense \\
\hline DREN & Defense Research and Engineering Network \\
\hline DSM & Digital Surface Model \\
\hline DTM & Digital Terrain Model \\
\hline EASEE & Environmental Awareness for Sensor and Emitter Employment \\
\hline EHF & Extremely High Frequency \\
\hline EMPIRE & $\begin{array}{l}\text { (U.S. Navy) Electromagnetic Propagation Integrated Resource } \\
\text { Environment }\end{array}$ \\
\hline ERB & Equivalent Rectangular Bandwidth \\
\hline ERDC & U.S. Army Engineer Research and Development Center \\
\hline ERDC-CRREL & $\begin{array}{l}\text { Engineer Research and Development Center, Cold Regions Research } \\
\text { and Engineering Laboratory }\end{array}$ \\
\hline EWS & EASEE Web Services \\
\hline FASST & Fast All-season Soil STrength \\
\hline
\end{tabular}




\begin{tabular}{|c|c|}
\hline Term & Definition \\
\hline FFP & Fast-Field Program \\
\hline GALWEM & Global Air-Land Weather Exploitation Model \\
\hline GFPE & Green's Function Parabolic Equation \\
\hline GIS & Geographic Information System \\
\hline GOTS & Government-owned Off-The-Shelf \\
\hline GRCA & Grand Canyon National Park \\
\hline GRE & Geospatial Research Engineering \\
\hline GUI & Graphical User Interface \\
\hline HTML & Hypertext Markup Language \\
\hline HTTP & hypertext transfer protocol \\
\hline IEEE & Institute of Electrical and Electronics Engineers \\
\hline IR & Infrared \\
\hline ISO & International Organization for Standardization \\
\hline ITL & Information Technology Laboratory \\
\hline ITU & International Telecommunication Union \\
\hline JAR & Java ARchive \\
\hline JAXB & Java Architecture for XML Binding \\
\hline $\mathrm{JNI}$ & Java Native Interface \\
\hline JSON & JavaScript Object Notation \\
\hline KML & Keyhole Markup Language \\
\hline LC & Landcover \\
\hline LiDAR & Light Detection and Ranging \\
\hline LOS & Line-of-Site \\
\hline MODTRAN & Moderate Resolution Atmospheric Transmission \\
\hline MOSA & Modular Open System Architecture \\
\hline MUSES & Multi-Service Electro-optic Signature \\
\hline NASA & National Aeronautics and Space Administration \\
\hline NEA & Negative Excess Attenuation \\
\hline NetCDF & Network Common Data Format \\
\hline NLCD & National Land Cover Database \\
\hline NPS & National Park Service \\
\hline NSN & National Supply Number \\
\hline OGC & Open Geospatial Consortium \\
\hline OMB & Office of Management and Budget \\
\hline PDF & Probability Density Function \\
\hline PE & Parabolic Equation \\
\hline PLOS & Probabilistic Line-of-Sight \\
\hline radpat & radiation pattern \\
\hline RESTful & Representational State Transfer \\
\hline $\mathrm{RF}$ & Radio Frequency \\
\hline SAR & Same As Report \\
\hline
\end{tabular}




$\begin{array}{ll}\text { Term } & \text { Definition } \\ \text { SF } & \text { Standard Form } \\ \text { SHF } & \text { Super-High Frequency } \\ \text { SOAP } & \text { Simple Object Access Protocol } \\ \text { SPIE } & \text { Security Professionals Information Exchange } \\ \text { SPRUCE } & \text { Signal Physics Representation in Uncertain and Complex Environments } \\ \text { SR } & \text { Special Report } \\ \text { SSGF } & \text { Standard Shareable Geospatial Foundation } \\ \text { SVN } & \text { Subversion } \\ \text { TR } & \text { Technical Report } \\ \text { UGS } & \text { Unattended Ground Sensors } \\ \text { UHF } & \text { Ultra-High Frequency } \\ \text { UI } & \text { User Interface } \\ \text { URL } & \text { Universal Resource Locator } \\ \text { USCS } & \text { Unified Soils Classification System } \\ \text { UTM } & \text { Universal Transverse Mercator } \\ \text { VCS } & \text { Version Control System } \\ \text { VHF } & \text { Very High Frequency } \\ \text { WGS } & \text { World Geodetic System } \\ \text { WLAN } & \text { Wireless Local Area Network } \\ \text { WRF } & \text { Weather Research and Forecasting } \\ \text { WSDL } & \text { Web Service Definition Language } \\ \text { XML } & \text { Extensible Markup Language } \\ \text { XSD } & \text { XML Schema Definition }\end{array}$




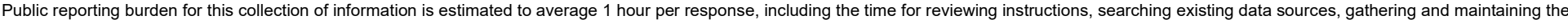

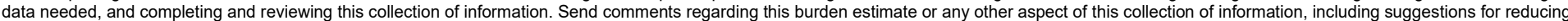

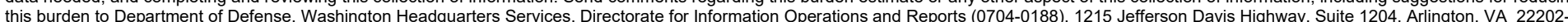

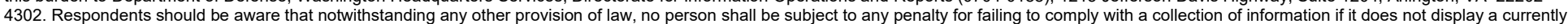
valid OMB control number. PLEASE DO NOT RETURN YOUR FORM TO THE ABOVE ADDRESS.

\begin{tabular}{c|c}
\hline 1. REPORT DATE (DD-MM-YYYY) & 2. REPORT TYPE \\
April 2021 & Technical Report (TR)
\end{tabular}

\section{TITLE AND SUBTITLE} Technical Report (TR)

Signal Propagation Modeling in Complex, Three-Dimensional Environments

3. DATES COVERED (From - To)

5a. CONTRACT NUMBER

5b. GRANT NUMBER

5c. PROGRAM ELEMENT

053HJo

\section{AUTHOR(S)}

D. Keith Wilson, Daniel J. Breton, Lauren E. Waldrop, Danney R. Glaser, Ross E. Alter, Carl R. Hart, Wesley M. Barnes, Michael T. Ekegren, Michael B. Muhlestein, Manisha Mishra, M. Andrew Niccolai, Michael J. White, Christian Borden, and Ethan Fahy

\section{5d. PROJECT NUMBER}

$\mathrm{T} 42$

5e. TASK NUMBER

5f. WORK UNIT NUMBER

\section{PERFORMING ORGANIZATION NAME(S) AND ADDRESS(ES)}

U.S. Army Engineer Research and Development Center (ERDC)

Cold Regions Research and Engineering Laboratory (CRREL)

72 Lyme Road,

Hanover, NH 03755-1290

Atmospheric and Environmental Research, Inc

Construction Engineering Research Laboratory (CERL) 2902 Newmark Drive Champaign, IL 61822

\section{PERFORMING ORGANIZATION REPORT} NUMBER

ERDC TR-21-6

131 Hartwell Avenue \#4

Lexington, MA 02421

\section{SPONSORING / MONITORING AGENCY NAME(S) AND ADDRESS(ES)}

Assistant Secretary of the Army for Acquisitions, Logistics, and Technology 103 Army Pentagon

Washington, DC 20314-1000

\section{ASA(ALT)}

11. SPONSOR/MONITOR'S REPORT NUMBER(S)

\section{DISTRIBUTION / AVAILABILITY STATEMENT}

Approved for public release; distribution unlimited.

\section{SUPPLEMENTARY NOTES}

ERDC 6.2 Geospatial Research and Engineering (GRE) ARTEMIS STO-R, funding account number U4357509

\section{ABSTRACT}

The Signal Physics Representation in Uncertain and Complex Environments (SPRUCE) work unit, part of the U.S. Army Engineer Research and Development Center (ERDC) Army Terrestrial-Environmental Modeling and Intelligence System (ARTEMIS) work package, focused on the creation of a suite of 3D signal and sensor performance modeling capabilities that realistically capture propagation physics in urban, mountainous, forested, and other complex terrain environments. This report describes many of the developed technical capabilities. Particular highlights are (1) creation of a Java environmental data abstraction layer for $3 \mathrm{D}$ representation of the atmosphere and inhomogeneous terrain that ingests data from many common weather forecast models and terrain data formats, (2) extensions to the Environmental Awareness for Sensor and Emitter Employment (EASEE) software to enable 3D signal propagation modeling, (3) modeling of transmitter and receiver directivity functions in 3D including rotations of the transmitter and receiver platforms, (4) an Extensible Markup Language/JavaScript Object Notation (XML/JSON) interface to facilitate deployment of web services, (5) signal feature definitions and other support for infrasound modeling and for radio-frequency (RF) modeling in the very high frequency (VHF), ultra-high frequency (UHF), and super-high frequency (SHF) frequency ranges, and (6) probabilistic calculations for line-of-sight in complex terrain and vegetation.

\section{SUBJECT TERMS}

Signal processing Radio frequency
Signal processing - Computer programs

Terrain analysis (Military science)--Mathematical models

\section{SECURITY CLASSIFICATION OF:}

\section{a. REPORT}

Unclassified

\section{b. ABSTRACT \\ Unclassified}

\section{c. THIS PAGE \\ Unclassified}

\section{LIMITATION OF ABSTRACT}

SAR
Radio waves

18. NUMBER OF PAGES

81 19a. NAME OF RESPONSIBLE PERSON

19b. TELEPHONE NUMBER (include area code) 